\title{
INVOLUTIVE TABLEAUX, CHARACTERISTIC VARIETIES, AND RANK-ONE VARIETIES IN THE GEOMETRIC STUDY OF PDEs
}

\author{
ABRAHAM D. SMITH \\ Department of Mathematics, Statistics and Computer Science \\ University of Wisconsin-Stout \\ Menomonie, Wisconsin 54751-2506, USA \\ E-mail:smithabr@uwstout.edu
}

\begin{abstract}
This expository monograph cuts a short path from the common, elementary background in geometry (linear algebra, vector bundles, and algebraic ideals) to the most advanced theorems about involutive exterior differential systems: (1) The incidence correspondence of the characteristic variety, (2) Guillemin normal form and Quillen's thesis, (3) The Integrability of Characteristics by Guillemin, Quillen, Sternberg, and Gabber, and (4) Yang's Hyperbolicity Criterion. To do so, the geometric theory of PDEs is reinterpreted as the study of smooth sub-bundles of the Grassmann bundle, whereby the rank-1 variety is emphasized. The primary computational tool is an enhanced formulation of Guillemin normal form that is equivalent to involutivity of tableaux.
\end{abstract}

\section{Contents}

0. Introduction and overview $\ldots \ldots \ldots \ldots \ldots \ldots \ldots$

\begin{tabular}{lll}
\hline I Matrices and subspaces & 61
\end{tabular}

1. Tableaux and symbols $\ldots \ldots \ldots \ldots \ldots \ldots$. . . . . . . . . . . . . . . . . . . . . . . . . . . . . . .

1(a). Rank-one ideal . . . . . . . . . . . . . . . . . . . . . . . 61

1(b). Generic bases . . . . . . . . . . . . . . . . . . . . . . . . . . . . . . . . . . . 62

1(c). Endovolutive tableaux . . . . . . . . . . . . . . . . . . 66

1(d). Mutual eigenvectors and rank . . . . . . . . . . . . . . . . . 67

2. Grassmann and universal bundles . . . . . . . . . . . . . . . . . . . . . 68

$2(\mathrm{a}) . \quad$ Tangent and arctangent $\ldots \ldots \ldots \ldots \ldots$

2010 Mathematics Subject Classification: Primary: 58A15; Secondary 35A27, 35A30.

Key words and phrases: characteristic variety, Guillemin normal form, eikonal system. The paper is in final form and no version of it will be published elsewhere. 
2(b). Polar pairs . . . . . . . . . . . . . . . . . . . . . . 70

2(c). The tautological bundle . . . . . . . . . . . . . . . . . 73

\begin{tabular}{ll}
\hline II PDEs on manifolds & $\mathbf{7 4}$
\end{tabular}

3. Bundles upon bundles . . . . . . . . . . . . . . . . . . . . . . . . . . . . . . . . . . . . . . . . . . . . . .

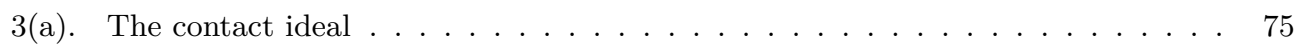

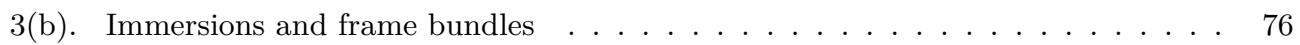

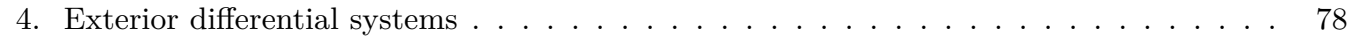

$4(\mathrm{a}) . \quad$ Differential ideals and integral elements . . . . . . . . . . . . . . . . 79

4(b). Prolongation and Spencer cohomology . . . . . . . . . . . . . . . . . . 80

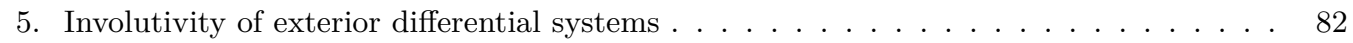

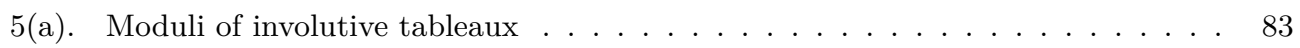

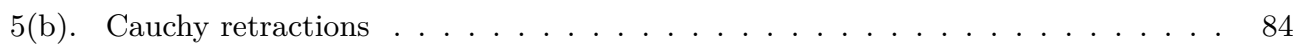

\begin{tabular}{ll}
\hline III Characteristic and rank-one varieties & 84
\end{tabular}

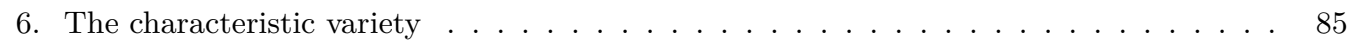

$6(\mathrm{a}) . \quad$ Via polar extension . . . . . . . . . . . . . . . . . . . . . . . . . . . . . . . . . 85

6(b). Via rank-one incidence . . . . . . . . . . . . . . . . . . 86

$6(\mathrm{c}) . \quad$ Example: the wave equation . . . . . . . . . . . . . . . . . . . . . . . . . . . . . .

7. Guillemin normal form and eigenvalues . . . . . . . . . . . . . . . . . . . . . 88

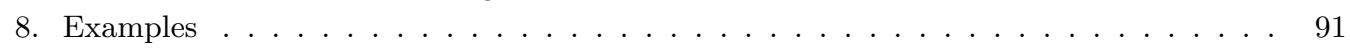

8(a). Zero-dimensional examples . . . . . . . . . . . . . . . . . . . . . . . . . . . . . . . . . 91

8(b). One-dimensional examples . . . . . . . . . . . . . . . . . . . . . . . . . . . . . . . . . . . . . . . . . . . 93

8(c). One-dimensional exercise . . . . . . . . . . . . . . . . . . . . . . . . . . . . . . . . . . . . . . . . .

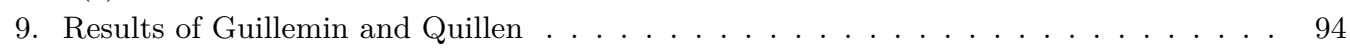

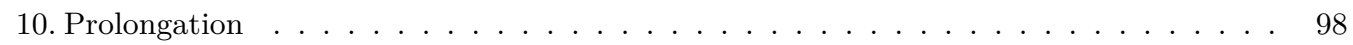

11. Characteristic sheaf . . . . . . . . . . . . . . . . . . . . . . . 98

\begin{tabular}{ll}
\hline IV Eikonal systems & 99
\end{tabular}

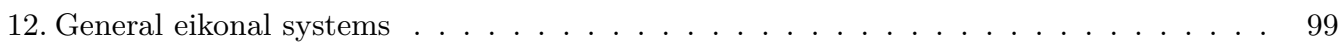

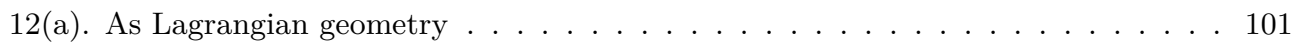

12(b). As Poisson brackets . . . . . . . . . . . . . . . . . . . 102

13. Involutivity of the characteristic variety $\ldots \ldots \ldots \ldots$

14 . Yang's hyperbolicity criterion . . . . . . . . . . . . . . . . . 105

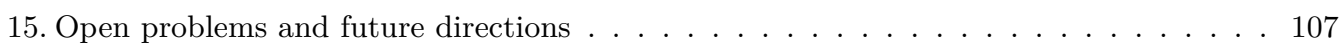

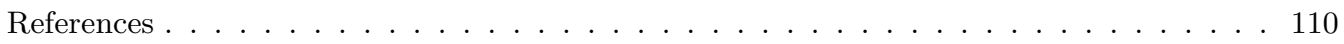

0. Introduction and overview. Given a system of partial differential equations (PDEs) over a manifold, does the system of PDEs have any local solutions to the Cauchy initial-value problem? That is, given initial conditions on a locally-defined hypersurface, can we produce a local solution that satisfies those initial conditions and also satisfies the PDEs? More generally, which initial hypersurfaces admit such solutions? Can we do this iteratively by solving a sequence of initial-value problems from dimension 0 to 1,1 to 2 , and so on to build solutions through any point?

These questions are the heart of exterior differential systems (EDS), a powerful specialist approach to the geometric study of PDEs. EDS typically present as ideals of exterior differential forms over a manifold. 
Some deeper questions are: What is the shape of the family of local solutions obtained in this way? How can we determine whether two systems of PDEs are "the same" up to local coordinate transformations? Does the space of all PDEs (up to local coordinate transformation) have any meaningful shape or structure of its own?

These deeper questions are answered by analyzing the characteristic variety of an EDS. The original motivation for the characteristic variety is to see where the Cauchy initialvalue problem becomes ambiguous. That is, given an initial condition for our PDEs on a local submanifold of dimension $n-1$, when would the $n$-dimensional solutions for that initial condition fail to be unique?

When analyzing the characteristic variety of various EDS, one discovers that the characteristic variety is an exquisitely subtle structure that reveals far more than originally anticipated. The characteristic variety dictates the internal geometry of the solutions of the original PDEs, while also controlling the parameter space of all such solutions. Under reasonable hypotheses, this means that EDS or PDEs can be understood up to coordinate equivalence as "parametrized families of solution manifolds with associated characteristic geometry."

This is beautiful and important, but it has been a difficult topic for researchers to access, because the foundations of EDS have not yet entered the common curriculum of graduate students. Fluency with differential ideals remains a relatively rare skill, practiced in a handful of schools worldwide. Indeed, it is common for researchers first encountering the subject to become trapped in an endless cycle of translating systems from local jet coordinates to differential forms and back again, without gaining any new geometric insights and without using the most powerful theoretical ideas in EDS. In particular, it can take many years for researchers to appreciate the central role that the characteristic variety plays in uncovering geometric insights. However - despite the name - differential forms are not themselves the core idea behind exterior differential systems. Differential forms are merely a concise language. Rather, the core idea is to recognize that these questions are more geometric than analytic, and that ideals (that is, conditions defined by functions) and varieties (that is, shapes cut out by functions) must come into play. To describe families of solutions, we need the geometric language of bundles, schemes, and moduli.

Therefore, the goal of this monograph is to cut the shortest-possible expository path from the common curriculum in geometry (linear algebra, vector bundles, and algebraic ideals) to several key results regarding the characteristic variety. These key results are

(i) the incidence correspondence of the characteristic and rank-1 varieties, and its relationship to eigenspace decomposition,

(ii) Guillemin normal form, its enhancements, and Quillen's thesis,

(iii) the integrability of characteristics (Guillemin, Quillen, Sternberg, Gabber), and

(iv) Yang's hyperbolicity criterion.

The required common curriculum is

(i) graduate-level linear algebra (short-exact sequences, dual spaces, the rank-nullity theorem, tensor products, generalized eigenspaces, as in Artin's Algebra [Art91]), 
(ii) the fundamentals of smooth manifolds (tangent spaces, Sard's theorem, bundles, as in Milnor's Topology from the Differential Viewpoint [Mil97]), and

(iii) basic algebraic geometry (projective space, ideal, variety, scheme, as in Harris' Algebraic Geometry, a first course [Har92]).

To accomplish this, the subject of exterior differential systems is reinterpreted as the study of smooth sub-bundles of the Grassmann bundle over a smooth manifold. In doing so, the role of exterior differential forms becomes obscured, in favor of tableaux (vector spaces of homomorphisms) and symbols (varieties of endomorphisms). Specifically, Guillemin normal form for tableaux and symbols plays the central computational role, not differential forms. This is because most humans - and their computer algebra systems - are more comfortable with matrices than with exterior algebra. Exterior differential ideals are not introduced until absolutely needed. This is because many of the essential lemmas depend only on the geometry of the Grassmann bundle, which is the variety of the trivial exterior differential system. This reformulation allows elementary versions of those key results (in fact, almost all the lemmas are restatements of the rank-nullity theorem), and it becomes possible to outline how these results could be used to push the frontiers of the subject.

While the audience is assumed to have a general interest and cultural awareness of PDEs or EDS in some form, all the required definitions are provided when needed. Even so, it is wise always to have Bryant, Chern, Gardner, Goldschmidt, and Griffiths's Exterior Differential Systems [BCG ${ }^{+}$90] and Ivey and Landsberg's Cartan for Beginners IL03. nearby. They are cited for comparison frequently. Another excellent reference is McKay's Introduction to Exterior Differential Systems [McK18], which appears in the same volume as this monograph. A note for EDS experts: the results in these pages can be found in numerous places in the literature in some form or other, and I have indicated my favorite sources throughout. The only innovation here is in presentation. Most notably, in contrast to the vast majority of expositions, the central topic is the $C^{\infty}$ characteristic variety, not the $C^{\omega}$ Cartan-Kähler theorem. This is because the key open question is "what does the family of all involutive PDEs look like?" not "how do I solve this particular involutive PDE?"

This monograph is organized into four parts, each containing several sections. Part I covers the structure of tableau (subspaces of a space of homomorphisms) and the differential geometry of the Grassmann variety. Because the results are elementary - almost trivially so - they provide a good foundation for building from the common curriculum to the central topic. Part II converts those elementary results to the language of bundles, PDEs and EDS. That language allows an enhanced version of Guillemin normal form that is equivalent to involutivity. Part III achieves the key purpose of this monograph, as a triumvirate is formed by the characteristic scheme, the rank-1 cone, and the mutual eigenvector problem for symbols. Part IV examines the integrability of the characteristic variety in various guises, and offers a general dogma (that the characteristic scheme knows all coordinate-invariant data about a system of PDEs) that suggests possible future developments in the theory of EDS.

This monograph was developed to support a series of lectures at the Institute of 
Mathematics at the Polish Academy of Sciences in September 2016, as part of a Workshop on the Geometry of Lagrangian Grassmannians and Nonlinear PDEs.

\section{Part I. Matrices and subspaces}

1. Tableaux and symbols. Tableaux are very simple objects; every undergraduate encounters the example " $r \times n$ matrices form a vector space using the usual matrix operations," and a tableau is any subspace of that vector space.

Given vector ${ }^{1}$ spaces $W$ and $V$ with $\operatorname{dim} W=r$ and $\operatorname{dim} V=n$, a tableau is a linear subspace of $A \subset \operatorname{Hom}(V, W)$. We use the notation $W \otimes V^{*}$ and $\operatorname{Hom}(V, W)$ interchangeably.

Being a subspace, any tableau $A$ is the kernel of some linear map $\sigma$, called the symbol, whose range is written as $H^{1}(A)$. We have a short exact sequence of spaces:

$$
0 \rightarrow A \rightarrow W \otimes V^{*} \stackrel{\sigma}{\rightarrow} H^{1}(A) \rightarrow 0,
$$

where $H^{1}(A)$ is just notation for $\left(W \otimes V^{*}\right) / A$. Let $\operatorname{dim} A=s$ and $\operatorname{dim} H^{1}(A)=t=n r-s$.

For example, let $W=\mathbb{R}^{3}$ and $V=\mathbb{R}^{3}$, and consider the 5-dimensional tableau $A \subset W \otimes V^{*}$ described in the standard bases by

$$
\left\{\left(\begin{array}{lll}
\alpha_{0} & \alpha_{1} & \alpha_{2} \\
\alpha_{1} & \alpha_{2} & \alpha_{3} \\
\alpha_{2} & \alpha_{3} & \alpha_{4}
\end{array}\right): \alpha_{i} \in \mathbb{R}\right\} .
$$

If $\pi \in W \otimes V^{*}$ is a $3 \times 3$ matrix with entries $\pi_{i}^{a}$, then the symbol $\sigma$ defining $A$ consists of four conditions:

$$
\begin{aligned}
& 0=\pi_{3}^{2}-\pi_{2}^{3}, \\
& 0=\pi_{3}^{1}-\pi_{1}^{3}, \\
& 0=\pi_{2}^{2}-\pi_{1}^{3}, \\
& 0=\pi_{2}^{1}-\pi_{1}^{2} .
\end{aligned}
$$

1(a). Rank-one ideal. The fundamental theorem of linear algebra states that any homomorphism $\pi \in W \otimes V^{*}$ has a well-defined rank. Thus, for any tableau $A \subset W \otimes V^{*}$, we could ask how $\operatorname{rank}(\pi)$ varies across $\pi \in A$. For our purposes, the most interesting ${ }^{2}$ case is $\operatorname{rank}(\pi)=1$.

The space $W \otimes V^{*}$ admits the rank-1 ideal, $\mathscr{R}$, which is irreducible and generated by all $2 \times 2$ minors $\left\{0=\pi_{i}^{a} \pi_{j}^{b}-\pi_{j}^{a} \pi_{i}^{b}\right\}$ in any basis. This is a homogeneous ideal, so we may consider the rank-1 cone in vector space or the rank-1 variety in projective space. (The vertex of the rank-1 cone is the rank-0 matrix.)

For any $A$, consider the ideal $A^{\perp}+\mathscr{R}$, which defines $\mathscr{C} \subset A$ as the variety $\mathscr{C}=$ $A \cap \operatorname{Var}(\mathscr{R})$. The variety $\mathscr{C}$ is the set of matrices in $A$ that are also rank-1; it is a linear section of the rank-1 cone defined by $\mathscr{R}$.

\footnotetext{
${ }^{1}$ When it becomes appropriate to do so, at 4.5 in Section 4 , we switch from vector spaces to complex projective spaces for algebraic convenience.

${ }^{2}$ There is a good reason that the rank-1 case is most interesting: the varieties of higher-rank matrices are determined algebraically by the varieties of lower-rank matrices, so the geometry of $\operatorname{rank}(\pi)$ across $\pi \in A$ comes down to the rank-1 case.
} 
In the example $1.2, \mathscr{C}$ can be parametrized as matrices of the form

$$
\left(\begin{array}{ccc}
\kappa^{4} & \kappa^{3} \tau & \kappa^{2} \tau^{2} \\
\kappa^{3} \tau & \kappa^{2} \tau^{2} & \kappa \tau^{3} \\
\kappa^{2} \tau^{2} & \kappa \tau^{3} & \tau^{4}
\end{array}\right)=\left(\begin{array}{c}
\kappa^{2} \\
\kappa \tau \\
\tau^{2}
\end{array}\right) \otimes\left(\begin{array}{lll}
\kappa^{2} & \kappa \tau & \tau^{2}
\end{array}\right),
$$

which can be interpreted as the rational normal Veronese curve $€^{3}$,

$$
\left[\kappa^{4}: \kappa^{3} \tau: \kappa^{2} \tau^{2}: \kappa \tau^{3}: \tau^{4}\right] \cong \mathbb{P}^{1} \subset \mathbb{P}^{4} \cong \mathbb{P} A .
$$

Moreover, the projection of $\mathscr{C}$ to $\mathbb{P} V^{*}$ is another rational normal curve,

$$
\left[\kappa^{2}: \kappa \tau: \tau^{2}\right] \cong \mathbb{P}^{1} \subset \mathbb{P}^{2} \cong \mathbb{P} V^{*} .
$$

This toy example plays a crucial role in applications for hyperbolic and hydrodynamically integrable PDEs FHK09, Smi09].

1(b). Generic bases. We would like to find a "good" basis in which to express a tableau $A$ and study its properties.

First, an analogy. When studying a single homomorphism $F: \mathbb{C}^{n} \rightarrow \mathbb{C}^{r}$, or $F \in$ $\mathbb{C}^{r} \otimes \mathbb{C}^{n *}$, there are various "good" bases of the domain and co-domain to express $F$. A basis of $\mathbb{C}^{n *}$ is "generic" for $F$ if the first $\operatorname{rank}(F)$ columns are independent. A basis of $\mathbb{F}^{r}$ is "generic" for $F$ if the first $\operatorname{rank}(F)$ rows of $F$ are independent in that basis. Among the generic bases, we can construct particularly "good" bases for writing $F$. When $F$ is written in a "good" basis, we say it is in a "normal form," and the normal form allows us readily to study properties of $F$. For example:

- Use Gaussian elimination 4 to place $F$ in reduced row-echelon form. Then, the rank, kernel, and image of $F$ are immediately apparent. The fundamental theorems in linear algebra depend on this normal form.

- Apply a polar/unitary decomposition to find the singular-value decomposition of $F$. Then, the norm of $F$ and its action with respect to the Hermitian inner products of $\mathbb{C}^{n}$ and $\mathbb{C}^{r}$ are immediately apparent. Important theorems in metric geometry and multivariate statistics depend on this normal form.

- Solve a sequence of eigenvalue problems in the case $n=r$ to find Jordan normal form. Then, the eigenspace structure of $F$, and the commutative algebra of matrices to which it belongs are immediately apparent. The theory of Lie groups and Lie algebras depends on this normal form.

Given a tableau $A \subset W \otimes V^{*}$ with symbol $\sigma$, we are curious whether we can construct bases that are "good" simultaneously for all homomorphisms in the tableau. This situation is considerably more complicated than the situation of a single homomorphism, and it turns out that it is most important to focus on the symbol maps, but we arrive at a satisfying answer in Section 7. By the above analogy, it is convenient to establish a notion of "generic" bases formulated in terms of independence. This is done as follows.

${ }^{3}$ For more on Veronese curves and the more general Segre embeddings and determinantal varieties, see Har92, Sha94.

${ }^{4}$ Algorithmically, this is accomplished using improved Gram-Schmidt or Householder triangularization. See [TB97] for a discussion of stability of row-reduction. 
In any bases of $V^{*}$ and $W$, the tableau $A$ is a space of $r \times n$ matrices only $s$ of whose entries are linearly independent. That is, in a given basis, we can consider the entries $\pi \mapsto \pi_{i}^{a}$ as elements of $A^{*}$, just as we think of the components $v \mapsto v^{i}$ of vectors in $V$ as being linear functions on $v \in \mathbb{R}^{n}$, using the dual basis of $V^{*}$.

Across all bases of $V^{*}$, there is a maximum number of independent entries that can occur in column 1 ; call that number $s_{1}$. (In a measure-zero set of bases of $V^{*}$, the number of actual independent entries in the first column may be less than $s_{1}$.) Once those independent entries are accounted for, there is a maximum number $s_{2}$ of new independent entries that can occur in the second column. (In a measure-zero set of bases of $V^{*}$ that achieve $s_{1}$ in column 1 , the number of actual independent entries in columns 1 and 2 may be less than $s_{1}+s_{2}$.) Once those independent entries are accounted for, there is a maximum number $s_{3}$ of new independent entries that can occur in column 3 . (In a measure-zero set of bases of $V^{*}$ that achieve $s_{1}+s_{2}$ in columns 1 and 2 , the number of actual independent entries in columns 1,2 , and 3 may be less than $s_{1}+s_{2}+s_{3}$.) Continuing in this way, we have $s_{i}$ as the number of new independent entries in the $i$ th column achieved for almost-all bases of $V^{*}$. (In a measure-zero set of bases of $V^{*}$ that achieve $s_{1}+s_{2}+\ldots+s_{i-1}$ in columns 1 through $i-1$, the number of actual independent entries in columns 1 through $i$ may be less than $s_{1}+\ldots+s_{i}$.) Eventually, for such a basis, there is a column $\ell$ where we have reached $s_{1}+s_{2}+\ldots+s_{\ell}=s$, so there is some maximum column $\ell \leq n$ such that $s_{\ell}>0$, where the last independent entry appears. So,

$$
\begin{aligned}
s & =s_{1}+s_{2}+\ldots+s_{\ell}+s_{\ell+1}+\ldots+s_{n} \\
& =s_{1}+s_{2}+\ldots+s_{\ell}+0+\ldots+0 .
\end{aligned}
$$

The index $\ell$ is called the character of $A$, and the number $s_{\ell}$ is called the Cartan integer of $A$. The tuple $\left(s_{1}, \ldots, s_{\ell}\right)$ gives the Cartan characters of $A$. Note that $s_{1} \geq s_{2} \geq \ldots \geq s_{\ell}$, since otherwise the maximality condition would have been violated in an earlier column.

Permanently reserve the index ranges

$$
\begin{aligned}
i, j & \in\{1, \ldots, \ell, \ell+1, \ldots, n\}, \\
\lambda, \mu & \in\{1, \ldots, \ell\}, \\
\varrho, \varsigma & \in\{\ell+1, \ldots, n\}, \text { and } \\
a, b & \in\{1, \ldots, r\} .
\end{aligned}
$$

A basis $5\left(u^{i}\right)=\left(u^{1}, \ldots, u^{n}\right)$ of $V^{*}$ is called generic if its Cartan characters achieve the lexicographical maximum value $\left(s_{1}, s_{2}, \ldots, s_{n}\right)$. As seen in the previous paragraph, almost all bases of $V^{*}$ are generic in this sense. Given a basis $\left(u^{i}\right)$ of $V^{*}$, a basi: ${ }^{6}\left(z_{a}\right)=$ $\left(z_{1}, \ldots, z_{r}\right)$ of $W$ is called generic if the first $s_{i}$ independent entries in column $i$ are independent.

\footnotetext{
${ }^{5}$ We follow the notation of differential geometry. This notation indicates an ordered basis of co-vectors, not a vector. Each $u^{i}$ is an element of $V^{*}$.

${ }^{6}$ We follow the notation of differential geometry. This notation indicates an ordered basis of vectors, not a co-vector. Each $z_{a}$ is an element of $W$.
} 
Choose a generic basis $\left(u^{i}\right)=\left(u^{1}, \ldots, u^{n}\right)$ for $V^{*}$, and let $\left(u_{i}\right)=\left(u_{1}, \ldots, u_{n}\right)$ be its dual basis for $V$. Choose a generic basis $\left(z_{a}\right)=\left(z_{1}, \ldots, z_{r}\right)$ for $W$, and let $\left(z^{a}\right)=$ $\left(z^{1}, \ldots, z^{n}\right)$ be its dual basis for $W^{*}$. An element of the tableau is written as

$$
\pi=\pi_{i}^{a}\left(z_{a} \otimes u^{i}\right) \in W \otimes V^{*},
$$

and the upper-left entries $\pi_{\lambda}^{a}$ for $a \leq s_{\lambda}$ form a basis of $A^{*}$.

Because the bases are generic, the symbol map $\sigma$ can be written as

$$
\left\{0=\pi_{i}^{a}-B_{i, b}^{a, \lambda} \pi_{\lambda}^{b}: 1 \leq i \leq n, s_{i}<a \leq r\right\} .
$$

It is implicit that $B_{i, b}^{a, \lambda}=0$ if $a \leq s_{i}$ or $b \geq s_{\lambda}$ or $i<\lambda$. That is, entries to the lower-right (unshaded) are written as linear combinations of the entries in the upper-left (shaded) using the coefficients $B_{i, b}^{a, \lambda}$, as in Fig. 1 .

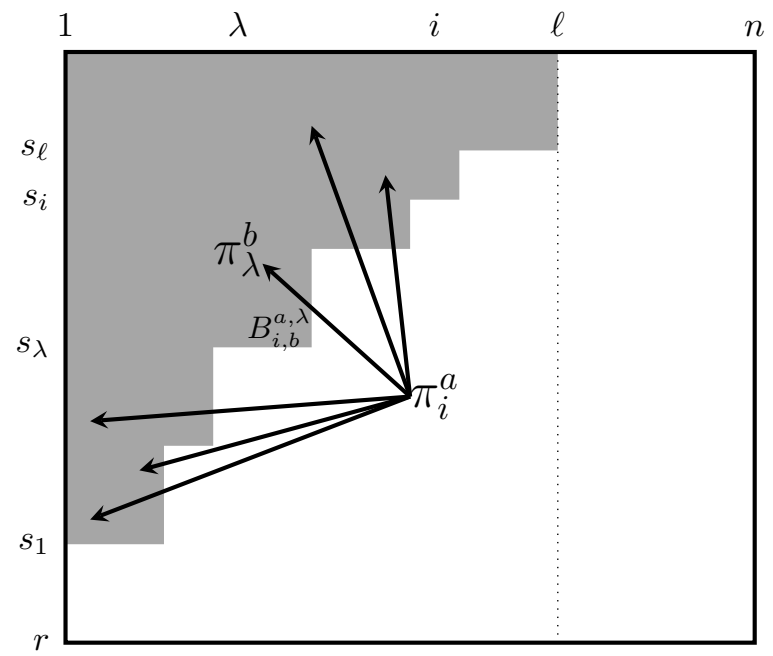

Fig. 1. A tableau in generic bases. Image from Smi15.

Consider the example 1.3 , which is not written in generic bases. If we exchange columns $2 \leftrightarrow 3$ and rows $1 \leftrightarrow 3$, then it becomes generic with $\left(s_{1}, s_{2}, s_{3}\right)=(3,2,0)$, seen here:

$$
\left\{\left(\begin{array}{lll}
\alpha_{2} & \alpha_{4} & \alpha_{3} \\
\alpha_{1} & \alpha_{3} & \alpha_{2} \\
\alpha_{0} & \alpha_{2} & \alpha_{1}
\end{array}\right)\right\}=\left\{\left(\begin{array}{ccc}
\pi_{1}^{1} & \pi_{2}^{1} & \pi_{2}^{2} \\
\pi_{1}^{2} & \pi_{2}^{2} & \pi_{1}^{1} \\
\pi_{1}^{3} & \pi_{1}^{1} & \pi_{1}^{2}
\end{array}\right)\right\} .
$$

Equation 1.10 becomes:

$$
\begin{aligned}
& 0=\pi_{2}^{3}-1 \pi_{1}^{1}-0 \pi_{1}^{2}-0 \pi_{1}^{3}-0 \pi_{2}^{1}-0 \pi_{2}^{2}, \\
& 0=\pi_{3}^{1}-0 \pi_{1}^{1}-0 \pi_{1}^{2}-0 \pi_{1}^{3}-0 \pi_{2}^{1}-1 \pi_{2}^{2}, \\
& 0=\pi_{3}^{2}-1 \pi_{1}^{1}-0 \pi_{1}^{2}-0 \pi_{1}^{3}-0 \pi_{2}^{1}-0 \pi_{2}^{2}, \\
& 0=\pi_{3}^{3}-0 \pi_{1}^{1}-1 \pi_{1}^{2}-0 \pi_{1}^{3}-0 \pi_{2}^{1}-0 \pi_{2}^{2} .
\end{aligned}
$$


One can take the dual perspective, wherein the symbol coefficients $B_{i, b}^{a, \lambda}$ define a map from the upper-left independent entries to the lower-right entries. That is, consider the map

$$
\mathrm{B} \in V^{*} \otimes V \otimes W \otimes W^{*} \cong \operatorname{End}\left(V^{*}\right) \otimes \operatorname{End}(W)
$$

defined by

$$
\sum_{a \leq s_{i}} \delta_{i}^{\lambda} \delta_{b}^{a}\left(z_{a} \otimes z^{b}\right) \otimes\left(u^{i} \otimes u_{\lambda}\right)+\sum_{a>s_{i}} B_{i, b}^{a, \lambda}\left(z_{a} \otimes z^{b}\right) \otimes\left(u^{i} \otimes u_{\lambda}\right) .
$$

Equation (1.14) is the formal inclusion $A \rightarrow W \otimes V^{*}$ in the defining exact sequence (1.1). By fixing $\varphi \in V^{*}$ and $v \in V$, we obtain an endomorphism $\mathrm{B}(\varphi)(v): W \rightarrow W$ defined by the column relations of $\left(\pi_{i}^{a}\right)$, as in Fig. 2 We use the shorthand $\mathrm{B}_{i}^{\lambda}$ for $\mathrm{B}\left(u^{\lambda}\right)\left(u_{i}\right)$, but note that this is not quite the same as $B_{i, b}^{a, \lambda} z_{a} \otimes z^{b}$ because of the identity term in equation 1.14. That is, $\mathrm{B}_{\lambda}^{\lambda}=\sum_{a \leq s_{\lambda}} \delta_{b}^{a}\left(z_{a} \otimes z_{b}\right)$ for all $\lambda \leq \ell$.

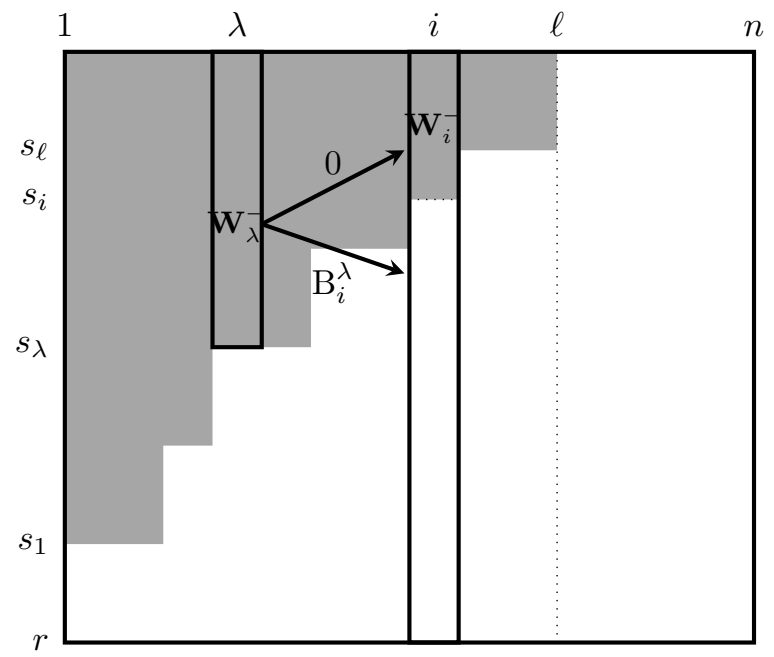

Fig. 2. The map $\mathrm{B}_{i}^{\lambda}$ for a tableau in generic bases. Image from Smi15.

For the example 1.11-1.12, the maps $\mathrm{B}_{i}^{\lambda}: W \rightarrow W$ are:

$$
\begin{aligned}
\mathrm{B}_{1}^{1}=\left(\begin{array}{lll}
1 & 0 & 0 \\
0 & 1 & 0 \\
0 & 0 & 1
\end{array}\right) & \mathrm{B}_{2}^{1}=\left(\begin{array}{lll}
0 & 0 & 0 \\
0 & 0 & 0 \\
1 & 0 & 0
\end{array}\right) & \mathrm{B}_{3}^{1}=\left(\begin{array}{lll}
0 & 0 & 0 \\
1 & 0 & 0 \\
0 & 1 & 0
\end{array}\right) \\
\mathrm{B}_{2}^{2} & =\left(\begin{array}{lll}
1 & 0 & 0 \\
0 & 1 & 0 \\
0 & 0 & 0
\end{array}\right) & \mathrm{B}_{3}^{2}=\left(\begin{array}{lll}
0 & 1 & 0 \\
0 & 0 & 0 \\
0 & 0 & 0
\end{array}\right) .
\end{aligned}
$$

So, if $\varphi=\varphi_{i} u^{i} \in V^{*}$ and $v=v^{j} u_{j} \in V$, the endomorphism $\mathrm{B}(\varphi)(v): W \rightarrow W$ is

$$
\mathrm{B}(\varphi)(v)=\left(\begin{array}{ccc}
\varphi_{1} v^{1}+\varphi_{2} v^{2} & \varphi_{2} v^{3} & 0 \\
\varphi_{1} v^{3} & \varphi_{1} v^{1}+\varphi_{2} v^{2} & 0 \\
\varphi_{1} v^{2} & \varphi_{1} v^{3} & \varphi_{1} v^{1}
\end{array}\right) .
$$


Using our generic basis $\left(u_{i}\right)$ for $V$ and its dual basis $\left(u^{i}\right)$ for $V^{*}$, define decompositions $V=U \oplus Y$ and $V^{*}=Y^{\perp} \oplus U^{\perp}$ using our index convention 1.8 as follows:

$$
\begin{aligned}
V & =\left\langle u_{1}, \ldots, u_{\ell}, u_{\ell+1}, \ldots, u_{n}\right\rangle=\left\langle u_{i}\right\rangle, \\
U & =\left\langle u_{1}, \ldots, u_{\ell} \quad\right\rangle=\left\langle u_{\lambda}\right\rangle, \\
Y & =\left\langle\quad u_{\ell+1}, \ldots, u_{n}\right\rangle=\left\langle u_{\varrho}\right\rangle,
\end{aligned}
$$

and

$$
\begin{aligned}
& V^{*}=\left\langle u^{1}, \ldots, u^{\ell}, u^{\ell+1}, \ldots, u^{n}\right\rangle=\left\langle u^{i}\right\rangle, \\
& U^{*} \cong Y^{\perp}=\left\langle u^{1}, \ldots, u^{\ell} \quad\right\rangle=\left\langle u^{\lambda}\right\rangle, \\
& Y^{*} \cong U^{\perp}=\left\langle\quad u^{\ell+1}, \ldots, u^{n}\right\rangle=\left\langle u^{\varrho}\right\rangle .
\end{aligned}
$$

The isomorphisms $U^{*} \cong Y^{\perp}$ and $Y^{*} \cong U^{\perp}$ depend on the basis; they are non-canonical but sometimes useful.

It is apparent from 1.14 that $\mathrm{B}(\varphi)=\mathrm{B}(\tilde{\varphi})$ if $\varphi-\tilde{\varphi} \in U^{\perp}$; that is if $\varphi_{\varrho}=\tilde{\varphi}_{\varrho}$ for all $\varrho \geq \ell+1$, so we usually consider $\mathrm{B}(\varphi)$ only for $\varphi \in Y^{\perp}$.

Thus, in generic bases, we have a collection $\mathrm{B}_{i}^{\lambda}$ of endomorphisms of $W$. For our purposes of constructing a normal form, a "good" basis is one which makes the endomorphisms $\mathrm{B}_{i}^{\lambda}$ as structurally similar as possible. Section 1(c) imposes additional conditions on the images of these endomorphisms for this purpose.

1(c). Endovolutive tableaux. Suppose $\left(u^{i}\right)$ and $\left(z_{a}\right)$ are generic bases for $A$. For any $i$, define a decomposition $W=\mathbf{W}_{i}^{-} \oplus \mathbf{W}_{i}^{+}$by

$$
\begin{aligned}
W & =\left\langle z_{1}, \ldots, z_{s_{i}}, z_{s_{i}+1}, \ldots, z_{r}\right\rangle=\left\langle z_{a}\right\rangle \\
\mathbf{W}_{i}^{-} & =\left\langle z_{1}, \ldots, z_{s_{i}} \quad\right\rangle \\
\mathbf{W}_{i}^{+} & =\left\langle\quad z_{s_{i}+1}, \ldots, z_{r}\right\rangle .
\end{aligned}
$$

By (1.14, the map $\mathrm{B}_{i}^{\lambda}: W \rightarrow W$ has support $\mathbf{W}_{\lambda}^{-} \subset W$, and its image lies in $\mathbf{W}_{i}^{+} \subset W$.

More generally, for any $\varphi \in V^{*}$, let $\mathbf{W}^{-}(\varphi)=\mathbf{W}_{\underline{\lambda}}^{-}$and $\mathbf{W}^{+}(\varphi)=\mathbf{W}_{\underline{\lambda}}^{+}$, where $\underline{\lambda}$ is the minimum index such that $\varphi_{\underline{\lambda}} \neq 0$. (For general $\varphi$, we have $\operatorname{dim} \mathbf{W}^{-}(\varphi)=s_{1}$.)

A tableau $A$ expressed in bases $\left(u^{i}\right)$ and $\left(z_{a}\right)$ is called endovolutive 7 if $B_{i, b}^{a, \lambda}=0$ for all $a>s_{\lambda}$. That is, endovolutive means that $\mathrm{B}_{i}^{\lambda}$ is an endomorphism of $\mathbf{W}_{\lambda}^{-} \subset W$, as in Fig. 3

Note that the example 1.15 is endovolutive because $s_{2}=2$ and $\mathrm{B}_{2}^{2}$ and $\mathrm{B}_{3}^{2}$ have non-zero entries only in the upper-left $2 \times 2$ part.

${ }^{7}$ The term endovolutive was coined in Smi15, but the phenomenon was described previously in $\mathrm{BCG}^{+} 90$, Chapter IV§5], Yan87, and it is certainly familiar to anyone who has manipulated tableaux of linear Pfaffian systems. 


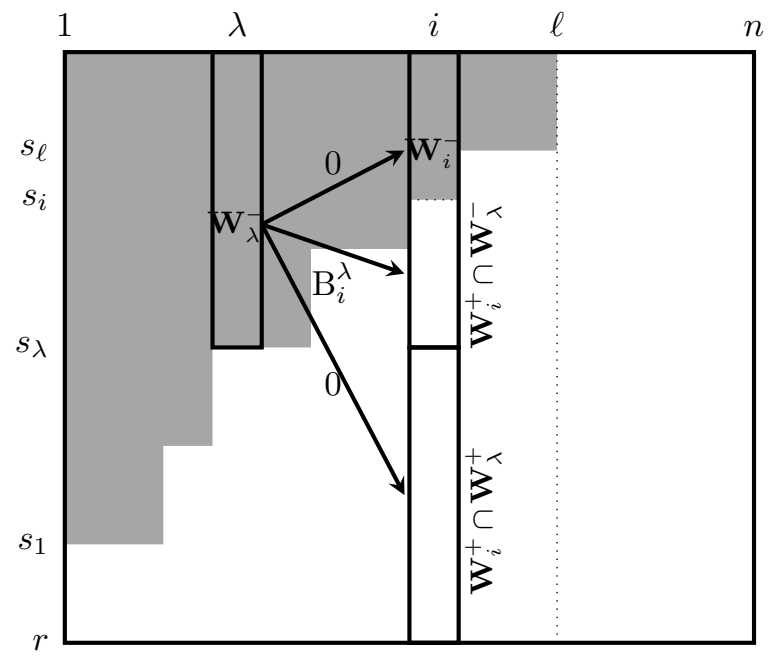

Fig. 3. The map $B_{i}^{\lambda}$ for an endovolutive tableau.

In this way, when considering endovolutive tableaux, it useful to arrange the symbol endomorphisms as an $\ell \times n$ array of $r \times r$ matrices:

$$
\left[\begin{array}{cccccccc}
\mathrm{B}_{1}^{1} & \mathrm{~B}_{2}^{1} & \mathrm{~B}_{3}^{1} & \mathrm{~B}_{4}^{1} & \cdots & \mathrm{B}_{\ell}^{1} & \cdots & \mathrm{B}_{n}^{1} \\
0 & \mathrm{~B}_{2}^{2} & \mathrm{~B}_{3}^{2} & \mathrm{~B}_{4}^{2} & \cdots & \mathrm{B}_{\ell}^{2} & \cdots & \mathrm{B}_{n}^{2} \\
0 & 0 & \mathrm{~B}_{3}^{3} & \mathrm{~B}_{4}^{3} & \cdots & \mathrm{B}_{\ell}^{3} & \cdots & \mathrm{B}_{n}^{3} \\
0 & 0 & 0 & \mathrm{~B}_{4}^{4} & \cdots & \mathrm{B}_{\ell}^{4} & \cdots & \mathrm{B}_{n}^{4} \\
& & & & \ddots & & \mathrm{B}_{i}^{\lambda} & \vdots \\
0 & 0 & 0 & 0 & 0 & \mathrm{~B}_{\ell}^{\ell} & \cdots & \mathrm{B}_{n}^{\ell}
\end{array}\right]
$$

Each "diagonal" entry $\mathrm{B}_{\lambda}^{\lambda}$ is the $r \times r$ matrix for which the non-zero upper-left part is an $s_{\lambda} \times s_{\lambda}$ identity matrix, $I_{s_{\lambda}}$. Endovolutivity means that $\mathrm{B}_{i}^{\lambda}$, which is the $r \times r$ matrix in row $\lambda$ of 1.20 , is zero outside the upper-left $s_{\lambda} \times s_{\lambda}$ part.

If a tableau is endovolutive in certain bases for $W$ and $V^{*}$, then it is also endovolutive under any upper-triangular change-of-basis for $u^{i} \mapsto g_{j}^{i} u^{j}$. Under such a basis change, the columns of $\left(\pi_{i}^{a}\right)$ are linear combinations of the ones to their right, and the sub-matrices in 1.20 change by the corresponding conjugation. Endovolutivity is a property of the flag generated by the basis of $V^{*}$.

1(d). Mutual eigenvectors and rank. For endovolutive bases, each $\mathrm{B}_{i}^{\lambda}$ is an endomorphism of a particular vector space, so it is sensible to consider an eigenvector problem for these maps: For any $\lambda$, let

$$
\mathbf{W}^{1}\left(u^{\lambda}\right)=\left\{w \in \mathbf{W}_{\lambda}^{-} \quad: \quad \mathrm{B}_{\mu}^{\lambda} w=\delta_{\mu}^{\lambda} w, \quad \forall \mu \leq \ell\right\} .
$$

That is, we want to find the vectors that are preserved by $\mathrm{B}_{\lambda}^{\lambda}=I_{s_{\lambda}}$ but are annihilated by all $\mathrm{B}_{\mu}^{\lambda}$ for $\mu \neq \lambda$. More generally, let

$$
\mathbf{W}^{1}(\varphi)=\left\{w \in \mathbf{W}^{-}(\varphi):\left(\sum_{\lambda} \varphi_{\lambda} \mathrm{B}_{\mu}^{\lambda}-\varphi_{\mu} I\right) w=0, \quad \forall \mu \leq \ell\right\} .
$$


Equation 1.22 can be rewritten as a mutual eigenvector problem on the $\ell$ endomorphisms $\mathrm{B}(\varphi)\left(u_{1}\right), \ldots, \mathrm{B}(\varphi)\left(u_{\ell}\right)$ :

$$
\mathbf{W}^{1}(\varphi)=\left\{w \in \mathbf{W}^{-}(\varphi): \mathrm{B}(\varphi)\left(u_{\mu}\right) w=\varphi_{\mu} w, \quad \forall \mu \leq \ell\right\} .
$$

Alternatively, because $B_{\mu}^{\mu}=I_{s_{\mu}}$, equation 1.22 says that $\mathrm{B}(\varphi)(\cdot) w$ is rank-1 when restricted to $Y^{\perp}$, so we can rewrite it as

$$
\mathbf{W}^{1}(\varphi)=\left\{w \in \mathbf{W}^{-}(\varphi): w \otimes \varphi+J_{\varrho}^{a}\left(z_{a} \otimes u^{\varrho}\right) \in A_{e}, \quad \exists J \in W \otimes U^{\perp}\right\} .
$$

This space is the focus of [Gui68], and it plays an important part in our story. Unlike $\mathbf{W}^{-}(\varphi)$, its definition does not depend on the basis; its definition depends only on the splitting $V=U \oplus Y$. Its dimension is an important invariant.

1.25. Lemma. Suppose that the tableau A admits endovolutive bases. For generic $\varphi$, $\operatorname{dim} \mathbf{W}^{1}(\varphi)=s_{\ell}$.

Lemma 1.25 is the result of a quick rank computation using 1.22 - 1.23. See [Smi15].

Our "good" basis and normal form will be built on the requirement that the maps $\mathrm{B}_{i}^{\lambda}$ commute on certain combinations of these spaces (and thus the maps share generalized eigenspaces and Jordan-block normal form there). That is, we are aiming for something like the commutative subalgebras seen in [Ger61] and GS00]. Endovolutivity allows surprisingly direct computation of the desired conditions. For more detail on endovolutivity, see [Smi15] and the references therein. We return to this topic in Section 5, but before that we must introduce the geometry of subspaces.

2. Grassmann and universal bundles. The Grassmann variety is the set $\mathrm{Gr}_{n}(X)$ of $n$-planes in an $(n+r)$-dimensional vector space $X$. It is a smooth projective variety and a smooth manifold of dimension $n r$. An $n$-plane $e \in \operatorname{Gr}_{n}(X)$ is called an element.

2(a). Tangent and arctangent. Depending on one's favorite notation, there are several ways to see that the tangent space of $\operatorname{Gr}_{n}(X)$ at $e$ is $(X / e) \otimes e^{*}$.

First, for any $e \in \operatorname{Gr}_{n}(X)$, choose a basis $\left(u_{i}\right)=\left(u_{1}, \ldots, u_{n}\right)$ for $e$, and choose $\left(z_{a}\right)=\left(z_{1}, \ldots, z_{r}\right)$ so as to complete a basis of the entire vector space $X$. Any $n$-plane $\tilde{e}$ near $e$ admits a basis $\left(\tilde{u}_{i}\right)=\left(\tilde{u}_{1}, \ldots, \tilde{u}_{n}\right)$ that we may assume is related by a matrix in reduced column-echelon form:

$$
\left(\begin{array}{lll}
\tilde{u}_{1} & \ldots & \tilde{u}_{n}
\end{array}\right)=\left(\begin{array}{llllll}
u_{1} & \ldots & u_{n} & z_{1} & \ldots & z_{r}
\end{array}\right)\left(\begin{array}{ccc}
1 & \ldots & 0 \\
& \ddots & \\
0 & \ldots & 1 \\
K_{1}^{1} & \ldots & K_{n}^{1} \\
\vdots & \ddots & \vdots \\
K_{1}^{r} & \ldots & K_{n}^{r}
\end{array}\right) .
$$

More succinctly, using the summation convention:

$$
\tilde{u}_{i}=u_{i}+z_{a} K_{i}^{a}=u_{j} \delta_{i}^{j}+z_{a} K_{i}^{a} .
$$

That is, $\left(\tilde{u}_{i}\right)$ and $\left(u_{i}\right)$ are related by an $(n+r) \times n$ matrix of rank $n$ whose image $\left\langle\tilde{u}_{1}, \ldots, \tilde{u}_{n}\right\rangle=\tilde{e}$ is determined uniquely by the $r \times n \operatorname{submatrix}\left(K_{i}^{a}\right)$. In this sense, 
$T_{e} \operatorname{Gr}_{n}(X)$ is isomorphic to the space of $r \times n$ matrices, which is isomorphic to $(X / e) \otimes e^{*}$. This is easy and computational, but this isomorphism is not canonical for an abstract vector space (without metric) because it depends on a choice of splitting $X=e \oplus(X / e)$ by choosing the complementary basis $\left(z_{a}\right)$.

Alternatively, to see $T_{e} \operatorname{Gr}_{n}(X)=(X / e) \otimes e^{*}$ and avoid splitting, we can use the dua ${ }^{8}$ short-exact sequences

$$
\begin{gathered}
0 \rightarrow e \rightarrow X \rightarrow X / e \rightarrow 0 \\
0 \rightarrow e^{\perp} \rightarrow X^{*} \rightarrow e^{*} \rightarrow 0 .
\end{gathered}
$$

Choose any basis $\left(\theta^{a}\right)=\left(\theta^{1}, \ldots, \theta^{r}\right)$ of the annihilator space $e^{\perp}=(X / e)^{*}$, and let $\left(z_{a}\right)=\left(z_{1}, \ldots, z_{r}\right)$ be the corresponding dual basis of $(X / e)$. Then, we may take the coefficients $K_{i}^{a}$ of

$$
K=z_{a} \otimes K_{i}^{a}(\tilde{e})=z_{a} \otimes \theta^{a}\left(\tilde{u}_{i}\right) \in(X / e) \otimes e^{*}
$$

as $n r$ coordinates on $T_{e} \operatorname{Gr}_{n}(X)$; that is, $K_{i}^{a}$ gives a basis of $T_{e}^{*} \operatorname{Gr}_{n}(X)$.

More abstractly, an explicit choice of bases $\left(u_{i}\right)$ for $e$ and $\left(\theta^{a}\right)$ for $e^{\perp}$ is unnecessary. Instead, we need only the abstract homomorphism $K \in(X / e) \otimes e^{*}$, because the space ${ }^{9}$

$$
\tilde{e}=\left\langle\tilde{u}_{i}\right\rangle=\left\langle u_{i}+K\left(u_{i}\right)\right\rangle=\langle v+K(v): v \in e\rangle
$$

is invariant under $G L(n)$ transformations on $\left(u_{i}\right)$ and $\left(\tilde{u}_{i}\right)$ as well as $G L(r)$ transformations on $\theta$. That is, $\tilde{e}$ is the "graph" of $v \mapsto v+K(v)$ over all $v \in e$.

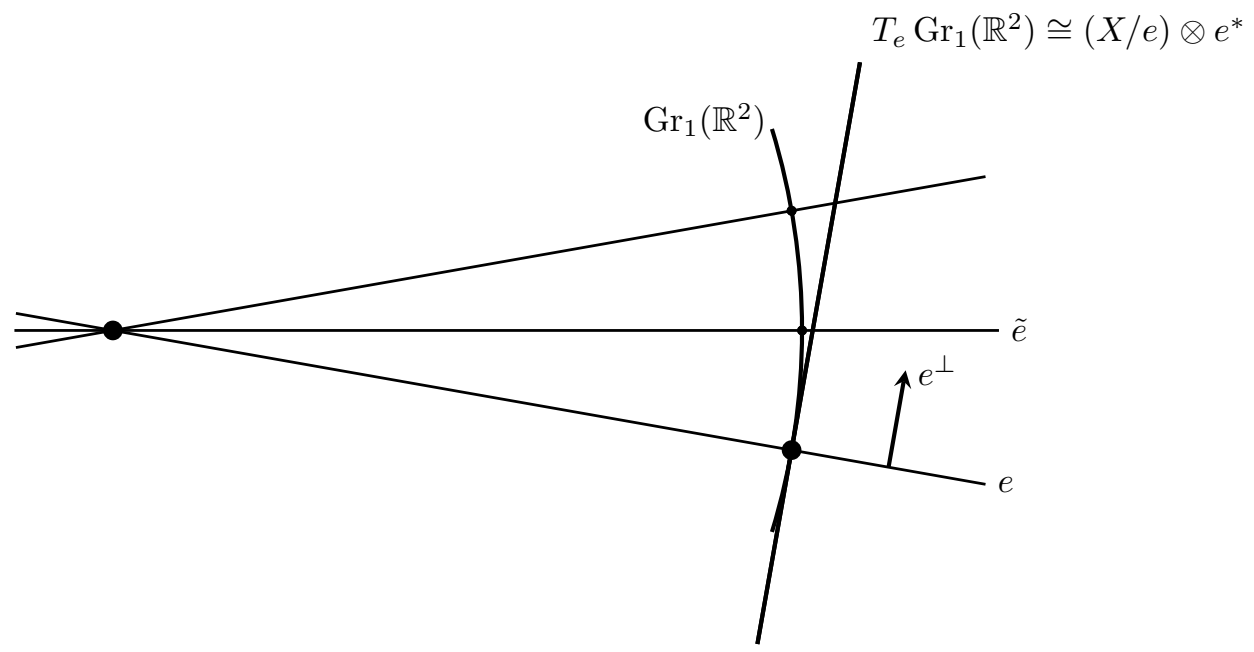

Fig. 4. From $e$, identify a nearby line $\tilde{e}$ in $\mathbb{R}^{2}$ with a relative angle. The map from $T_{e} \mathrm{Gr}_{1}\left(\mathbb{R}^{2}\right) \cong$ $(-\infty, \infty)$ to the neighborhood $(-\pi / 2, \pi / 2)$ of $e$ in $G r_{1}\left(\mathbb{R}^{2}\right)$ is $\arctan _{e}$. Its inverse is $\tan _{e}$.

\footnotetext{
${ }^{8}$ Recall that $(X / e)^{*}$ is canonically isomorphic to $e^{\perp}$ : if $[v]=\{u+e\} \in X / e$, then $\varphi([v])=$ $\varphi(v)+0$ is well-defined for all $\varphi \in e^{\perp}$.

${ }^{9}$ Note that $v+K(v)$ is not well-defined in $X$ for any particular $v \in e$, but the span over all such $v$ is well-defined.
} 
As in Fig. 4, the derivative map $\operatorname{Gr}_{n}(X) \rightarrow(X / e) \otimes e^{*}$ near $e$ is a multidimensional generalization of the tangent function, so the inverse map ${ }^{10}$ is written

$$
\arctan _{e}:(X / e) \otimes e^{*} \rightarrow \operatorname{Gr}_{n}(X) .
$$

The reader is encouraged to read [MS74, §5] and [KN63] and to search for the terms Plücker embedding and Stiefel manifold for more detail on this subject.

2.7. REMARK. Notice that any linear subspace of $(X / e) \otimes e^{*}$ is a tableau in the sense of Section 1. In some sense, it is the only example, as arbitrary $V$ and $W$ could be studied by setting $X=V \oplus W$ and $e=V+0$. Moreover, any smooth submanifold $Z \subset \operatorname{Gr}_{n}(X)$ with tangent space $T_{e} Z \subset T_{e} \operatorname{Gr}(X)$ at $e \in Z$ gives $T_{e} Z$ as a tableau in $(X / e) \otimes e^{*}$. This observation is the heart of the entire subject of exterior differential systems, and it reappears forcefully in Section 4

2(b). Polar pairs. The purpose of this subsection is to establish two results, Lemmas 2.12 and 2.15, that tie the algebraic geometry of intersecting subspaces to the differential geometry of the Grassmannian. These lemmas are used in Part III to demonstrate the correspondence between the characteristic variety (in the Cauchy problem of a system of PDEs) and the rank-1 variety (of the tableau of an EDS) in Lemma 6.4 thus providing the foundation of the geometric theory of PDEs.

Suppose that $e, \tilde{e} \in \operatorname{Gr}_{n}(X)$, and that they intersect along a hyperplane. That is, suppose $e^{\prime}=e \cap \tilde{e}$ and $\operatorname{dim} e^{\prime}=n-1$. We call the pair of $n$-planes $e$ and $\tilde{e}$ a polar pair because they are both polar extensions ${ }^{11}$ of $e^{\prime}$. For any $e \in \operatorname{Gr}_{n}(X)$, let

$$
\operatorname{Pol}_{1}(e)=\left\{\tilde{e} \in \operatorname{Gr}_{n}(X): \operatorname{dim}(\tilde{e} \cap e)=n-1\right\} .
$$

We say $\tilde{e} \in \operatorname{Pol}_{1}(e)$ is a polar pair of e. This relationship is symmetric-hence the unqualified term polar pair - as $\tilde{e} \in \operatorname{Pol}_{1}(e)$ if and only if $e \in \operatorname{Pol}_{1}(\tilde{e})$, but this relationship is not an equivalence relation, as it fails both reflexivity and transitivity.
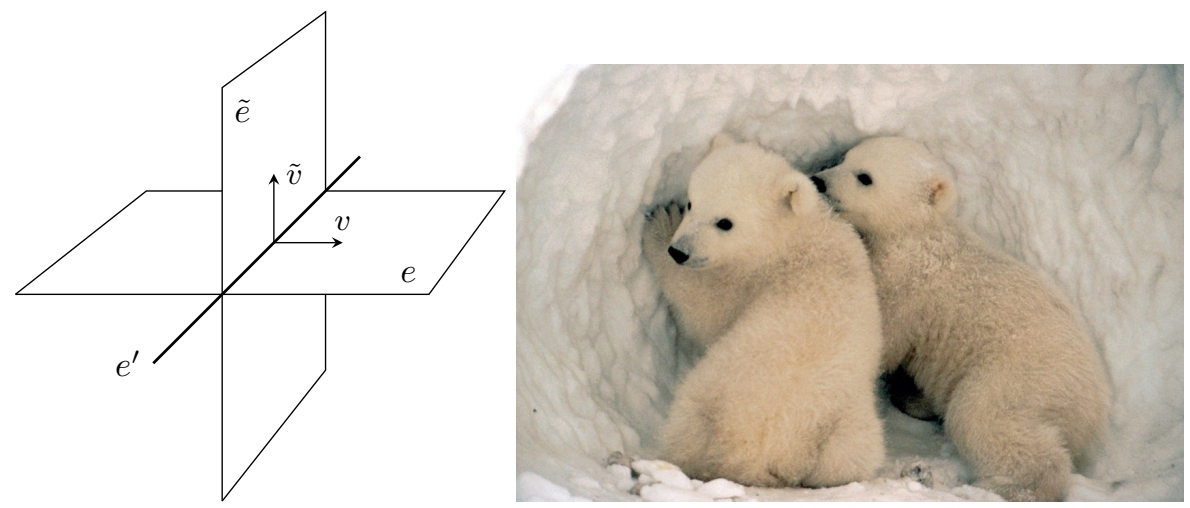

Fig. 5. Polar pairs.

\footnotetext{
${ }^{10}$ The map $\arctan _{e}$ is analogous to exponential map $\exp _{p}: T_{p} M \rightarrow M$ from Riemannian geometry or Lie group representation theory, except that this description of $\arctan _{e}$ does not make explicit use of a metric or group structure.

${ }^{11}$ This is a classical terminology that reappears in Section 6(a)
} 
Within the image of $\arctan _{e}$, Lemma 2.9 ties the notion of polar pairs to lines in the tangent space $T_{e} \operatorname{Gr}_{n}(X)$,

2.9. LemmA. Suppose $e \in \operatorname{Gr}_{n}(X)$ and $\tilde{e}=\arctan _{e}(K)$ for $K \in(X / e) \otimes e^{*}$. Then $\operatorname{rank}(K)=1$ if and only if $\tilde{e} \in \operatorname{Pol}_{1}(e)$.

Proof. Suppose that $\tilde{e} \in \operatorname{Pol}_{1}(e)$. Let $e^{\prime}=e \cap \tilde{e}$, so $\operatorname{dim} e^{\prime}=n-1$. Let $\left(u_{1}, \ldots, u_{n-1}\right)$ be a basis for $e^{\prime}$, and extend that basis to a basis $\left(u_{1}, \ldots, u_{n-1}, v\right)$ for $e$ and to a basis $\left(u_{1}, \ldots, u_{n-1}, \tilde{v}\right)$ for $\tilde{e}$. Writing $(2.2)$ in this case, it is apparent that only the $n$th column of $\left(K_{i}^{a}\right)$ is nonzero. That is, the tangent homomorphism $K \in(X / e) \otimes e^{*}$ is rank-1. (It cannot be the degenerate rank-0 unless $e=\tilde{e}$.)

Conversely, suppose that $K \in(X / e) \otimes e^{*}$ is rank-1. Let $e^{\prime}=\operatorname{ker} K \subset e$, which is a subspace of $e$ of dimension $n-1$. Any line in $e^{\prime}$ is preserved by the map $e \rightarrow X$ defined by the matrix in 2.2); hence, the subspace $e^{\prime}$ is also a subspace of $\tilde{e}$. (It cannot be the degenerate case $e=\tilde{e}$ unless $K$ is rank-0.)

The concept of polar pairs generalizes to co-dimensions $k$ other than 1 . For any $e \in$ $\operatorname{Gr}_{n}(X)$, let

$$
\operatorname{Pol}_{k}(e)=\left\{\tilde{e} \in \operatorname{Gr}_{n}(X): \operatorname{dim}(\tilde{e} \cap e)=n-k\right\} .
$$

Because $\operatorname{dim} X=n+r$ and $\operatorname{dim} e=n$, the set $\mathrm{Pol}_{k}(e)$ is nonempty if and only if $k \leq r$, because $n+k=\operatorname{dim}(e+\tilde{e}) \leq n+r$. The definition is trivial and fairly useless for $k=0$. Again, the $k$-polar-pair relationship $\tilde{e} \in \operatorname{Pol}_{k}(e)$ is symmetric but neither reflexive nor transitive for the interesting case $0<k \leq r$.

One can see immediately that Lemma 2.9 generalizes by replacing 1 with any rank $k$ to give Lemma 2.11

2.11. Lemma. Suppose $e \in \operatorname{Gr}_{n}(X)$ and $\tilde{e}=\arctan _{e}(K)$ for $K \in(X / e) \otimes e^{*}$. Then $\operatorname{rank}(K)=k$ if and only if $\tilde{e} \in \mathrm{Pol}_{k}(e)$.

Next, we can generalize Lemma 2.11 to Lemma 2.12 by dropping the use of arctan. That is, we can consider a $k$-polar pair $(e, \tilde{e})$ where $\tilde{e}$ lies outside the open image of $\arctan _{e}$. From an algebraic perspective, Lemma 2.12 can be seen as a Grassmannian version of the rank-nullity theorem. Phrased in other ways, it is popular true/false homework question in undergraduate linear algebra textbooks.

2.12. Lemma. Fix $e \in \operatorname{Gr}_{n}(X)$ and $\tilde{e} \in \operatorname{Pol}_{k}(e)$. The canonical maps $\tilde{e} \mapsto \tilde{e} / e$ and $\tilde{e} \mapsto(\tilde{e} \cap e)^{\perp} / e^{\perp}$ both have rank-k images, yielding the incidence correspondence Fig. 6 .
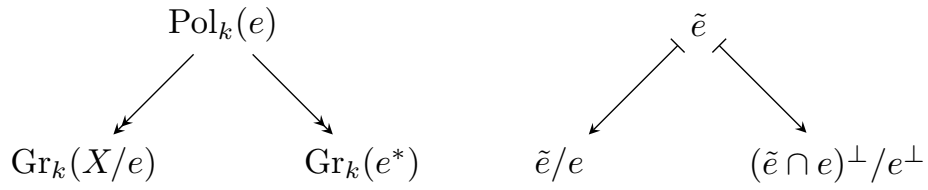

Fig. 6. The incidence correspondence of polar pairs $e$ and $\tilde{e}$.

Proof. Let $e^{\prime}=e \cap \tilde{e}$. Consider the short-exact sequences (2.3), and apply the rank-nullity theorem of those maps on $e^{\prime}$, which has dimension $n-k$. In the first short-exact sequence, $\tilde{e} / e=e^{\prime} / e$ has dimension $k$ as a subspace of $X / e$. In the second short-exact sequence, 
the space $(\tilde{e} \cap e)^{\perp} / e^{\perp}=\left(e^{\prime}\right)^{\perp} / e^{\perp}$ has dimension $k$ as a subspace of $e^{*}=X^{*} / e^{\perp}$. In both cases, and such subspace can be constructed this way.

Now, reconsider the case $k=1$ in light of Lemma 2.12 Then each $\tilde{e} \in \mathrm{Pol}_{1}(e)$ yields a hyperplane $e^{\prime}=\tilde{e} \cap e$. The right image $\left(e^{\prime}\right)^{\perp} / e^{\perp}$ in Fig. 6 is some line $[\xi] \in \mathbb{P} e^{*}$. The left image $\tilde{e} / e$ is some line $[w] \in \mathbb{P}(X / e)$. So, each $\tilde{e} \in \mathrm{Pol}_{1}(e)$ yields a rank-1 projective homomorphism $[w] \otimes[\xi]=[w \otimes \xi] \in \mathbb{P}\left((X / e) \otimes e^{*}\right)$. Any element of $\mathbb{P}\left((X / e) \otimes e^{*}\right)$ could be obtained this way by appropriate choice of $\tilde{e}$.

To see how this generalizes Lemma 2.9 , let us write $[w] \otimes[\xi]$ explicitly. Let

$$
\left(\omega^{1}, \ldots, \omega^{n}, \theta^{1}, \ldots, \theta^{r}\right)
$$

be a basis for $X^{*}$ such that $e=\operatorname{ker}\left\{\theta^{1}, \ldots, \theta^{r}\right\}$ and $e^{\prime}=\operatorname{ker}\left\{\theta^{1}, \ldots, \theta^{r}, \xi\right\}$ for some $\xi=\xi_{i} \omega^{i}$. Then, $\tilde{e}=\operatorname{ker}\left\{\tilde{\theta}^{1}, \ldots, \tilde{\theta}^{r}\right\}$ for some $\tilde{\theta}^{a}=J_{b}^{a} \theta^{b}+K_{i}^{a} \omega^{i}$. Because $e^{\prime} \subset \tilde{e}$, we have

$$
\begin{aligned}
\tilde{\theta}^{a} & \equiv 0 \bmod \left\{\theta^{c}, \xi\right\}, \text { so } \\
J_{b}^{a} \theta^{b}+K_{i}^{a} \omega^{i} & \equiv 0 \bmod \left\{\theta^{c}, \xi\right\}, \text { so } \\
K_{i}^{a} \omega^{i} & \equiv 0 \bmod \left\{\theta^{c}, \xi\right\}, \text { so } \\
K_{i}^{a} \omega^{i} & \equiv 0 \bmod \{\xi\} .
\end{aligned}
$$

Hence, each $K_{i}^{a} \omega^{i}$ is a multiple of $\xi$; call it $w^{a} \xi$. (Note that $w^{a}=0$ for all $a$ if and only if $\tilde{e}=e$, which contradicts our assumption $\operatorname{dim} e^{\prime}=n-1$.) We can use this fact to build a rank-1 homomorphism: Let $\left(z_{a}\right)$ be the basis of $X / e$ dual to $\left(\theta^{a}\right)$. Let $\left(\omega^{i}\right)$ also denote the basis of $e^{*}=X^{*} / e^{\perp}$ induced by $\omega^{i} \in X^{*}$, so that $\xi \in e^{*}$ also denotes the image of $\xi \in X^{*}$. Let $w=w^{a} z_{a}$. Then the induced homomorphism

$$
K=z_{a} \otimes K_{i}^{a} \omega^{i}=z_{a} \otimes w^{a} \xi=w \otimes \xi \in(X / e) \otimes e^{*}
$$

is rank-1. Each of $w$ and $\xi$ is well-defined up to scale, so $K$ is well-defined up to scale, yielding $[K]=\mathbb{P}\left((X / e) \otimes e^{*}\right)$.

It may be that $\tilde{e}$ lies outside the open image of $\arctan _{e}$. How then do we interpret $K$ ? Is there any relationship between $\tilde{e}$ and $\arctan _{e}(K)$ ? From a differential geometric perspective, this is reminiscent of the failure of injectivity at large distances for the exponential map in Riemannian geometry. Lemma 2.15 shows that for any polar pair $\tilde{e}$ of $e$, either $\tilde{e}$ lies in the curve $\arctan _{e}([K])$ or is the limit of the curve.

2.15. Lemma. Suppose $e \in \operatorname{Gr}_{n}(X)$ and $\tilde{e} \in \mathrm{Pol}_{1}(e)$. Then there is a continuous path $\left\{e_{\tau}: 0 \leq 0 \leq 1\right\}$ in $\operatorname{Gr}_{n}(X)$ such that $e_{0}=e, e_{1}=\tilde{e}$, and $e_{\tau} \cap e=\tilde{e} \cap e=e_{\tau} \cap \tilde{e}$ for all $0<\tau<1$. The rank-1 line $[K]$ induced by $e_{\tau}$ via from Lemma 2.12 is constant across $0<\tau \leq 1$. Moreover, $e_{\tau} \in \arctan _{e}([K])$ for $0 \leq \tau<1$.

Proof. Let $e^{\prime}=e \cap \tilde{e}$. For some independent vectors $v, w \in X$, we may write $e=e^{\prime}+\langle v\rangle$ and $\tilde{e}=e^{\prime}+\langle w\rangle$ and define ${ }^{12}$ a curve from $e$ to $\tilde{e}$ in $\operatorname{Gr}_{n}(X)$ by

$$
\left.e_{\tau}=e^{\prime}+\langle(1-\tau) v+\tau w)\right\rangle, \quad 0 \leq \tau \leq 1 .
$$

Note that $e^{\prime}=e \cap e_{\tau}=\tilde{e} \cap e_{\tau}$ for all $0<\tau<1$. It is apparent from $(2.16)$ that $e_{\lambda} / e$ is the line $[\tau w]=[w]$, which is constant versus $\tau$. It is also apparent from $(2.16)$ that

\footnotetext{
${ }^{12}$ If preferred, one can reparametrize from a linear interpolation to a circular interpolation by replacing $\tau$ with $\cos \vartheta$ and $1-\tau$ with $\sin \vartheta$ for some angle $0 \leq \vartheta \leq \pi / 2$.
} 
$\left(e_{\tau} \cap e\right)^{\perp} / e^{\perp}=\left(e^{\prime}\right)^{\perp} / e^{\perp}$ is the line $[\xi]$, which is constant versus $\tau$. Hence, all such $e_{\tau}$ have the same representative rank-1 homomorphism, $[w \otimes \xi]=[K]$ in Lemma 2.12

It may be that $\tilde{e}=e_{1}$ lies outside the open image of $\arctan _{e}$. However, comparison of 2.16 and 2.2 implies that all $e_{\tau}$ lie inside the image of $\arctan _{e}$ for all $\tau<1$. So, the image $\arctan _{e}([w \otimes \xi])$ contains an open set of $\left\{e_{\tau}\right\}$ where $e_{\tau} \cap e=e^{\prime}$.

Consider the example summarized in Fig. 5, where

$$
e=\left\langle\left(\begin{array}{l}
1 \\
0 \\
0
\end{array}\right),\left(\begin{array}{l}
0 \\
1 \\
0
\end{array}\right)\right\rangle \text {, and } \tilde{e}=\left\langle\left(\begin{array}{l}
1 \\
0 \\
0
\end{array}\right),\left(\begin{array}{l}
0 \\
0 \\
1
\end{array}\right)\right\rangle \text {. }
$$

Note that $\tilde{e}$ is outside the open image of $\arctan _{e}$ because (2.2) breaks down as written in this basis. But, $e_{\tau}$ is the family obtained by rotating from $e$ toward $\tilde{e}$ about the axis $e^{\prime}$ through an angle $\arctan \left(\frac{\tau}{1-\tau}\right)$, which varies from 0 to $\frac{\pi}{2}$. For all $0 \leq \tau<1$, we have

$$
e_{\tau}=\left\langle\left(\begin{array}{l}
1 \\
0 \\
0
\end{array}\right),\left(\begin{array}{c}
0 \\
1-\tau \\
\tau
\end{array}\right)\right\rangle=\left\langle\left(\begin{array}{l}
1 \\
0 \\
0
\end{array}\right),\left(\begin{array}{c}
0 \\
1 \\
\frac{\tau}{1-\tau}
\end{array}\right)\right\rangle \text {. }
$$

Thus, the line of rank-1 matrices $[K]$ in $(X / e) \otimes e^{*}$ is written as $\left[\left(\begin{array}{ll}0 & 1\end{array}\right)\right]$ in this basis. This line represented by every $e_{\tau}$ in a curve that converges to $\tilde{e}$ as $\tau \rightarrow 1$. Indeed, up to a choice of basis, this is essentially the only example.

Overall, we have learned that any $k=1$ polar pair in $\operatorname{Gr}_{n}(X)$ is represented by a line of rank-1 matrices in the tangent space, and vice-versa. This is sufficient for our purposes, but those seeking a more detailed understanding of polar pairs are encouraged to investigate Schubert varieties - for example in [Rob14] - and the other outgrowths of Hilbert's 15th problem.

2(c). The tautological bundle. Soon, we will consider algebraic equations defined on $e^{*}$. To facilitate this, for any $e \in \operatorname{Gr}_{n}(X)$, we consider the complexified projective space $\mathbb{X}=\mathbb{P} X \otimes \mathbb{C}$ and its subspace $\mathbb{P} e \otimes \mathbb{C}$. For standard complex projective space, we write $\mathbb{P}^{d}$ for $\mathbb{C P}^{d}=\mathbb{P}\left(\mathbb{C}^{d+1}\right)$. That is, $\mathbb{X} \cong \mathbb{P}^{n+r-1}$, and $\mathbb{P} e \otimes \mathbb{C} \cong \mathbb{P}^{n-1}$.

If we consider all such spaces across all $e$ simultaneously, we obtain the tautological bundl $\AA^{13} \gamma$ over $\operatorname{Gr}_{n}(X)$ with fiber

$$
\gamma_{e}=\mathbb{P} e \otimes \mathbb{C}, \quad \forall e \in \operatorname{Gr}_{n}(X),
$$

and its dual bundle $\gamma^{*}$ over $\operatorname{Gr}_{n}(X)$ with fiber

$$
\gamma_{e}^{*}=\mathbb{P} e^{*} \otimes \mathbb{C}, \quad \forall e \in \operatorname{Gr}_{n}(X),
$$

and its annihilator bundle $\gamma^{\perp}$ over $\operatorname{Gr}_{n}(X)$ with fiber

$$
\gamma_{e}^{\perp}=\mathbb{P} e^{\perp} \otimes \mathbb{C}, \quad \forall e \in \operatorname{Gr}_{n}(X),
$$

and its cokernel bundle $\mathbb{X} / \gamma$ over $\operatorname{Gr}_{n}(X)$ with fiber

$$
(\mathbb{X} / \gamma)_{e}=\mathbb{P}(X / e) \otimes \mathbb{C}, \quad \forall e \in \operatorname{Gr}_{n}(X)
$$

\section{See Fig. 7}

\footnotetext{
${ }^{13}$ These are also called universal bundles or canonical bundles. They are analogous to the sheaves $\mathscr{O}(-1)$ and $\mathscr{O}(1)$, respectively, for varieties in projective space.
} 


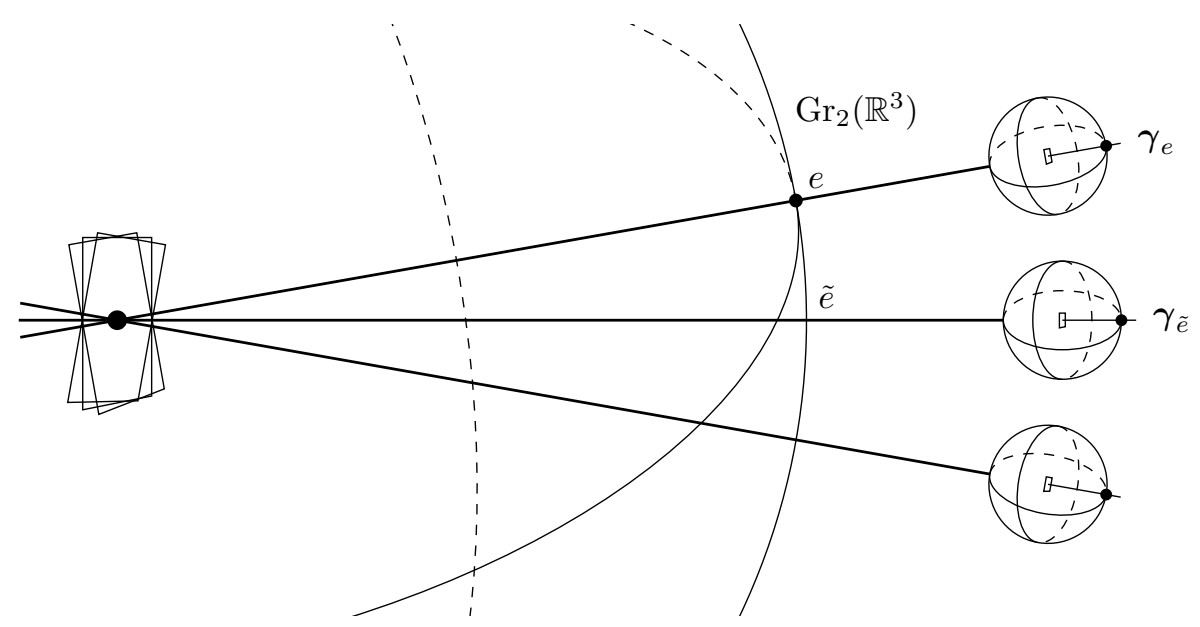

Fig. 7. A cartoon of the tautological bundle, $\gamma$. Here $e$ is a real 2-plane in $\mathbb{R}^{3}$, which can be represented by a line because $G r_{2}\left(\mathbb{R}^{3}\right) \cong \mathbb{P}\left(\mathbb{R}^{3 *}\right)$. Each $\gamma_{e} \cong \mathbb{P}\left(\mathbb{R}^{2}\right) \otimes \mathbb{C}=\mathbb{P}^{1}$ is a Riemann sphere. Thus, $\gamma$ is depicted as a bundle of 2 -spheres over a hemisphere.

There is a dual pair of short exact sequences of projective bundles, analogous to 2.3.

$$
\begin{gathered}
0 \rightarrow \gamma_{e} \rightarrow \mathbb{X} \rightarrow(\mathbb{X} / \gamma)_{e} \rightarrow 0, \\
0 \rightarrow \gamma_{e}^{\perp} \rightarrow \mathbb{X}^{*} \rightarrow \gamma_{e}^{*} \rightarrow 0 .
\end{gathered}
$$

Hence, the complex projectivized tangent bundle $\mathbb{P T} \operatorname{Gr}(X) \otimes \mathbb{C}$ is isomorphic (canonically) to $(\mathbb{X} / \gamma) \otimes \gamma^{*}$. If we choose a splitting of these sequences, then we can use the dual bases to establish a (non-canonical) decomposition $\mathbb{P} X \otimes \mathbb{C} \cong \gamma_{e} \oplus(\mathbb{X} / \gamma)_{e}$ for any $e$.

One can also consider the frame ${ }^{14}$ bundle $\mathcal{F}_{\boldsymbol{\gamma}}$ over $\operatorname{Gr}_{n}(X)$ associated to $\gamma$, whose fiber is all linear isomorphisms

$$
\mathcal{F}_{\boldsymbol{\gamma}, e}=\left\{\left(u^{i}\right): \gamma_{e} \stackrel{\sim}{\rightarrow} \mathbb{P}^{n-1}\right\}=\left\{\text { bases of } \boldsymbol{\gamma}_{e}^{*}\right\} \cong P G L(n),
$$

and the coframe bundle $\mathcal{F}_{\gamma^{*}}$ over $\operatorname{Gr}_{n}(X)$ associated to $\boldsymbol{\gamma}^{*}$, whose fiber is all linear isomorphisms

$$
\mathcal{F}_{\boldsymbol{\gamma}^{*}, e}=\left\{\left(u_{i}\right): \boldsymbol{\gamma}_{e}^{*} \stackrel{\sim}{\rightarrow} \mathbb{P}^{n-1}\right\}=\left\{\text { bases of } \gamma_{e}\right\} \cong P G L(n) .
$$

To write homogeneous complex-algebraic ideals on $\gamma_{e}^{*}$ that vary across $e \in \operatorname{Gr}_{n}(X)$, one can choose any section $\left(u_{i}\right)$ of $\mathcal{F}_{\boldsymbol{\gamma}^{*}}$ to give coordinates, and use the ring

$$
S=C^{\infty}\left(\operatorname{Gr}_{n}(X)\right)\left[u_{1}, \ldots, u_{n}\right] .
$$

\section{Part II. PDEs on manifolds}

In this part, we build bundles whose fibers are the structures seen in Part I] This produces a satisfying language for describing a system of PDEs on a manifold in Section 4

\footnotetext{
${ }^{14}$ Some authors might flip the names of the frame and coframe bundles. I tend to choose this notation because the frame bundle is covariant with diffeomorphisms on the base space, and only contravariant objects get a "co-" prefix. The jargon for duality is always frustrating.
} 
3. Bundles upon bundles. If $M$ is a smooth manifold of dimension $m=n+r$, then we can form the smooth bundle $\operatorname{Gr}_{n}(T M)$ with fiber $\operatorname{Gr}_{n}\left(T_{p} M\right)$. Let $\varpi: \operatorname{Gr}_{n}(T M) \rightarrow M$ denote the bundle projection.

Because 2.3 holds for $X=T_{p} M$ at any $p \in M$, any local section of $\operatorname{Gr}_{n}(T M)$ can be described by choosing its annihilator section of $\operatorname{Gr}_{r}\left(T^{*} M\right)$, and vice-versa. For every $p \in M$, the Grassmann variety $\operatorname{Gr}_{n}\left(T_{p} M\right)$ has a tautological bundle $\gamma(p)$ with fiber $\gamma_{e}(p)=\mathbb{P e} \otimes \mathbb{C}$, a dual bundle, and so on.

The total space $\operatorname{Gr}_{n}(T M)$ is a manifold in its own right, so we may consider $\gamma$ as a bundle over the manifold $\mathrm{Gr}_{n}(T M)$, which is itself a bundle over $M$. In other words, we can reinterpret all of Section $2(\mathrm{c})$ in terms of bundles over $\operatorname{Gr}_{n}(T M)$ by using $\mathbb{X}$ to denote the projective bundle over $\operatorname{Gr}_{n}(T M)$ that has fiber $\mathbb{X}_{e}=\mathbb{P} T_{p} M \otimes \mathbb{C}$ at $e$ with $\varpi(e)=p$. A complete description of some $v \in \gamma$ would be $(p, e, v)$ where $v \in \mathbb{P} e \otimes \mathbb{C}$, and $e \in \operatorname{Gr}_{n}\left(T_{p} M\right)$, and $p \in M$. A complete description of some $\varphi \in \gamma^{*}$ would be $(p, e, \varphi)$ where $\varphi \in \mathbb{P} e^{*} \otimes \mathbb{C}$, and $e \in \operatorname{Gr}_{n}\left(T_{p} M\right)$, and $p \in M$. See Fig. 8 The same bundle-wise constructions hold for $\boldsymbol{\gamma}^{\perp},(\mathbb{X} / \boldsymbol{\gamma}), \mathcal{F}_{\boldsymbol{\gamma}}$, and $\mathcal{F}_{\gamma^{*}}$ from Section $2(\mathrm{c})$

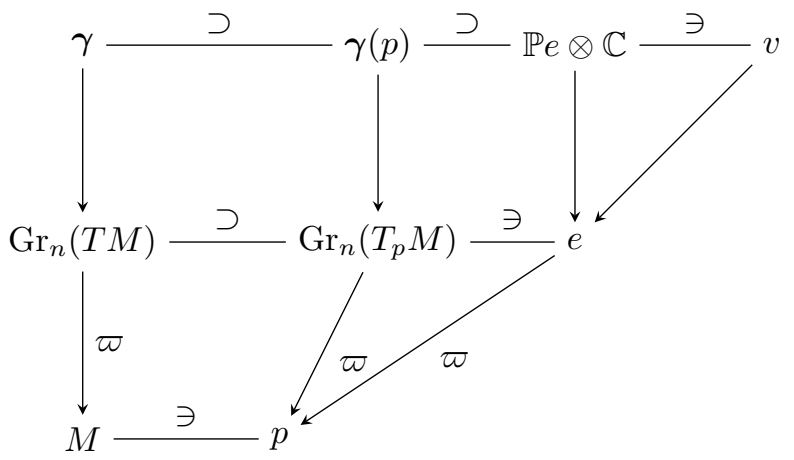

Fig. 8. Tautological bundles over Grassmann bundles over manifolds. Vertical arrows are bundle projections.

Extending 2.26 to write homogeneous complex-algebraic ideals on $\gamma_{e}^{*}$ that vary across $e \in \operatorname{Gr}_{n}(T M)$, one can choose any section $\left(u_{i}\right)$ of $\mathcal{F}_{\boldsymbol{\gamma}^{*}}$ to give coordinates, and use the ring

$$
S=C^{\infty}\left(\operatorname{Gr}_{n}(T M)\right)\left[u_{1}, \ldots, u_{n}\right] .
$$

3(a). The contact ideal. For any $e \in \operatorname{Gr}_{n}(T M)$, consider its annihilator subspace $e^{\perp} \subset T_{p}^{*} M$. There is a corresponding subspace $J_{e} \subset T_{e}^{*} \operatorname{Gr}_{n}(T M)$, defined as

$$
J_{e}=\left\langle\zeta \circ \varpi_{*}: \zeta \in e^{\perp}\right\rangle=e^{\perp} \circ \varpi_{*} .
$$

as in Fig. 9 If $\left(z^{a}\right)$ is a basis of $e^{\perp}$, then we let $\theta^{a}=z^{a} \circ \varpi_{*}$ for each $a$ to define a basis $\left(\theta^{a}\right)$ of $J_{e}$.

In the exterior algebra $\Omega^{\bullet}\left(\mathrm{Gr}_{n}(T M)\right)$, consider the ideal $\mathcal{J}$ that is generated as $\langle J, \mathrm{~d} J\rangle=\left\langle\theta^{a}, \mathrm{~d} \theta^{a}\right\rangle$. This is called the contact ideal, and it is the first example of an EDS as seen in Section 4 . Note that, for any (local) section $\epsilon: M \rightarrow \operatorname{Gr}_{n}(T M)$, the contact ideal satisfies the universal reproducing property

$$
\epsilon^{*}(J)=\epsilon^{*}\left(\epsilon^{\perp} \circ \varpi_{*}\right)=\epsilon^{\perp} \circ \varpi_{*} \circ \epsilon_{*}=\epsilon^{\perp} .
$$


Because this property is universal, the subbundle $J$ is a submodule defined globally across $\mathrm{Gr}_{n}(T M)$ even if topology forces any particular section $\epsilon$ to be defined locally.
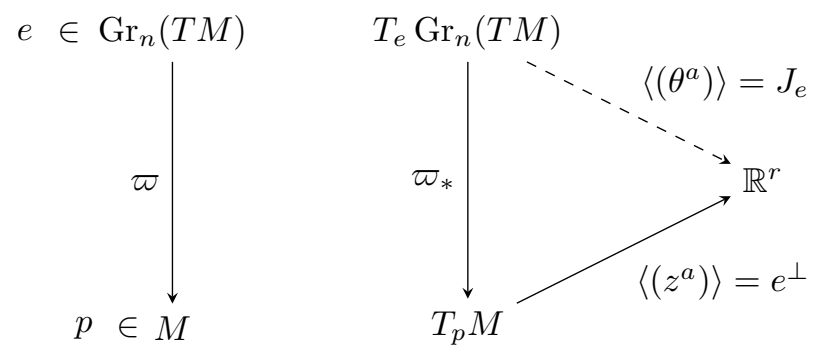

Fig. 9. Contact forms on the Grassmann bundle of $M$.

If one were to choose local coordinates $\left(x^{i}, y^{a}\right)$ for $M$ and local fiber coordinates $\left(P_{i}^{a}\right)$ for $\operatorname{Gr}_{n}(T M)$ near a particular $n$-plane $e=\operatorname{ker}\left\{\mathrm{d} y^{a}\right\}$, then $\mathcal{J}$ is the ideal typically written as

$$
\left\{\begin{array}{l}
0=\theta^{a}=\mathrm{d} y^{a}-P_{i}^{a} \mathrm{~d} x^{i}, \\
0=\mathrm{d} \theta^{a}=-\mathrm{d} P_{i}^{a} \wedge \mathrm{d} x^{i},
\end{array}\right.
$$

where the functions $P_{i}^{a}$ depend on $\tilde{e}$ in an open neighborhood of $e$ in $\operatorname{Gr}_{n}(T M)$.

After reading Section $3(\mathrm{~b})$ compare this coordinate description to your favorite definition of jet space, $\mathbb{J}^{1}\left(\mathbb{R}^{n}, \mathbb{R}^{r}\right)$. Also, compare the local fiber coordinates $P_{i}^{a}$ to the tangent coordinates $K_{i}^{a}$ from Section 2(a) when restricting to the fiber over a single basepoint $p \in M$, they are essentially identical. For some highly amusing applications of the contact system, see Gro86.

3(b). Immersions and frame bundles. Fix an immersion $\iota: N \rightarrow M$ with $\operatorname{dim} N=n$. For any $x \in N$ with $\iota(x)=p$, the push-forward derivative has image $\iota_{*}\left(T_{x} N\right)$, which is an $n$-dimensional subspace of $T_{p} M$; hence, $\iota_{*}\left(T_{x} N\right) \in \operatorname{Gr}_{n}(T M)$. Define the map $\iota^{(1)}: N \rightarrow \mathrm{Gr}_{n}(T M)$ by

$$
\iota^{(1)}(x)=\iota_{*}\left(T_{x} N\right)=e \in \operatorname{Gr}_{n}(T M),
$$

and note that $\iota=\varpi \circ \iota^{(1)}$, so $\iota_{*}=\varpi_{*} \circ \iota_{*}^{(1)}$.

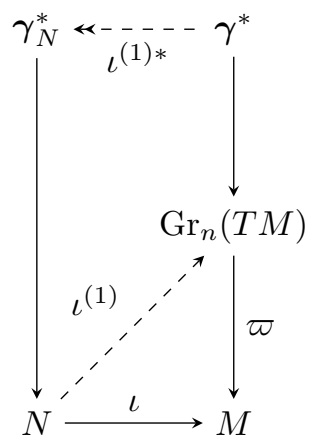

Fig. 10. The dual tautological bundle $\gamma^{*}$ pulls back to form a bundle $\gamma_{N}^{*}$ over an immersed submanifold $N$. 
It is obvious from the definition that $\iota^{(1)}$ is also an immersion. Therefore, we can use it to pull-back the tautological bundle $\gamma^{*}$ as defined in Sections 2(c)] and 3. Let $\gamma_{N}^{*}=\iota^{(1) *} \gamma^{*}$, which has fiber

$$
\gamma_{N, x}^{*}=\gamma_{e}^{*}(p)=\mathbb{P} e^{*} \otimes \mathbb{C}=\mathbb{P}_{\iota_{*}}\left(T_{x} N\right) \otimes \mathbb{C} ;
$$

that is, $\gamma_{N}^{*}$ is identified with $\mathbb{P} T^{*} N \otimes \mathbb{C}$ via $\iota_{*}$. See Fig. 10

The immersion $\iota^{(1)}$ is called the prolongation of the immersion $\iota$.

Now, consider the contact forms $\left(\theta^{a}\right)=\left(z^{a} \circ \varpi_{*}\right)$ forms from Section 3(a), For all $x \in N$ and all $v \in T_{x} N$, we have

$$
\iota^{(1) *}\left(\theta^{a}\right)(v)=\theta^{a}\left(\iota_{*}^{(1)}(v)\right)=z^{a} \circ \varpi_{*} \circ \iota_{*}^{(1)}(v)=z^{a}\left(\iota_{*}(v)\right)=0,
$$

which ultimately gives the following lemma:

3.8. Lemma. If $\iota: N \rightarrow M$ is an immersion for $\operatorname{dim} N=n$, then $\iota^{(1) *}(\mathcal{J})=0$. Conversely, if $\iota^{\prime}: N \rightarrow \operatorname{Gr}_{n}(T M)$ is an immersion for $\operatorname{dim} N=n$ satisfying $\iota^{\prime *}(\mathcal{J})=0$ and such that the image $\iota_{*}^{\prime}\left(T_{x} N\right)$ is transverse to the fiber ker $\varpi_{*}$ for all $x \in N$, then there is some immersion $\iota: N \rightarrow M$ such that $\iota^{(1)}=\iota^{\prime}$.

Moreover, recall that any manifold $N$ of dimension $n$ admits a projective frame bundle $\Pi: \mathcal{F} N \rightarrow N$ with fiber

$$
\mathcal{F}_{x} N=\left\{\left(u^{i}\right): \mathbb{P} T_{x} N \stackrel{\sim}{\rightarrow} \mathbb{P}^{n-1}\right\}=\left\{\text { bases of } \mathbb{P} T_{x}^{*} N \otimes \mathbb{C}\right\} \cong P G L(n),
$$

The total space $\mathcal{F} N$ admits a tautologica ${ }^{15} 1$-form $\omega: T \mathcal{F} N \rightarrow \mathbb{P}^{n-1}$ defined by $\omega_{u}^{i}=$ $u^{i} \circ \Pi_{*}$ as in Fig. 11. It is characterized by its universal reproducing property: for any (local) section $\eta: N \rightarrow \mathcal{F} N$ :

$$
\eta^{*}\left(\omega^{i}\right)=\eta^{*}\left(\eta^{i} \circ \Pi_{*}\right)=\eta^{i} \circ \Pi_{*} \circ \eta_{*}=\eta^{i},
$$

or, more succinctly, $\eta^{*}(\omega)=\eta$.
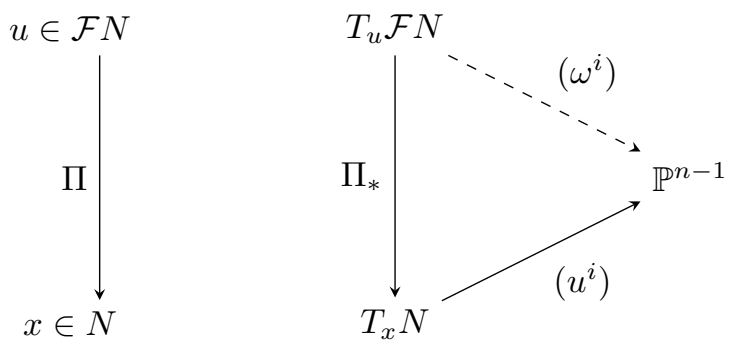

Fig. 11. Tautological form of the frame bundle of a manifold $N$.

Because this property is universal, the 1-form $\omega$ is defined globally across $\mathcal{F} N$ even if topology forces any particular 1-form $\eta$ to be defined locally.

For any local diffeomorphism $f: N \rightarrow \tilde{N}$, there is an induced (covariant) map on the frame bundles $f^{\dagger}: \mathcal{F} N \rightarrow \mathcal{F} \tilde{N}$ by $f^{\dagger}:\left(u^{i}\right) \mapsto\left(u^{i}\right) \circ\left(f_{*}\right)^{-1}$. Using the universal property, it is easy to prove this lemma, which shows that diffeomorphisms are characterized by their preservation of the tautological form on the frame bundle:

\footnotetext{
${ }^{15}$ In various references, this 1 -form is called the canonical, the Hilbert, and the soldering 1 -form.
} 
3.11. Lemma. If $f: N \rightarrow \tilde{N}$ is a diffeomorphism, then $\left(f^{\dagger}\right)^{*}(\tilde{\omega})=\omega$. Conversely, if $F: \mathcal{F} N \rightarrow \mathcal{F} \tilde{N}$ is $P G L(n)$-equivariant diffeomorphism such that $F^{*}(\tilde{\omega})=\omega$, then there exists a unique diffeomorphism $f: N \rightarrow \tilde{N}$ such that $f^{\dagger}=F$.

Combining the universal properties of the $\mathcal{J}$ and $\omega$, we obtain the following theorem telling us what information we can transfer from $\operatorname{Gr}_{n}(T M)$ to an immersed submanifold:

3.12. TheOREM. If $\iota: N \rightarrow M$ is a smooth immersion, then

- $\iota^{(1) *}(\mathcal{J})=0$, and

- $\mathcal{F} N=\iota^{(1) *}\left(\mathcal{F}_{\gamma}\right)$.

Conversely, if $\iota^{\prime}: N \rightarrow \mathrm{Gr}_{n}(T M)$ is a smooth immersion such that

- $\iota^{\prime *}(\mathcal{J})=0$, and

- $\mathcal{F} N=\iota^{\prime *}\left(\mathcal{F}_{\gamma}\right)$,

then there exists a smooth immersion $\iota: N \rightarrow M$ such that $\iota^{(1)}=\iota^{\prime}$.

That is, an immersed submanifold satisfies the contact ideal, which is generated differentially by some annihilator 1-forms $\left(\theta^{a}\right)$ spanning $\gamma^{\perp}$, and its frame bundle is equipped with tautological 1-forms $\left(\omega^{i}\right)$ spanning $\gamma^{*}$.

3.13. REMARK. Note the similarity between the universal property of the contact ideal on the Grassmann bundle and the universal property of the tautological 1-form on the frame bundle. Exploitation of this interaction as in Theorem 3.12 has a long and interesting history.

For example, consider the study of a Lie pseudogroup acting on a manifold $M$. One option is to differentiate the coordinates of $M$ repeatedly using the contact ideal until differential syzygies of the Lie pseudogroup action can be found in prolonged local coordinates, which are then converted to a coordinate-free description using the pseudogroup action. The other option is to work on the frame bundle of $M$ immediately, where any expression on the tautological 1-form is automatically invariant, then prolong as necessary to reveal the syzygies. The latter is used often when the Lie pseudogroup arises as equivalence of intrinsic $G$-structures, and the former is used often when the Lie pseudogroup arises from an extrinsic action on some ambient coordinates. For more on these fascinating and interconnected ideas, I encourage you to read [Cle17, Olv95], Val13], and Gar89] - and the collected works of E. Cartan.

4. Exterior differential systems. Let $M$ be a smooth manifold of finite dimension $m$. An exterior differential system [EDS] on $M$ consists of an ideal $\mathcal{I}$ in the total exterior algebra $\Omega^{\bullet}(M)$ that is differentially closed and finitely generated. Differentially closed means that $\mathrm{d} \mathcal{I} \subset \mathcal{I}$. Finitely generated means that in each degree $d$, the $d$-forms in the ideal, $\mathcal{I}_{d}=\mathcal{I} \cap \Omega^{d}(M)$, form a finitely generated $C^{\infty}(M)$-module. We assume that $\mathcal{I}_{0}=0$; otherwise, one would restrict to a subvariety of $M$ defined by those functions. A solution or integral manifold is an immersed manifold $\iota: N \rightarrow M$ such that $\iota^{*}(\mathcal{I})=0$. Optionally, we sometimes specify an independence condition as an $n$-form $\boldsymbol{\omega} \in \Omega^{n}(M)$ that is not allowed to vanish on solutions. When an EDS represents a system of PDEs in local coordinates $x^{1}, \ldots, x^{n}$, then $\boldsymbol{\omega}=\mathrm{d} x^{1} \wedge \ldots \wedge \mathrm{d} x^{n}$, meaning that we seek solutions $N$ 
on which those coordinates are sensible, and $\iota: N \rightarrow M$ is a function that gives the dependent variables in $M$ (those transverse to $\iota(N) \subset M)$ as functions of the independent variables in $N$.

4.1. REMARK. Exterior differential systems are defined this way because the term "PDE" or "system of PDEs" is difficult to pin down with geometric precision. Colloquially, "system of PDEs" usually means a finite set of (hopefully, smooth) equations on some local jet space. In Section 2, we explored the geometry of the bundle $\operatorname{Gr}_{n}(T M)$; recall that the contact system $\mathcal{J}$ on $\operatorname{Gr}_{n}(T M)$ provides a coordinate-invariant notion of jet space. So, a system of PDEs can be thought of as a collection of equations on jet $\operatorname{Gr}_{n}(T M)$. Hopefully, those equations are smooth and respect the bundle structure coming from the contact system (otherwise, derivatives misbehave). By virtue of the Plücker embedding $\operatorname{Gr}_{n}(T M) \rightarrow \mathbb{P} \wedge^{n}(T M)$, an EDS provides precisely the structure to write an ideal whose variety is a subvariety (in the bundle sense) of $\mathrm{Gr}_{n}(T M)$. By taking smooth subvarieties, we can apply Remark 2.7 and apply our knowledge of tableaux from Part I to study EDS. Even by this definition, an EDS could be rather wild; however, in many practical applications, it happens that $\mathcal{I}$ is generated by a finite collection of smooth differential forms of homogeneous degree, so one obtains a smooth algebraic variety in local fiber coordinates of $\mathrm{Gr}_{n}(T M)$. See [McK18] in this volume for more examples, additional insight, and historical context.

4(a). Differential ideals and integral elements. To be precise, an integral element of $\mathcal{I}$ at $p \in M$ is a linear subspace $e \subset T_{p} M$ such that $\left.\varphi\right|_{e}=0$ for all $\varphi \in \mathcal{I}_{n}$. That is, the $n$-forms in $\mathcal{I}$ provide a collection of functions that cut out a variety, $\operatorname{Var}_{n}(\mathcal{I}) \subset \operatorname{Gr}_{n}(T M)$. These functions vary smoothly in $M$ and are homogeneous in the fiber variables.

There is a maximal dimension $n$ for which $\operatorname{Var}_{n}(\mathcal{I})$ is locally non-empty, which is the case of interest. If an independence condition $\boldsymbol{\omega}$ is specified, we also require $\left.\boldsymbol{\omega}\right|_{e} \neq 0$, which forces $\operatorname{Var}_{n}(\mathcal{I})$ to lie in the open subset of $\operatorname{Gr}_{n}(T M)$ for which that condition holds. (For example, in the case of the contact system, the condition $\boldsymbol{\omega}=\mathrm{d} x^{1} \wedge \ldots \wedge \mathrm{d} x^{n} \neq 0$ holds in the same neighborhood where (3.4 makes sense.)

Because $\mathcal{I}_{n}$ is finitely generated by smooth functions, Sard's theorem guarantees an open, dense subset $\operatorname{Var}_{n}^{o}(\mathcal{I}) \subset \operatorname{Var}_{n}(\mathcal{I})$ defined as the smooth subbundle of $\operatorname{Gr}_{n}(T M)$ that is cut out smoothly by smooth functions.

4.2. Definition (Kähler-ordinary). Integral elements in $\operatorname{Var}_{n}^{o}(\mathcal{I})$ are called Kähler-ordinary.

A single connected component of $\operatorname{Var}_{n}^{o}(\mathcal{I})$ is denoted by $M^{(1)}$. We allow ourselves to redefine $M$ so that $\varpi: M^{(1)} \rightarrow M$ is a smooth bundle.

Let $s$ denote the dimension of each fiber of the projection $M^{(1)} \rightarrow M$, so $t=n r-s$ is the corresponding codimension of $T_{e} M_{p}^{(1)}$ in $T_{e} \operatorname{Gr}_{n}\left(T_{p} M\right)$. That is, the projective bundle $A=\operatorname{ker} \varpi_{*}=T M^{(1)} \subset T \operatorname{Gr}_{n}(T M)$ is a tableau in the sense of Remark 2.7 as each fiber $A_{e}=T_{e} M_{p}^{(1)}$ is a linear subspace of $T_{e} \operatorname{Gr}_{n}\left(T_{p} M\right)$. Because $M^{(1)}$ is a smooth manifold, we have

4.3. Lemma. $K \in A_{e}$ implies $\arctan _{e}(K) \in M^{(1)}$. 
That is, we have a well-defined vector bundle $A=\operatorname{ker} \varpi_{*} \subset T M^{(1)}$ over $M^{(1)}$.

4.4. Definition (Kähler-regular). If $e$ is a Kähler-ordinary integral element and the Cartan characters of each tableau $A$ are constant in an open neighborhood of $e$, then $e$ is called Kähler-regular.

That is, the Kähler-regular integral elements form a dense open subset of the Kähler-

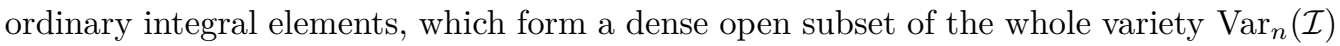
of integral elements.

So that we may apply the results of Section 1 without treating the Cartan characters of $A_{e}$ as functions of $e$, we redefine $M^{(1)}$ to be a single connected component of Kähler-regular integral elements, and we again allow ourselves to redefine $M$ so that $\varpi: M^{(1)} \rightarrow M$ is a smooth bundle.

Such $M^{(1)}$ is called the first prolongation of $(M, \mathcal{I})$, though it is clear from the definition that there could be multiple first prolongations, depending on which components of $\operatorname{Var}_{n}(\mathcal{I})$ are under consideration.

To generalize the notation and results of Part I to $M^{(1)}$, define the restricted tautological bundles

$$
\begin{aligned}
V & =\left.\gamma\right|_{M^{(1)}}=\{\mathbb{P} e \otimes \mathbb{C}\}_{e \in M^{(1)}}, \\
V^{*} & =\left.\gamma^{*}\right|_{M^{(1)}}=\left\{\mathbb{P} e^{*} \otimes \mathbb{C}\right\}_{e \in M^{(1)}}, \\
W & =\left.(\mathbb{X} / \gamma)\right|_{M^{(1)}}=\left\{\mathbb{P}\left(T_{p} M / e\right) \otimes \mathbb{C}\right\}_{e \in M^{(1)}}, \\
V^{\perp} & =\left.\gamma^{\perp}\right|_{M^{(1)}}=\left\{\mathbb{P} e^{\perp} \otimes \mathbb{C}\right\}_{e \in M^{(1)}}
\end{aligned}
$$

WARnING! These are now complex projective bundles, not vector spaces as in Section 1 . Sometimes, it is convenient to think of $A=\operatorname{ker} \varpi^{*} \subset T M^{(1)}$ as being a complex projective bundle, too, in which case we consider it to be a subbundle of the projective bundle $W \otimes V^{*}$. Of course, the notation has been developed to be consistent regardless.

An integral manifold of $\mathcal{I}$ is an immersion $\iota: N \rightarrow M$ such that $\iota^{*}(\varphi)=0$ for all $\varphi \in \mathcal{I}$. (If an independence condition $\boldsymbol{\omega}$ is specified, we require that $\iota^{*}(\boldsymbol{\omega}) \neq 0$, too.) When we are considering a particular Kähler-regular component $M^{(1)} \subset \operatorname{Var}_{n}(\mathcal{I})$ as above, we say $N$ is an ordinary integral manifold provided that $\iota_{*}(T N) \subset M^{(1)}$. All of the observations from Section 3(b) apply, but $\iota^{(1)}(N)$ lies in the submanifold $M^{(1)}$, and $\iota_{*}^{(1)}(T N)$ lies in the subbundle $A$. The overall goal is to construct all ordinary integral manifolds of $(M, \mathcal{I})$ through the careful study of the geometry of a Kähler-regular first prolongation $M^{(1)}$.

4(b). Prolongation and Spencer cohomology. Suppose that $\iota: N \rightarrow M$ is an ordinary integral manifold of $\mathcal{I}$. By Theorem 3.12 the 1 -forms $\theta^{a}$ spanning $J_{e}$ must vanish for each $e \in \iota^{(1)}(N)$. The tautological form $\left(\omega^{i}\right)$ on $\mathcal{F}_{\boldsymbol{\gamma}}$ pulls back to a nondegenerate frame $\left(\eta^{i}\right)$ on $N$, since $\iota^{(1)}$ is an immersion.

Therefore, if $\iota^{(1)}: N \rightarrow M^{(1)}$ actually exists, we have

$$
\begin{aligned}
\iota^{(1) *}\left(\theta^{a}\right) & =0, \\
\iota^{(1) *}\left(\mathrm{~d} \theta^{a}\right) & =0, \\
\iota^{(1) *}\left(\omega^{1} \wedge \ldots \wedge \omega^{n}\right) & =\eta^{1} \wedge \ldots \wedge \eta^{n} \neq 0 .
\end{aligned}
$$


However, working on the frame bundle of $M^{(1)}$, these forms satisfy a more general equation, called Cartan's structure equation:

$$
\mathrm{d} \theta^{a} \equiv \pi_{i}^{a} \wedge \omega^{i}+\frac{1}{2} T_{i, j}^{a} \omega^{i} \wedge \omega^{j} \bmod \left\{\theta^{b}\right\}
$$

The derivative of $\theta^{a}$ must take this form, because $\theta^{a}$ and $\omega^{i}$ are semi-basic with respect to the bundle $\varpi: M^{(1)} \rightarrow M$, whereas $\pi_{i}^{a} \in A$ is vertical, so d $\theta^{a}$ cannot involve a totally vertical 2-form. See discussion of connections and principal bundles in [KN63.

Let us now describe the meaning of each of the terms in (4.7), with respect to the ordinary integral manifold $\iota: N \rightarrow M$. Using the dual coframe $z_{a} \leftrightarrow \theta^{a}$ for $W \leftrightarrow V^{\perp}$, we can see that $\pi=\pi_{i}^{a}\left(z_{a} \otimes \omega^{i}\right)$ lies in $A$. (Hence, it is called the tableau term.) In particular, it must be that

$$
\iota^{(1) *}\left(\pi_{i}^{a}\right)=P_{i, j}^{a} \eta^{j}
$$

for some function $P_{i, j}^{a}$ that must satisfy $P_{i, j}^{a} \eta^{i} \wedge \eta^{j}=0$, so $P_{i, j}^{a}=P_{j, i}^{a}$. That is, the homomorphism $P$ lies in the fiber of $W \otimes\left(V^{*} \otimes V^{*}\right)$ over $e$, as

$$
P \in A \otimes V^{*} \subset\left(W \otimes V^{*}\right) \otimes V^{*}=W \otimes\left(V^{*} \otimes V^{*}\right) .
$$

Moreover, the existence of an immersion $\iota^{(1)}: N \rightarrow M^{(1)}$ requires that the torsion term $w_{a} T_{i, j}^{a} \omega^{i} \wedge \omega^{j}$ can be removed in 4.7); otherwise, it cannot be that $\iota^{(1) *} \mathrm{~d} \theta^{a}=0$ as required. That is, it must be possible to rewrite $\pi_{i}^{a} \hookleftarrow \pi_{i}^{a}+Q_{i, j}^{a} \omega^{j}$ for $Q \in A \otimes V^{*}$ such that any $T_{i, j}^{a}$ term is absorbed. Note that this absorption of torsion is an algebraic property of the tableau $A$. In summary, we have Lemma 4.10

4.10. Lemma. Let $\delta: A \otimes V^{*} \rightarrow W \otimes \wedge^{2} V^{*}$ denote the composition of skewing $\otimes^{2} V^{*} \rightarrow$ $\wedge^{2} V^{*}$ and inclusion $A \rightarrow W \otimes V^{*}$, and write $A^{(1)}=\operatorname{ker} \delta$ and $H^{2}(A)=\operatorname{coker} \delta$ :

$$
0 \rightarrow A^{(1)} \rightarrow A \otimes V^{*} \stackrel{\delta}{\rightarrow} W \otimes \wedge^{2} V^{*} \rightarrow H^{2}(A) \rightarrow 0 .
$$

For any ordinary integral manifold $N$, the homomorphism $P$ of (4.8) and 4.9 lies in $A^{(1)}$, and the pullback of torsion $T$ is zero in $H^{2}(A)$.

Writing $\delta$ in a chosen coframe, it is easy to check that

$$
\operatorname{dim} A^{(1)} \leq s_{1}+2 s_{2}+\ldots+n s_{n} .
$$

The case of equality is considered in Section 5

The exterior differential system $\mathcal{I}^{(1)}$ on $M^{(1)}$ generated as

$$
\mathcal{I}^{(1)}=\left\langle\theta^{a}, \mathrm{~d} \theta^{a}\right\rangle=\varpi^{*}(\mathcal{I})+\mathcal{J}
$$

is called the (first) prolongation of $(M, \mathcal{I})$, and we are back where we started at the beginning of Section 4 We can construct $M^{(2)} \subset \operatorname{Gr}_{n}\left(T M^{(1)}\right)$, and repeat the entire process for $E \in M^{(2)}$ over $e \in M^{(1)}$ that was used for $e \in M^{(1)}$ over $p \in M$. Lemma 4.10 essentially says that $A^{(1)}$ is the tableau bundle $T M^{(2)} \subset T \operatorname{Gr}_{n}\left(T M^{(1)}\right)$. Thus, we can construct $M^{(3)}$ over $M^{(2)}$ and re-apply Lemma 4.10 , and so on. By the definition of $M^{(1)}$ and 4.13, we have

4.14. Corollary. Every ordinary integral manifold $N$ of $\left(M^{(1)}, \mathcal{I}^{(1)}\right)$ is also an ordinary integral manifold of $(M, \mathcal{I})$. However, the converse might fail, as the smooth connected locus of $M^{(1)}$ may be a strict subset of $\operatorname{Var}_{n}(\mathcal{I})$. 
Overall, we achieve exact sequences that summarize the entire situation of the tangent spaces of an immersed ordinary integral manifold $N$ of $\mathcal{I}, \mathcal{I}^{(1)}, \mathcal{I}^{(2)}, \mathcal{I}^{(3)}, \ldots$

$$
\begin{array}{r}
0 \rightarrow A \rightarrow W \otimes \wedge^{1} V^{*} \rightarrow H^{1}(A) \rightarrow 0, \\
0 \rightarrow A^{(1)} \rightarrow A \otimes V^{*} \stackrel{\delta}{\rightarrow} W \otimes \wedge^{2} V^{*} \rightarrow H^{2}(A) \rightarrow 0, \\
0 \rightarrow A^{(2)} \rightarrow A^{(1)} \otimes V^{*} \stackrel{\delta}{\rightarrow} W \otimes \wedge^{3} V^{*} \rightarrow H^{3}(A) \rightarrow 0, \\
\vdots \\
0 \rightarrow A^{(n-1)} \rightarrow A^{(n-2)} \otimes V^{*} \stackrel{\delta}{\rightarrow} W \otimes \wedge^{n} V^{*} \rightarrow H^{n}(A) \rightarrow 0 .
\end{array}
$$

The cokernels $H^{1}(A), H^{2}(A), \ldots, H^{n}(A)$ are the Spencer cohomology of the tableau $A$. Even outside the context of exterior differential systems, they are defined for formal tableaux $A \subset W \otimes V^{*}$ via the exact sequences 4.15 as

$$
H^{k}(A)=\left(A \otimes\left(\otimes^{k-1} V^{*}\right)\right) /\left(W \otimes \wedge^{k} V^{*}\right) .
$$

Spencer cohomology detects functional obstructions to the solution of the initial-value problem on $M^{(k)}$ in the form of torsion; this is explained nicely in [L03, Section 5.6], and the reader is urged to read their presentation.

Spencer cohomology was a major focus of the formal study of partial differential equations and Lie pseudogroups in the mid-20th century; most notably, Spe62, Qui64. SS65, GQS66, Gol67, Gar67, Gui68, GK68, GQS70. As it happens, many of the major results of that era are easy to re-prove under our regularity assumptions on $M^{(1)}$ and using the endovolutive notation from Section 1 , particularly when using the involutivity criteria in Section 5 that were detailed in SSmi15. We demonstrate this in Parts III and IV

\section{Involutivity of exterior differential systems}

5.1. Definition (Cartan's test). A tableau $A$ is called involutive if equality holds in equation 4.12,

$$
s_{1}+2 s_{2}+\ldots+\ell s_{\ell}=\operatorname{dim} A^{(1)} .
$$

5.2. Definition. A tableau $A$ is called formally integrable if $H^{k}(A)=0$ for all $k \geq 2$.

Cartan's test comes from the following consequence of the Cartan-Kähler theorem 16 5.3. THEOREM. Suppose that $(M, \mathcal{I})$ is an analytic exterior differential system, that $M^{(1)}$ is a smooth sub-bundle, and that the tableau bundle $A$ of $r \times n$ homomorphisms has constan ${ }^{17}$ Cartan characters $\left(s_{1}, s_{2}, \ldots, s_{\ell}\right)$ over $M^{(1)}$. If $A$ is involutive and formally integrable, then through any point in $M$, there is an analytic ordinary integral manifold $\iota: N \rightarrow M$. Moreover, such $N$ are parametrized locally by $r$ constants, $s_{1}$ functions of one variable, $s_{2}$ functions of two variables, ..., $s_{\ell}$ functions of $\ell$ variables.

\footnotetext{
${ }^{16} \mathrm{See}\left[\mathrm{BCG}^{+} 90\right.$, Chapter III] or [IL03 for more background on the Cartan-Kähler theorem; it is not our focus here.

${ }^{17}$ That is, $M^{(1)}$ is Kähler-regular.
} 
Somewhat confusingly, the situation in Theorem 5.3 is called involutivity of $(M, \mathcal{I})$; that is, an EDS might fail to be involutive even if its tableau is involutive, because there may be nonzero torsion in $H^{k}(A)$, meaning that $\mathcal{I}$ fails to be formally integrable. This means essentially that the ideal $\mathcal{I}$ is being studied on the wrong manifold.

For a beautiful interpretation of Cartan's test that is relevant to the later sections of this course, read the introduction of [Yan87]. In summary, ordinary integral manifolds are constructed by decomposing the Cauchy problem into a sequence of steps, each of which is determined and has solutions using the Cauchy-Kowalevski theorem.

For fixed spaces $W$ and $V^{*}$, involutivity is a closed algebraic condition on tableaux in $W \otimes V^{*}$. Because the conditions come from Cartan's test, which involves $W \otimes \wedge^{2} V^{*}$, it is not surprising that these conditions are quadratic; however, writing down the precise ideal is a lengthy argument. Doing so was suggested in $\left[\mathrm{BCG}^{+90}\right.$, Chapter IV§5] and accomplished for general tableaux in [Smi15] following the outline in [Yan87.

5.4. THEOREM (Involutivity criteria). Suppose a tableau is given in generic bases as in (1.14). The tableau is involutive if and only if there exists a basis of $W$ such that

(i) $\mathrm{B}_{i}^{\lambda}$ is endovolutive in that basis, and

(ii) $\left(\mathrm{B}_{l}^{\lambda} \mathrm{B}_{k}^{\mu}-\mathrm{B}_{k}^{\lambda} \mathrm{B}_{l}^{\mu}\right)_{b}^{a}=0$ for all $\lambda<l<k$ and $\lambda \leq \mu<k$ and all $a>s_{l}$.

This theorem is our main computational tool in Part III.

5(a). Moduli of involutive tableaux. While it seems like a trivial (if lengthy) computation, consider carefully the meaning of Theorem 5.4. We can fix $r, n$, and Cartan characters $s_{1}, \ldots, s_{n}$ and then write down an explicit ideal in coordinates whose variety is all of the involutive tableaux with those Cartan characters. Hence, we can use computer algebra systems such as Macaulay2, Magma, and Sage to decompose and analyze that ideal using Gröbner basis techniques. With enough computer memory, we can answer the question "What is the moduli of involutive tableaux?" By virtue of Theorem 5.3 this is fairly close to answering the question "What is the moduli of involutive PDEs?".

For example, fix $r=n=3$ and $\left(s_{1}, s_{2}, s_{3}\right)=(3,2,0)$. For some coefficients $x_{0}, \ldots, x_{15}$ in the ring $S$, an endovolutive tableau must be of the form

$$
\left(\pi_{i}^{a}\right)=\left(\begin{array}{ccc}
\alpha_{0} & \alpha_{3} & x_{3} \alpha_{0}+x_{6} \alpha_{1}+x_{9} \alpha_{2}+x_{12} \alpha_{3}+x_{14} \alpha_{4} \\
\alpha_{1} & \alpha_{4} & x_{4} \alpha_{0}+x_{7} \alpha_{1}+x_{10} \alpha_{2}+x_{13} \alpha_{3}+x_{15} \alpha_{4} \\
\alpha_{2} & x_{0} \alpha_{0}+x_{1} \alpha_{1}+x_{2} \alpha_{2} & x_{5} \alpha_{0}+x_{8} \alpha_{1}+x_{11} \alpha_{2}
\end{array}\right),
$$

or in block form like 1.20 ,

$$
\left(\mathrm{B}_{i}^{\lambda}\right)=\left[\begin{array}{ccc}
\left(\begin{array}{lll}
1 & 0 & 0 \\
0 & 1 & 0 \\
0 & 0 & 1
\end{array}\right) & \left(\begin{array}{ccc}
0 & 0 & 0 \\
0 & 0 & 0 \\
x_{0} & x_{1} & x_{2}
\end{array}\right) & \left(\begin{array}{ccc}
x_{3} & x_{6} & x_{9} \\
x_{4} & x_{7} & x_{10} \\
x_{5} & x_{8} & x_{11}
\end{array}\right) \\
\left(\begin{array}{lll}
0 & 0 & 0 \\
0 & 0 & 0 \\
0 & 0 & 0
\end{array}\right) & \left(\begin{array}{lll}
1 & 0 & 0 \\
0 & 1 & 0 \\
0 & 0 & 0
\end{array}\right) & \left(\begin{array}{ccc}
x_{12} & x_{14} & 0 \\
x_{13} & x_{15} & 0 \\
0 & 0 & 0
\end{array}\right)
\end{array}\right] .
$$


Involutivity is an affine quadratic ideal $\mathscr{G}$ on $\mathbb{C}\left(x_{0}, \ldots, x_{15}\right)$ generated by the last rows of $\mathrm{B}_{2}^{1} \mathrm{~B}_{3}^{1}-\mathrm{B}_{3}^{1} \mathrm{~B}_{2}^{1}$ and $\mathrm{B}_{2}^{1} \mathrm{~B}_{3}^{2}-\mathrm{B}_{3}^{1} \mathrm{~B}_{2}^{2}$, so:

$$
\mathscr{G}=\left\{\begin{array}{l}
x_{0} x_{3}+x_{1} x_{4}+x_{2} x_{5}-x_{0} x_{11}, \\
x_{0} x_{6}+x_{1} x_{7}+x_{2} x_{8}-x_{1} x_{11}, \\
x_{0} x_{9}+x_{1} x_{10} \\
x_{0} x_{12}+x_{1} x_{13}-x_{5}, \\
x_{0} x_{14}+x_{1} x_{15}-x_{8} .
\end{array}\right.
$$

The complete primary decomposition of this ideal reveals two components. The maximal component has dimension 12, and it is described by the fairly boring prime ideal $\left\{x_{0}, x_{1}, x_{5}, x_{8}\right\}$. The other component has dimension 11 and its prime ideal is generated by 27 polynomials. See http://goo.gl/jGTnMU for how to compute this in SageMathCell.

Many of your favorite involutive second-order scalar PDEs in three independent variables live somewhere in this variety; see 1.15 and Section 6(c). Up to some notion of equivalence, this is essentially the moduli space of such equations. As seen in Part III] their characteristic varieties are obtained by combining $\mathscr{G}$ with the rank-1 ideal $\mathscr{R}$ on $\mathbb{C}\left[x_{0}, \ldots, x_{15}, a_{0}, \ldots, a_{4}\right]$.

However, there is still some ambiguity to be resolved, as it may be that a given abstract tableau admits several endovolutive bases with apparently distinct coordinate descriptions.

5(b). Cauchy retractions. Before proceeding to Part III it is worthwhile to mention Cauchy retractions, which are much simpler than - and quite distinct from-elements of the characteristic variety. To confuse matters, many references call these "Cauchy characteristics." For any differentially closed ideal $\mathcal{I} \subset \Omega^{\bullet} M$, the Cauchy retractions are the vectors that preserve $\mathcal{I}$; that is, $\mathfrak{g}=\{v \in T M: v\lrcorner \mathcal{I} \subset \mathcal{I}\}$. Because $\mathcal{I}$ is differentially closed, the annihilator bundle $\mathfrak{g}^{\perp} \subset T^{*} M$ is the smallest Frobenius ideal in $\Omega^{\bullet}(M)$ that contains $\mathcal{I}$. Then, for any integral manifold $\iota: N \rightarrow M$, the subspaces $\mathfrak{g} \cap \iota^{(1)}(N)$ form an integrable distribution; that is, $\mathfrak{g}_{N}^{\perp}$ is Frobenius as well [Gar67].

Because $\mathfrak{g}^{\perp}$ is a Frobenius system - a system of ODEs - it is common to redefine $(M, \mathcal{I})$ so that it is free of Cauchy retractions before proceeding to study its integral manifolds. The distinction between $\mathfrak{g}^{\perp}$ and the characteristic variety $\Xi$ is explored further in Smi14.

\section{Part III. Characteristic and rank-one varieties}

Thank you for taking the time to read the enormous amount of background in Parts 1 and III We are ready to define and deconstruct a fascinating mathematical object that lies at the heart of PDE theory.

Here we stand: We have an exterior differential system $\mathcal{I}$ on $M$. Perhaps this EDS arose from a system of PDEs on $M$ and is equipped with an independence condition $\boldsymbol{\omega}$. The EDS yields a smooth Kähler-regular subbundle $M^{(1)} \subset \operatorname{Gr}_{n}(T M)$, where any $e \in M^{(1)}$ is an integral element of the original EDS. As a manifold in its own right, $M^{(1)}$ is equipped 
with tautological bundles $V, V^{*}, W$, and $A$ from 4.5 . Moreover, $A$ is a subbundle of $W \otimes V^{*}$, so it is a tableau bundle. Its symbol $\sigma$ gives a short-exact sequence of bundles,

$$
0 \rightarrow A \rightarrow W \otimes V^{*} \stackrel{\sigma}{\rightarrow} H^{1}(A) \rightarrow 0 .
$$

An integral manifold is an immersion $\iota: N \rightarrow M$ such that $\iota_{*}\left(T_{x} N\right) \in M_{\iota(x)}^{(1)}$ for all $x \in N$. Let $\iota^{(1)}: N \rightarrow M^{(1)}$ denote the map $x \mapsto e=\iota_{*}\left(T_{x} N\right)$.

As you read this part, compare it to [IL03, Section 4.6] and [BCG+90, Chapter V]. The reader will note that we do not assume that $\mathcal{I}$ is a linear Pfaffian system, nor do we build a prolonged $\operatorname{EDS} \mathcal{I}^{(1)}$ using the contact system. Instead we are working with the tautological bundles per Remark 3.13

6. The characteristic variety. The original motivation for the characteristic variety is to see where the initial-value problem becomes ambiguous. That is, given an initial condition for our PDE on a local submanifold of dimension $n-1$, when would the $n$-dimensional solutions for that initial condition fail to be unique? We express this condition in terms of integral elements.

6(a). Via polar extension. For an integral element $e^{\prime} \in \operatorname{Var}_{n-1}(\mathcal{I})$, we consider its space ${ }^{18}$ of integral extensions, called the polar space,

$$
H\left(e^{\prime}\right)=\left\{v: e=e^{\prime}+\langle v\rangle \in \operatorname{Var}_{n}(\mathcal{I})\right\} \subset T M
$$

and the polar equations comprise its annihilator,

$$
\left.H^{\perp}\left(e^{\prime}\right)=\left\{e^{\prime}\right\lrcorner \varphi: \varphi \in \mathcal{I}_{n}\right\} \subset T^{*} M .
$$

The polar rank is $r\left(e^{\prime}\right)=\operatorname{dim} H\left(e^{\prime}\right)-\operatorname{dim} e^{\prime}-1$. If $r\left(e^{\prime}\right)=-1$, then $e^{\prime}$ admits no extensions. If $r\left(e^{\prime}\right)=0$, then $e^{\prime}$ admits a unique extension to some $e \in \operatorname{Var}_{n}(\mathcal{I})$.

The case of interest is $r\left(e^{\prime}\right)>0$, meaning that $e^{\prime}$ admits many extensions, so the initialvalue problem from $e^{\prime}$ to $e=e^{\prime}+\langle v\rangle$ is ambiguous. For any $e \in M^{(1)}$, we can identify a hyperplane $e^{\prime} \in \operatorname{Gr}_{n-1}(e)$ with $\xi \in \mathbb{P} e^{*}$ via $e^{\prime}=\operatorname{ker} \xi$. Because $e \in M^{(1)} \subset \operatorname{Gr}_{n}(T M)$ where $n$ is the maximal dimension of integral elements of $\mathcal{I}$, the function $r$ cannot be positive on an open set of $\mathbb{P} e^{*}$, so the case $r\left(e^{\prime}\right)>0$ is a closed condition. Moreover, the function $r: \mathbb{P} e^{*} \rightarrow \mathbb{N}$ is the rank of a linear system of equations, so it defines a Zariski-closed projective algebraic variety. We choose to study that algebraic variety projectively over $\mathbb{C}$. Hence, the typical definition of the characteristic variety of $e$ is

$$
\Xi_{e}=\left\{\xi \in \mathbb{P} e^{*} \otimes \mathbb{C}: r\left(\xi^{\perp}\right)>0\right\} \subset V_{e}^{*} .
$$

This initial definition is refined in Section 6(b) to produce a scheme. To study properly the ambiguity of the initial-value problem, we want to assign a multiplicity to each $\xi \in \Xi_{e}$ and decompose $\Xi$ into irreducible components based on the structure of the space $H\left(\xi^{\perp}\right)$.

\footnotetext{
${ }^{18}$ The polar space is a vector space thanks to the assumption that $\mathcal{I}_{n}$ is a finitely-generated $C^{\infty}(M)$-module, because that assumption implies that the polar equations over $p \in M$ are a linear subspace of $T_{p}^{*} M$.
} 
6(b). Via rank-one incidence. For both computational and theoretical purposes, it would be convenient to tie the polar space $H\left(e^{\prime}\right)$ to the geometry of the tableau $A_{e}$ of an extension $e$ of $e^{\prime}$. The discussion of polar pairs in Section 2(b) links these two objects, to provide another interpretation of the initial-value problem that is much more convenient than 6.3 .

Fix $e \in M^{(1)}$, and suppose that both $e$ and $\tilde{e}$ are integral extensions of $e^{\prime}=\operatorname{ker} \xi$ for some $\xi \in e^{*}$. By the definition of $H\left(e^{\prime}\right)$, it must be that $\tilde{e}$ lies in $\operatorname{Var}_{n}(\mathcal{I}) \cap \operatorname{Pol}_{1}(e)$, but we do not know whether $\tilde{e}$ lies in the particular maximal smooth component of $\operatorname{Var}_{n}(\mathcal{I})$ that we call $M^{(1)}$. However, the results of Section 2(b) guarantee that $\tilde{e}$ is detected by $A_{e}$ even if $\tilde{e}$ is not in $M^{(1)}$, in the following way.

6.4. Lemma. Fix $e \in M^{(1)}$, and suppose that both $e$ and $\tilde{e}$ are integral extensions of $e^{\prime}=\operatorname{ker} \xi$ for some $\xi \in e^{*}$. Let $w$ be such that $\tilde{e}=e^{\prime}+\langle w\rangle$. Then $w \otimes \xi \in A_{e}$, and there is an open 1-parameter family of integral extensions of $e^{\prime}$ near e in $M^{(1)}$ that also represent $[w \otimes \xi]$.

Proof. Because $\tilde{e} \in \mathrm{Pol}_{1}(e)$, Lemma 2.12 yields a particular line $[K]$ of rank-1 homomorphisms in $\left(T_{p} M / e\right) \otimes e^{*}$ representing $\tilde{e}$. Because $H\left(e^{\prime}\right)$ is a vector space ${ }^{19}$ such that $w \in H\left(e^{\prime}\right)$ and $w \notin e$, the rank-1 projective homomorphism $[K]$ takes the form of $[w \otimes \xi]$ for some $w \in H\left(e^{\prime}\right) / e$.

By Lemma 2.15 there is a continuous one-parameter family of other polar pairs $e_{\tau}$ of $e$, with $e_{\tau} \cap e=e^{\prime}$, converging to $\tilde{e}$, all of which share the rank-1 projective homomorphism $[w \otimes \xi]$.

That is, as a line of rank-1 homomorphisms, $[w \otimes \xi]$ is contained in $\left(H\left(e^{\prime}\right) / e\right) \otimes e^{*}$, as a subspace of $\left(T_{p} M / e\right) \otimes e^{*}$. Applying $\arctan _{e}$, this implies that $e_{\tau} \subset H\left(e^{\prime}\right)$ for all $\tau$. By the definition of $H\left(e^{\prime}\right)$, this means $e_{\tau} \in \operatorname{Var}_{n}(\mathcal{I})$ for all $\tau$. But, the $e_{\tau}$ follow a continuous curve, and $e_{0}=e$ lies in the open subset $M^{(1)}$. Therefore, all $e_{\tau}$ for an open set of sufficiently small $\tau$. Differentiating, we see that the line $[w \otimes \xi]$ is contained in the tangent space of the fiber of $M^{(1)}$ at $e$, namely $A_{e}$.

On the other hand, for fixed $e$ and $\xi$, there are various distinct $\tilde{e}$ corresponding to linearly independent $w$. With Fig. 6 in mind, it is easy to see that

$$
\operatorname{dim} \mathbb{P}\left\{w \in T_{p} M / e: w \otimes \xi \in A_{e}\right\}=r\left(\xi^{\perp}\right) .
$$

Recall the rank-1 ideal $\mathscr{R}$ from Section 1. Here it applies to vector bundles. As a set, the rank-1 subvariety of the tableau is

$$
\mathscr{C}=A \cap \operatorname{Var} \mathscr{R}=A \cap\left\{w \otimes \xi: w \in W, \xi \in V^{*}\right\} .
$$

As a set, the characteristic variety $\Xi$ is the projection of $\mathscr{C}$ to $V^{*}$. More precisely, $\Xi$ is the schem $\mathrm{e}^{20}$ defined by the characteristic ideal $\mathscr{M}$ on $V^{*}$ that is obtained from the rank-1 ideal $\mathscr{R}$ on $A \subset W \otimes V^{*}$ in the following way: For any $\xi \in V^{*}$, define $\sigma_{\xi}: W \rightarrow H^{1}$ by $\sigma_{\xi}(w)=\sigma(w \otimes \xi)$. Note that $\operatorname{dim} \operatorname{ker} \sigma_{\xi}=r\left(\xi^{\perp}\right)$ by (6.5) and (6.6), but this does not

\footnotetext{
${ }^{19}$ Here we see again why it is helpful for an EDS to be finitely generated.

${ }^{20}$ We must study $\Xi$ along with its various components and multiplicities, so it is better to think of it as a scheme than as a simple-minded variety.
} 
account for multiplicity within $\mathscr{C}$ itself. Then the scheme $\mathscr{C}$ is the incidence correspondence $\AA^{21}$ of $\Xi$ for the symbol map $\sigma_{\xi}$. See Fig. 12 .
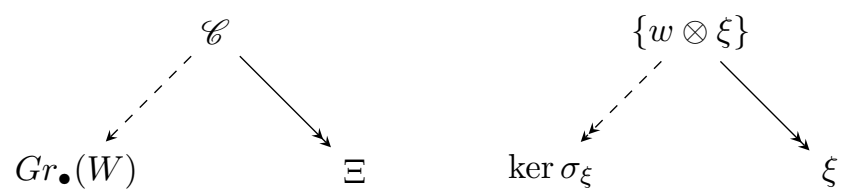

Fig. 12. The rank-1 variety $\mathscr{C}$ is the incidence correspondence for the characteristic variety $\Xi$, but the scheme multiplicities in $\Xi$ should be obtained as in (7.14).

This interpretation is amazing. Suddenly, two completely elementary ideas from Section 1-tableaux of matrices and rank-1 matrices - come together to give a concise description of the most subtle structure in PDE theory.

However, the scheme components and multiplicities are still not obvious from Fig. 12 they must be obtained by examining the degree of the equations defining $\operatorname{ker} \sigma_{\xi}$. The powerful third interpretation in Section 7 provides this detail. But first an example.

6(c). Example: the wave equation. Consider the PDE $f_{11}+f_{22}=f_{33}$. To do this, we consider the manifold $M=\mathbb{R}^{3+1+3+5} \subseteq \mathbb{R}^{13}=\mathbb{J}^{2}\left(\mathbb{R}^{3}, \mathbb{R}\right)$ with coordinates $x^{1}, x^{2}, x^{3}$, $f, p_{1}, p_{2}, p_{3}, p_{11}, p_{12}, p_{13}, p_{22}, p_{23}$. Consider the exterior differential system generated by

$$
\begin{aligned}
& \theta^{0}=\mathrm{d} u-p_{1} \mathrm{~d} x^{1}-p_{2} \mathrm{~d} x^{2}-p_{3} \mathrm{~d} x^{3}, \\
& \theta^{1}=\mathrm{d} p_{1}-p_{11} \mathrm{~d} x^{1}-p_{12} \mathrm{~d} x^{2}-p_{13} \mathrm{~d} x^{3}, \\
& \theta^{2}=\mathrm{d} p_{2}-p_{12} \mathrm{~d} x^{1}-p_{22} \mathrm{~d} x^{2}-p_{23} \mathrm{~d} x^{3}, \\
& \theta^{3}=\mathrm{d} p_{3}-p_{13} \mathrm{~d} x^{1}-p_{23} \mathrm{~d} x^{2}-\left(p_{11}+p_{22}\right) \mathrm{d} x^{3} .
\end{aligned}
$$

Let $\omega^{i}=\mathrm{d} x^{i}$ for $i=1,2,3$, so the derivatives are computed as

$$
\mathrm{d}\left(\begin{array}{c}
\theta^{0} \\
\theta^{1} \\
\theta^{2} \\
\theta^{3}
\end{array}\right) \equiv\left(\begin{array}{ccc}
0 & 0 & 0 \\
\pi_{1}^{1} & \pi_{2}^{1} & \pi_{3}^{1} \\
\pi_{1}^{2} & \pi_{2}^{2} & \pi_{3}^{2} \\
\pi_{1}^{3} & \pi_{2}^{3} & \pi_{3}^{3}
\end{array}\right) \wedge\left(\begin{array}{c}
\omega^{1} \\
\omega^{2} \\
\omega^{3}
\end{array}\right) \bmod \left\{\theta^{0}, \theta^{1}, \theta^{2}, \theta^{3}\right\}
$$

where $\pi_{2}^{1}=\pi_{1}^{2}, \pi_{3}^{1}=\pi_{1}^{3}, \pi_{3}^{2}=\pi_{2}^{3}$, and $\pi_{3}^{3}=\pi_{1}^{1}+\pi_{2}^{2}$.

By changing bases, this tableau is equivalent to an endovolutive one of the form

$$
\left(\pi_{i}^{a}\right)=\left(\begin{array}{ccc}
\alpha_{0} & \alpha_{3} & \alpha_{4} \\
\alpha_{1} & \alpha_{4} & \alpha_{2}+\alpha_{3} \\
\alpha_{2} & \alpha_{0} & \alpha_{1}
\end{array}\right)
$$

\footnotetext{
${ }^{21}$ For more background on the utility of incidence correspondences in algebraic geometry, see the 2013 Columbia Eilenberg lecture series by Joe Harris, Har13. A YouTube link is in the bibliography.
} 
or in block form

$$
\left(\mathrm{B}_{i}^{\lambda}\right)=\left[\begin{array}{lll}
\left(\begin{array}{lll}
1 & 0 & 0 \\
0 & 1 & 0 \\
0 & 0 & 1
\end{array}\right) & \left(\begin{array}{lll}
0 & 0 & 0 \\
0 & 0 & 0 \\
1 & 0 & 0
\end{array}\right) & \left(\begin{array}{lll}
0 & 0 & 0 \\
0 & 0 & 1 \\
0 & 1 & 0
\end{array}\right) \\
\left(\begin{array}{lll}
0 & 0 & 0 \\
0 & 0 & 0 \\
0 & 0 & 0
\end{array}\right) & \left(\begin{array}{lll}
1 & 0 & 0 \\
0 & 1 & 0 \\
0 & 0 & 0
\end{array}\right) & \left(\begin{array}{lll}
0 & 1 & 0 \\
1 & 0 & 0 \\
0 & 0 & 0
\end{array}\right)
\end{array}\right]
$$

Note that the third row of both $\mathrm{B}_{2}^{1} \mathrm{~B}_{3}^{1}-\mathrm{B}_{3}^{1} \mathrm{~B}_{2}^{1}$ and $\mathrm{B}_{2}^{1} \mathrm{~B}_{3}^{2}-\mathrm{B}_{3}^{1} \mathrm{~B}_{2}^{2}$ are zero, so the tableau is involutive by Theorem 5.4

The rank-1 condition is

$$
\begin{aligned}
& 0=\alpha_{0} \alpha_{4}-\alpha_{1} \alpha_{3}, \\
& 0=\alpha_{0} \alpha_{0}-\alpha_{2} \alpha_{3}, \\
& 0=\alpha_{0} \alpha_{1}-\alpha_{2} \alpha_{4}, \\
& 0=\alpha_{1} \alpha_{1}-\alpha_{2} \alpha_{2}-\alpha_{2} \alpha_{3}, \\
& 0=\alpha_{3} \alpha_{1}-\alpha_{0} \alpha_{4}, \\
& 0=\alpha_{3} \alpha_{2}+\alpha_{3} \alpha_{3}-\alpha_{4} \alpha_{4}, \\
& 0=\alpha_{4} \alpha_{1}-\alpha_{0} \alpha_{2}-\alpha_{0} \alpha_{3} .
\end{aligned}
$$

After a simple change of basis, this becomes the example $1.2-(1.4)$, seen throughout the earlier sections.

7. Guillemin normal form and eigenvalues. In this section, we reinterpret $\mathscr{C}$ and $\Xi$ as properties of the endomorphisms $\mathrm{B}_{i}^{\lambda}$. This section is the key to all of the more advanced results that follow. Our main computation tool is the structure of an endovolutive tableau discussed in Section 1(c), where $W$ and $V$ and $A$ are now bundles over $M^{(1)}$.

The incidence correspondence of Fig. 12 is rephrased in Lemma 7.1 .

7.1. LEmmA. If $\xi \in \Xi, v \in V$, and $w \in \operatorname{ker} \sigma_{\xi} \subset W$, then

$$
\mathrm{B}(\xi)(v) w=\xi(v) w .
$$

In particular, $w$ is an eigenvector of $\mathrm{B}(\xi)(v)$ for all $v$.

Proof. Fix generic bases $\left(u^{i}\right)$ and $\left(z_{a}\right)$ and $\left(u_{i}\right)$, so that $\xi=\xi_{i} u^{i}$ and $w=w^{a} z_{a}$ and $v=v^{i} u_{i}$. Set $\pi=w \otimes \xi \in \mathscr{C} \subset A$, so $\pi_{i}^{a}=w^{a} \xi_{i}$ for all $a, i$, and this $\pi$ must satisfy the symbol relations 1.10 . In particular, $w^{a} \xi_{i}=B_{i, b}^{a, \lambda} w^{b} \xi_{\lambda}$ for $a>s_{i}$. Therefore

$$
\begin{aligned}
\mathrm{B}(\xi)(v) w & =\sum_{a \leq s_{i}} \xi_{i} v^{i} w^{a} z_{a}+\sum_{a>s_{i}} B_{i, b}^{a, \lambda} w^{b} \xi_{\lambda} v^{i} z_{a} \\
& =\sum_{a \leq s_{i}} \xi_{i} v^{i} w^{a} z_{a}+\sum_{a>s_{i}} \xi_{i} v^{i} w^{a} z_{a}=\sum_{a, i} \xi_{i} v^{i} w^{a} z_{a}=\xi(v) w .
\end{aligned}
$$

(Here we see the utility of including the first summand in equation (1.14).)

Recalling the decomposition (1.17) and 1.18), Lemma 7.4 provides a sort of converse of Lemma 7.1 
7.4. Lemma. Suppose that $A$ is an endovolutive tableau. Fix $\varphi \in Y^{\perp} \cong U^{*}$ and suppose that $w \in \mathbf{W}^{-}(\varphi)$ is an eigenvector of $\mathrm{B}(\varphi)(v)$ for every $v \in V$. Then there is a $\xi \in \Xi$ over $\varphi \in Y^{\perp}$ such that $w \in \mathbf{W}^{1}(\varphi)$, so $w \otimes \xi \in A$.

Proof. For each $v \in V$, let $\xi(v)$ denote the eigenvalue corresponding to $v$, so that $\xi(v) w=$ $\mathrm{B}(\varphi)(v) w$. Because $\mathrm{B}(\varphi)(v) w$ is linear in $v$, so is $\xi(v)$. Then $\xi=\xi_{i} u^{i} \in V^{*}$. Therefore, $\mathrm{B}(\varphi)(\cdot) w=w \otimes \xi$. In particular, the rank-1 condition implies that

$$
\sum_{\lambda \leq \mu} \varphi_{\lambda} \mathrm{B}_{\mu}^{\lambda} w=\xi_{\mu} w=\sum_{\lambda \leq \mu} \xi_{\lambda} \mathrm{B}_{\mu}^{\lambda} w, \quad \forall \mu \leq \ell .
$$

This is the same expression as in 1.22 , so by comparing recursively over $\mu=1,2, \ldots, \ell$, we see that $\xi_{\lambda}=\varphi_{\lambda}$ for all $\lambda$, so $w \in \mathbf{W}^{1}(\varphi) \subset \mathbf{W}^{-}(\varphi)$.

Lemma 7.4 deserves a warning: There may be multiple $\xi$ over the same $\varphi$, for perhaps there are different eigenvectors $w \in \mathbf{W}^{-}(\varphi)$ admitting different sequences of eigenvalues $\xi_{\varrho}$, for $\varrho>\ell$, associated to the same $\varphi$. Moreover, it is not (yet) clear that a mutual eigenvector $w$ exists for every such $\varphi$.

But overall it is clear that there is some relationship between the eigenvalues of $\mathrm{B}_{i}^{\lambda}$ and the characteristic variety of an endovolutive tableau $A$. This relationship is made precise for involutive tableau using a result from [Gui68].

7.6. THEOREM (Guillemin normal form). Suppose that $A$ is involutive. For every $\varphi \in Y^{\perp}$ and $v \in V$, the restricted homomorphism $\left.\mathrm{B}(\varphi)(v)\right|_{\mathbf{W}^{1}(\varphi)}$ is an endomorphism of $\mathbf{W}^{1}(\varphi)$. Moreover, for all $v, \tilde{v} \in V$,

$$
\left.[\mathrm{B}(\varphi)(v), \mathrm{B}(\varphi)(\tilde{v})]\right|_{\mathbf{W}^{1}(\varphi)}=0 .
$$

Compare Theorem 7.6 to Lemma 4.1 in Gui68 and Proposition 6.3 in Chapter VIII of $\mathrm{BCG}^{+90}$. Theorem 7.6 is known as Guillemin normal form because it implies that the family of homomorphisms $\mathrm{B}(\varphi)(\cdot)$ can be placed in simultaneous Jordan normal form on $\mathbf{W}^{1}(\varphi)$. It is the "normal form" alluded to in Section 1(b) We defer the proof of Theorem 7.6 to Section 9 so we may first see its important consequences.

7.8. COROLlary. If $A$ is involutive, then for each $\varphi \in Y^{\perp}$, there exists some $w$ satisfying the hypotheses of Lemma 7.4. That is, the projection map $\Xi \rightarrow Y^{\perp}$ is onto. In particular, if $A$ is nontrivial and involutive, then $\Xi$ is nonempty.

Proof. Because we are working over $\mathbb{C}$, the commutativity condition (7.7) guarantees that common eigenvectors exist for the commutative algebra $\{\mathrm{B}(\varphi)(v): v \in V\}$.

7.9. Lemma. Suppose that $A$ is an involutive tableau. Then the map of projective varieties induced by $\Xi \rightarrow Y^{\perp}$ is a finite branched cover. In particular, both $\hat{\Xi}$ and $Y^{\perp}$ have affine fiber dimension $\ell$.

Proof. Fix $\varphi \in Y^{\perp}$. The set of $\xi$ over $\varphi$ is nonempty by Corollary 7.8 If it were true that the set of $\xi$ projecting to a particular $\varphi$ were infinite, then the parameter $\xi_{i}$ would take infinitely many values in some expression of the form

$$
\operatorname{det}\left(\sum_{\lambda} \varphi_{\lambda} \mathrm{B}_{i}^{\lambda}-\xi_{i} I\right)=0 .
$$

But, the matrix $\sum_{\lambda} \varphi_{\lambda} \mathrm{B}_{i}^{\lambda} \in \operatorname{End}\left(\mathbf{W}_{1}^{-}\right)$can have at most $s_{1}$ eigenvalues. 
Here we arrive at an easy ${ }^{22}$ proof of the main theorem regarding the structure of $\Xi$. 7.11. Theorem. If $A$ is involutive, then $\operatorname{dim} \Xi=\ell-1$ and $\operatorname{deg} \Xi=s_{\ell}$.

Proof. We work in endovolutive coordinates. From Lemma 7.9. we already know that $\operatorname{dim} \Xi=\ell-1$.

Fix a generic point $\xi \in \Xi$ over $\varphi \in Y^{\perp}$. Let $\mathscr{C}_{\xi}=\left(\operatorname{ker} \sigma_{\xi}\right) \otimes \xi$ denote the fiber over $\xi$ in $\mathscr{C}$. To understand the scheme $\Xi$, we must determine the degree of the condition defining $\mathscr{C}_{\xi}$. Note that $\mathscr{C}_{\xi}$ must be a subvariety of $\mathbf{W}^{1}(\varphi) \otimes \xi$, and $\mathbf{W}^{1}(\varphi)$ is a linear subspace of $W$, so the degree of $\Xi$ is the degree of some condition on $\mathbf{W}^{1}(\varphi)$.

By Lemma 7.1 and 6.6), the condition that $\mathscr{C}_{\xi}$ is nontrivial is precisely the condition that

$$
\operatorname{det}\left(\sum_{\lambda} \xi_{\lambda} \mathrm{B}_{i}^{\lambda}-\xi_{i} I\right)=0, \quad \forall i .
$$

Since we may restrict our attention to $\mathbf{W}^{1}(\varphi) \otimes \xi$, the condition 7.12 for $i \leq \ell$ is automatic by 1.23 . Hence, only these terms contribute to the non-linear part of the ideal:

$$
\operatorname{det}\left(\sum_{\lambda} \xi_{\lambda} \mathrm{B}_{\varrho}^{\lambda}-\xi_{\varrho} I\right)=0, \quad \forall \varrho>\ell
$$

So, without coordinates, the defining equations of $\mathscr{C}_{\xi}$ are

$$
\operatorname{det}(\mathrm{B}(\xi)(v)-\xi(v) I)=0, \quad \forall v \in Y .
$$

For a particular $v$, this is the characteristic polynomial of $\mathrm{B}(\xi)(v)$ as an endomorphism of $\mathbf{W}^{1}(\varphi)$. By involutivity and Theorem 7.6 , all $\mathrm{B}(\xi)(v)$ for $v \in Y$ admit the same Jordanblock form, so they admit the same factorization type for their respective characteristic polynomials. That means it suffices to consider a single $v$. By definition, the characteristic polynomial of $\left.\mathrm{B}(\xi)(v)\right|_{\mathbf{W}^{1}(\varphi)}$ has degree $\operatorname{dim} \mathbf{W}^{1}(\varphi)$ at generic $\varphi$. Therefore, $\operatorname{deg} \Xi=s_{\ell}$ follows from Lemma 1.25 .

Theorems 7.6 and 7.11 provide a powerful interpretation of the form of an involutive tableau seen in Theorem 5.4 and Fig. 3 the first $\ell$ columns represent a projection of $\Xi$, as in Lemma 7.9 and the rank-1 incidence correspondence in Fig. 12 is precisely the eigenvector condition on the appropriate subspaces. It is peculiar and interesting that these results were discovered in the opposite order historically, as explored in Section 9.

The proof of Theorem 7.11 in particular equation (7.14 - gives a precise understanding of $\Xi$ as a scheme. Specifically, the characteristic scheme (in the sense of PDE) is merely a scheme of characteristic equations (in the sense of linear algebra)! The components of $\Xi$ correspond to the various Jordan blocks apparent in $(7.14)$. The multiplicity of

\footnotetext{
${ }^{22}$ It is easy in the sense that we have the explicit polynomials of $\mathscr{M}$ in hand, and they are recognizable as the familiar eigenvector equations. The reader should compare (7.14) to the descriptions provided in $\mathrm{BCG}^{+} 90$ and [L03. Both references defer their decomposition of $\Xi$ to the abstract Grothendieck-Riemann-Roch theorem. Hence, neither reference indicates how to compute the scheme by hand for general tableaux. While details are given in $\mathrm{BCG}^{+} 90$ in the simple case of rectangular tableaux, a complete description is achieved here because of the normal form provided by Theorem 5.4 .
} 
each component is the dimension of that generalized eigenspace. The sheets of the finite branched cover $\Xi \rightarrow Y^{\perp}$ come from different generalized eigenspaces where the first $\ell$ eigenvalues match. See Section 8 for how to compute this.

\section{Examples}

8(a). Zero-dimensional examples. Consider some cases of involutive tableaux with $\left(s_{1}, s_{2}, s_{3}\right)=(4,0,0)$.

$$
\left(\pi_{i}^{a}\right)=\left(\begin{array}{lll}
\pi_{1}^{1} & \pi_{2}^{1} & \pi_{3}^{1} \\
\pi_{1}^{2} & \pi_{2}^{2} & \pi_{3}^{2} \\
\pi_{1}^{3} & \pi_{2}^{3} & \pi_{3}^{3} \\
\pi_{1}^{4} & \pi_{2}^{4} & \pi_{3}^{4}
\end{array}\right)
$$

Or, in endovolutive block form:

$$
\left(\mathrm{B}_{i}^{\lambda}\right)=\left[\begin{array}{lll}
I_{4} & \mathrm{~B}_{2}^{1} & \mathrm{~B}_{3}^{1}
\end{array}\right]
$$

The characteristic ideal $\mathcal{M}$ will have degree $s_{\ell}=4$ and projective dimension $\ell-1=0$. That is, $\Xi$ will be 4 points, counted with multiplicity. The involutivity condition is $0=$ $\mathrm{B}_{2}^{1} \mathrm{~B}_{3}^{1}-\mathrm{B}_{3}^{1} \mathrm{~B}_{2}^{1}$ (all rows); that is, the matrices commute. Thus the matrices $\mathrm{B}_{2}^{1}$ and $\mathrm{B}_{3}^{1}$ must have compatible Jordan-block forms; they span a commutative algebra. In these examples, we will use four shades to emphasize the distinct generalized eigenspaces.

One possibility is that the matrices are diagonal with distinct Jordan blocks:

$$
A=\left\{\left(\begin{array}{lll}
\alpha_{1} & c_{1} \alpha_{1} & d_{1} \alpha_{1} \\
\alpha_{2} & c_{2} \alpha_{2} & d_{2} \alpha_{2} \\
\alpha_{3} & c_{3} \alpha_{3} & d_{3} \alpha_{3} \\
\alpha_{4} & c_{4} \alpha_{4} & d_{4} \alpha_{4}
\end{array}\right): \alpha_{a} \in \mathbb{C}\right\}
$$

In this case, the rank- 1 variety is

$$
\mathscr{C}=\left\{\left[\begin{array}{l}
1 \\
0 \\
0 \\
0
\end{array}\right] \otimes\left[1: c_{1}: d_{1}\right],\left[\begin{array}{l}
0 \\
1 \\
0 \\
0
\end{array}\right] \otimes\left[1: c_{2}: d_{2}\right],\left[\begin{array}{l}
0 \\
0 \\
1 \\
0
\end{array}\right] \otimes\left[1: c_{3}: d_{3}\right],\left[\begin{array}{l}
0 \\
0 \\
0 \\
1
\end{array}\right] \otimes\left[1: c_{4}: d_{4}\right]\right\}
$$

Each point $\xi \in \Xi$ has multiplicity 1 .

Another possibility is that they are diagonal, but there is a two-dimensional eigenspace.

$$
A=\left\{\left(\begin{array}{lll}
\alpha_{1} & c_{1} \alpha_{1} & d_{1} \alpha_{1} \\
\alpha_{2} & c_{1} \alpha_{2} & d_{1} \alpha_{2} \\
\alpha_{3} & c_{3} \alpha_{3} & d_{3} \alpha_{3} \\
\alpha_{4} & c_{4} \alpha_{4} & d_{4} \alpha_{4}
\end{array}\right): \alpha_{a} \in \mathbb{C}\right\}
$$

In this case, the rank-1 cone is

$$
\mathscr{C}=\left\{\left[\begin{array}{l}
* \\
* \\
0 \\
0
\end{array}\right] \otimes\left[1: c_{1}: d_{1}\right],\left[\begin{array}{l}
0 \\
0 \\
1 \\
0
\end{array}\right] \otimes\left[1: c_{3}: d_{3}\right],\left[\begin{array}{l}
0 \\
0 \\
0 \\
1
\end{array}\right] \otimes\left[1: c_{4}: d_{4}\right]\right\} .
$$


One point $\xi \in \Xi$ has multiplicity 2 ; in particular, the fiber ker $\sigma_{\xi}$ for $\xi=\left[1: c_{1}: d_{1}\right]$ should be seen as a $\mathbb{P}^{1}$. This is reflected clearly in 7.14 , because $\xi=\left[\xi_{1}: \xi_{2}: \xi_{3}\right]=\left[1: c_{1}: d_{1}\right]$ is a root of degree 2 for any $v$ :

$$
\begin{aligned}
0 & =\operatorname{det}\left(\xi_{1}\left(v^{2} \mathrm{~B}_{2}^{1}+v^{3} \mathrm{~B}_{3}^{1}\right)-\left(\xi_{2} v^{2}+\xi^{3} v_{3}\right) I\right) \\
& =\left|v^{2}\left(\begin{array}{cccc}
c_{1}-c_{1} & 0 & 0 & 0 \\
0 & c_{1}-c_{1} & 0 & 0 \\
0 & 0 & c_{3}-c_{1} & 0 \\
0 & 0 & 0 & c_{4}-c_{1}
\end{array}\right)+v^{3}\left(\begin{array}{cccc}
d_{1}-d_{1} & 0 & 0 & 0 \\
0 & d_{1}-d_{1} & 0 & 0 \\
0 & 0 & d_{3}-d_{1} & 0 \\
0 & 0 & 0 & d_{4}-d_{1}
\end{array}\right)\right| \\
& =v^{2}\left(c_{1}-c_{1}\right)^{2}\left(c_{3}-c_{1}\right)\left(c_{4}-c_{1}\right)+v^{3}\left(d_{1}-d_{1}\right)^{2}\left(d_{3}-d_{1}\right)\left(d_{4}-d_{1}\right) .
\end{aligned}
$$

Another possibility is that there is a $2 \times 2$ block:

$$
A=\left\{\left(\begin{array}{ccc}
\alpha_{1} & c_{1} \alpha_{1}+\alpha_{2} & d_{1} \alpha_{1}+\alpha_{2} \\
\alpha_{2} & c_{1} \alpha_{2} & d_{1} \alpha_{2} \\
\alpha_{3} & c_{3} \alpha_{3} & d_{3} \alpha_{3} \\
\alpha_{4} & c_{4} \alpha_{4} & d_{4} \alpha_{4}
\end{array}\right): \alpha_{a} \in \mathbb{C}\right\}
$$

In this case, the rank-1 cone is

$$
\mathscr{C}=\left\{\left[\begin{array}{l}
1 \\
0 \\
0 \\
0
\end{array}\right] \otimes\left[1: c_{1}: d_{1}\right],\left[\begin{array}{l}
0 \\
0 \\
1 \\
0
\end{array}\right] \otimes\left[1: c_{3}: d_{3}\right],\left[\begin{array}{l}
0 \\
0 \\
0 \\
1
\end{array}\right] \otimes\left[1: c_{4}: d_{4}\right]\right\} .
$$

Note that the fiber of $\mathscr{C}$ over $\Xi$ has dimension 1 in each case; however, the first point has multiplicity 2. We see that the dimension of the fiber is insufficient to measure the multiplicity of the scheme $\Xi$, because the incidence correspondence involves the ideal $\mathscr{R}$. We can see this because of the structure of the rank- 1 matrices: the upper $2 \times 2$ minors vanish if and only if $\alpha_{2} \alpha_{2}=0$, so the fiber ker $\sigma_{\xi}$ for $\xi=\left[1: c_{1}: d_{1}\right]$ should be seen as a $\mathbb{P}^{0}$ of degree 2 . This is reflected clearly in (7.14), because $\xi=\left[\xi_{1}: \xi_{2}: \xi_{3}\right]=\left[1: c_{1}: d_{1}\right]$ is a root of degree 2 for any $v$ :

$$
\begin{aligned}
0 & =\operatorname{det}\left(\xi_{1}\left(v^{2} \mathrm{~B}_{2}^{1}+v^{3} \mathrm{~B}_{3}^{1}\right)-\left(\xi_{2} v^{2}+\xi^{3} v_{3}\right) I\right) \\
& =v^{2}\left(\begin{array}{cccc}
c_{1}-c_{1} & 1 & 0 & 0 \\
0 & c_{1}-c_{1} & 0 & 0 \\
0 & 0 & c_{3}-c_{1} & 0 \\
0 & 0 & 0 & c_{4}-c_{1}
\end{array}\right)+v^{3}\left(\begin{array}{cccc}
d_{1}-d_{1} & 1 & 0 & 0 \\
0 & d_{1}-d_{1} & 0 & 0 \\
0 & 0 & d_{3}-d_{1} & 0 \\
0 & 0 & 0 & d_{4}-d_{1}
\end{array}\right) \mid \\
& =v^{2}\left(c_{1}-c_{1}\right)^{2}\left(c_{3}-c_{1}\right)\left(c_{4}-c_{1}\right)+v^{3}\left(d_{1}-d_{1}\right)^{2}\left(d_{3}-d_{1}\right)\left(d_{4}-d_{1}\right) .
\end{aligned}
$$

Finally, consider the case where both types of multiplicity occur. For example,

$$
A=\left\{\left(\begin{array}{ccc}
\alpha_{1} & c_{1} \alpha_{1}+\alpha_{2} & d_{1} \alpha_{1}+\alpha_{2} \\
\alpha_{2} & c_{1} \alpha_{2} & d_{1} \alpha_{2} \\
\alpha_{3} & c_{1} \alpha_{3} & d_{1} \alpha_{3} \\
\alpha_{4} & c_{4} \alpha_{4} & d_{4} \alpha_{4}
\end{array}\right): \alpha_{a} \in \mathbb{C}\right\}
$$


In this case, the rank- 1 cone is

$$
\mathscr{C}=\left\{\left[\begin{array}{l}
* \\
0 \\
* \\
0
\end{array}\right] \otimes\left[1: c_{1}: d_{1}\right],\left[\begin{array}{l}
0 \\
0 \\
0 \\
1
\end{array}\right] \otimes\left[1: c_{4}: d_{4}\right]\right\} .
$$

The scheme structure of $\Xi$ is apparent here. The point $\xi=\left[1: c_{1}: d_{1}\right]$ appears in two components, which correspond to the factorization of

$$
\begin{aligned}
0 & =\operatorname{det}\left(\xi_{1}\left(v^{2} \mathrm{~B}_{2}^{1}+v^{3} \mathrm{~B}_{3}^{1}\right)-\left(\xi_{2} v^{2}+\xi^{3} v_{3}\right) I\right) \\
& =\left|v^{2}\left(\begin{array}{cccc}
c_{1}-c_{1} & 1 & 0 & 0 \\
0 & c_{1}-c_{1} & 0 & 0 \\
0 & 0 & c_{1}-c_{1} & 0 \\
0 & 0 & 0 & c_{4}-c_{1}
\end{array}\right)+v^{3}\left(\begin{array}{cccc}
d_{1}-d_{1} & 1 & 0 & 0 \\
0 & d_{1}-d_{1} & 0 & 0 \\
0 & 0 & d_{1}-d_{1} & 0 \\
0 & 0 & 0 & d_{4}-d_{1}
\end{array}\right)\right| \\
& =v^{2}\left(c_{1}-c_{1}\right)^{2}\left(c_{1}-c_{1}\right)\left(c_{4}-c_{1}\right)+v^{3}\left(d_{1}-d_{1}\right)^{2}\left(d_{1}-d_{1}\right)\left(d_{4}-d_{1}\right) .
\end{aligned}
$$

From the perspective of $\mathscr{C}$, these components correspond to the rank-1 matrices

$$
\left(\begin{array}{ccc}
\alpha_{1} & c_{1} \alpha_{1} & d_{1} \alpha_{1} \\
0 & 0 & 0 \\
\alpha_{3} & c_{1} \alpha_{3} & d_{1} \alpha_{3} \\
0 & 0 & 0
\end{array}\right)
$$

The fiber should be seen as two components, a $\mathbb{P}^{1}$ and a $\mathbb{P}^{0}$. Overall, this point has multiplicity 3 .

8.15. REMARK. For readers interested in hydrodynamic integrability criteria, take a moment to compute the secant varieties $\operatorname{Sec}_{k}(\mathscr{C})$ and $\operatorname{Sec}_{k}(\Xi), k=2,3$, in each of these cases. The secant variety is all linear combinations of $k$ points from the given variety. One can consider both the embedded secant variety within $A$ and $V^{*}$, respectively, as well as the Grassmannian secant variety within $\operatorname{Gr}_{k}(A)$ and $\operatorname{Gr}_{k}\left(V^{*}\right)$, respectively. Note that hyperbolic systems of conservation laws have $s_{1}=n$ and take the non-degenerate diagonal form of the first example, over $\mathbb{R}$.

8(b). One-dimensional examples. Consider an involutive tableau with $\left(s_{1}, s_{2}, s_{3}\right)=$ $(2,1,0)$.

$$
\left(\pi_{i}^{a}\right)=\left(\begin{array}{lll}
\pi_{1}^{1} & \pi_{2}^{1} & \pi_{3}^{1} \\
\pi_{1}^{2} & \pi_{2}^{2} & \pi_{3}^{2}
\end{array}\right) .
$$

Or, in endovolutive block form,

$$
\left(\mathrm{B}_{i}^{\lambda}\right)=\left[\begin{array}{ccc}
\left(\begin{array}{ll}
1 & 0 \\
0 & 1
\end{array}\right) & \left(\begin{array}{cc}
0 & 0 \\
x_{0} & x_{1}
\end{array}\right) & \left(\begin{array}{ll}
x_{2} & x_{3} \\
x_{4} & x_{5}
\end{array}\right) \\
\left(\begin{array}{ll}
0 & 0 \\
0 & 0
\end{array}\right) & \left(\begin{array}{ll}
1 & 0 \\
0 & 0
\end{array}\right) & \left(\begin{array}{cc}
x_{6} & 0 \\
0 & 0
\end{array}\right)
\end{array}\right] .
$$

The characteristic ideal $\mathcal{M}$ will have degree $s_{\ell}=1$ and projective dimension $\ell-1=1$. That is, $\Xi$ will be a single curve. 
For the sake of concreteness, let us assume that the coefficients are:

$$
\left(\mathrm{B}_{i}^{\lambda}\right)=\left[\begin{array}{lll}
\left(\begin{array}{ll}
1 & 0 \\
0 & 1
\end{array}\right) & \left(\begin{array}{ll}
0 & 0 \\
\frac{1}{9} & 0
\end{array}\right) & \left(\begin{array}{ll}
5 & 0 \\
1 & 5
\end{array}\right) \\
\left(\begin{array}{ll}
0 & 0 \\
0 & 0
\end{array}\right) & \left(\begin{array}{ll}
1 & 0 \\
0 & 0
\end{array}\right) & \left(\begin{array}{ll}
9 & 0 \\
0 & 0
\end{array}\right)
\end{array}\right]
$$

so that

$$
\left(\pi_{i}^{a}\right)=\left(\begin{array}{ccc}
\alpha_{0} & \alpha_{2} & 5 \alpha_{0}+9 \alpha_{2} \\
\alpha_{1} & \frac{1}{9} \alpha_{0} & \alpha_{0}+5 \alpha_{1}
\end{array}\right)
$$

The rank- 1 ideal is just $\alpha_{0} \alpha_{0}-9 \alpha_{1} \alpha_{2}=0$. Write a generic element of $\mathscr{C}$ as $\left[\alpha_{0}: \alpha_{1}: \alpha_{2}\right]=$ $\left[3 \tau: 1: \tau^{2}\right]$, like so:

$$
\left(\begin{array}{ccc}
3 \tau & \tau^{2} & 15 \tau+9 \tau^{2} \\
1 & \frac{1}{3} \tau & 5+3 \tau
\end{array}\right)
$$

Thus, a generic element of $\xi$ is of the form $\xi=[3: \tau: 15+9 \tau]$ with fiber $\left[\begin{array}{c}3 \tau \\ 1\end{array}\right]$.

By using 7.14, the characteristic scheme of $\xi=\left[3: \xi_{2}: \xi_{3}\right]$ is generated by $0=$ $\operatorname{det}\left(\xi_{1} v^{3} \mathrm{~B}_{3}^{1}+\xi_{2} v^{3} \mathrm{~B}_{3}^{2}-\xi_{3} v^{3} I_{2}\right)$, restricted to the space $\mathbf{W}^{1}(\xi) \subset W$, which is one-dimensional. Write $\tau$ for $\xi_{2}$; so we are trying to find $\xi=\left[3: \tau: \xi_{3}\right]$ over $\varphi=[3: \tau: 0]$ as in Lemma 7.4 The space $\mathbf{W}^{1}(\varphi)$ is the space spanned by $\left[\begin{array}{c}3 \tau \\ 1\end{array}\right]$. Hence, the single linear sheet of the characteristic variety over $[3: \tau: 0]$ is given by $[3: \tau: 15+9 \tau]$.

8(c). One-dimensional exercise. Now is the time go back and re-read the example (1.4) and see how it fits into Sections 6(c) and 5(a) The wave-equation example offers a single $\mathbb{P}^{1}$ whose fiber is also a $\mathbb{P}^{1}$. By choosing appropriate coefficients, you should be able to produce examples with $\left(s_{1}, s_{2}, s_{3}\right)=(3,2,0)$ with various other components and multiplicities.

In principle, you can choose any Cartan characters, and choose coefficients subject to Theorem 5.4 to build examples in this way. See the Sage code at https://bitbucket. org/curieux/symbol_sage, which can generate and analyze any such example (given sufficient memory).

9. Results of Guillemin and Quillen. As in the analogy Section 1(b) normal forms often reveal shortcuts to other advanced ideas.

Guillemin's proof of Theorem 7.6 made use of two results derived from Quillen's thesis Qui64. In this section, we see how these results become easier by using Theorem 5.4 (Note that Theorem 5.4 and Theorem 7.6 are not equivalent. Theorem 5.4 is strictly stronger; it is easy to construct endovolutive tableaux that satisfy the conclusion of (7.7) but are not involutive. See Smi15.)

Recall the Spencer cohomology groups from Section 4(b). For any $\varphi \in V^{*}$, wedging by $\varphi$ gives a map $W \otimes \wedge^{k} V^{*} \rightarrow W \otimes \wedge^{k+1} V^{*}$. This induces a map on the quotient spaces, $H^{k}(A) \rightarrow H^{k+1}(A)$. 
9.1. TheOrem (Quillen's Exactness Theorem). Suppose A is an involutive tableau, and that $\varphi \notin \Xi_{A}$. Then the sequence of maps by $\wedge \varphi$,

$$
0 \rightarrow A \rightarrow H^{1}(A) \rightarrow H^{2}(A) \rightarrow \ldots \rightarrow H^{n}(A) \rightarrow 0,
$$

is exact.

In Qui64, this theorem is proven using enormous commutative diagrams. In our context, with Theorem 5.4 in hand, we can prove an easy version of Quillen's result, in the form of Lemma 9.3 Lemma 9.3 is a consequence of Corollary 9.2 , which for us is an easy corollary of Theorem 5.4. This corollary is called Theorem A in [Gui68, where it was proved using a large diagram chase using Quillen's exactness theorem, Theorem 9.1 . 9.2. Corollary (Quillen, Guillemin). Consider the subspace $U=\left\langle u_{1}, \ldots, u_{\ell}\right\rangle \subset V$ for a generic basis $\left(u_{i}\right)$ of $V$, as in (1.17). If $A$ is involutive, then $\left.A\right|_{U}$ is involutive, and the natural map between prolongations $A^{(1)} \rightarrow\left(\left.A\right|_{U}\right)^{(1)}$ is bijective.

Proof. The first part is an immediate consequence of Theorem 5.4 as the quadratic condition still holds if the range of indices $\lambda, \mu, i, j$ is truncated at $\ell$ (or greater). In particular, the generators $\left(\pi_{\lambda}^{a}\right)_{a \leq s_{\lambda}}$ of $A$ are preserved.

The second part is similarly immediate, using the proof of Theorem 5.4 given in Smi15]: the contact relation $\pi_{\mu}^{a}=Z_{\mu, i}^{a} u^{i}$ for $a \leq s_{\lambda}$ gives coordinates $Z_{\mu, i}^{a}$ to the prolongation $A^{(1)} \subset A \otimes V^{*}$, and the $s_{1}+2 s_{2}+\ldots+\ell s_{\ell}$ independent generators are precisely those $Z_{\mu, \lambda}^{a}$ with $a \leq s_{\mu}$ and $\lambda \leq \mu \leq \ell$. Since they involve no indices $i>\ell$, these generators remain independent when the range of indices is truncated at $\ell$.

Now we come to our simplified version of Theorem 9.1. Compare Lemma 9.3 to the exact sequence $(3.4)_{2}$ in Gui68.

9.3. Lemma. Recall that $U^{\perp}$ is a complement to $Y^{\perp} \subset V^{*}$, so that $V^{*}=Y^{\perp} \oplus U^{\perp}$ as in (1.17) and (1.18). For $A$ involutive, the sequence

$$
0 \rightarrow W \otimes S^{2} U^{\perp} \rightarrow H^{1} \otimes U^{\perp} \stackrel{\delta}{\rightarrow} H^{2}
$$

is exact.

Proof. This proof is just an explicit description of the maps in a basis and an application of Corollary 9.2 Let $\left(u^{i}\right)$ be a basis for $V^{*}$ such that $\left(u^{\lambda}\right)$ is a basis for $Y^{\perp}$ and $\left(u^{\varrho}\right)$ is a basis for $U^{\perp}$, using the index convention 1.8 from Section 1 .

The sequence makes sense because we can split the Spencer sequence (4.15) as $W \otimes V^{*}=A \oplus H^{1}$ by identifying the space $H^{1}$ with $\left\{\sum_{a>s_{i}} \pi_{i}^{a}\left(z_{a} \otimes u^{i}\right)\right\} \subset W \otimes V^{*}$, which is the space spanned by the unshaded entries in Fig. 11. With this identification, two elements $\sum_{a>s_{i}} \pi_{i}^{a}\left(z_{a} \otimes u^{i}\right)$ and $\sum_{a>s_{i}} \hat{\pi}_{i}^{a}\left(z_{a} \otimes u^{i}\right)$ of $W \otimes V^{*}$ are equivalent in $H^{1}$ if and only if $\pi_{i}^{a}-\hat{\pi}_{i}^{a}=\sum_{b \leq s_{\lambda}} B_{i, b}^{a, \lambda} z_{i}^{b}$ for some $\left\{z_{i}^{a}: a \leq s_{i}\right\}$, the shaded entries in Fig. 1 . In other words, the projection $W \otimes V^{*} \rightarrow H^{1}$ is defined by 1.10 , and the projection $W \otimes V^{*} \rightarrow A$ is defined by the projection onto the shaded generator components in Fig. 1 . those $\pi_{\lambda}^{a}$ with $a \leq s_{\lambda}$.

Since $s_{\varrho}=0$ for all $\varrho>\ell$, the inclusion $W \otimes U^{\perp} \subset W \otimes V^{*}$ is an inclusion $W \otimes U^{\perp} \subset H^{1}$. Hence, the inclusion is understood as

$$
W \otimes S^{2} U^{\perp} \subset\left(W \otimes U^{\perp}\right) \otimes U^{\perp} \subset H^{1} \otimes U^{\perp} .
$$


An element of $H^{1} \otimes U^{\perp}$ is written in $W \otimes V^{*} \otimes U^{\perp}$ as

$$
P=\sum_{a>s_{\lambda}} P_{\lambda, \varsigma}^{a}\left(z_{a} \otimes u^{\lambda} \otimes u^{\varsigma}\right)+\sum_{a>0} P_{\varrho, \varsigma}^{a}\left(z_{a} \otimes u^{\varrho} \otimes u^{\varsigma}\right) .
$$

The image $\delta\left(H^{1} \otimes U^{\perp}\right)$ in $H^{2}$ is

$$
\delta\left(H^{1} \otimes V^{*}\right) \subset \delta\left(W \otimes V^{*} \otimes V^{*}\right) \subset W \otimes \wedge^{2} V^{*},
$$

so $\delta P \in W \otimes \wedge^{2} V^{*}$ is of the form

$$
\delta P=\sum_{a>s_{\lambda}} P_{\lambda, \varsigma}^{a}\left(z_{a} \otimes u^{\lambda} \wedge u^{\varsigma}\right)+\sum_{a>0} \frac{1}{2}\left(P_{\varrho, \varsigma}^{a}-P_{\varsigma, \varrho}^{a}\right)\left(z_{a} \otimes u^{\varrho} \wedge u^{\varsigma}\right) .
$$

Recall that $H^{2}=\frac{W \otimes \wedge^{2} V^{*}}{\delta_{\sigma}\left(A \otimes V^{*}\right)}$. So, $\delta P \equiv 0$ in $H^{2}$ if and only if there is some $T \in A \otimes V^{*}$ such that $\delta_{\sigma}(T)=\delta(P)$ in $W \otimes \wedge^{2} V^{*}$. Looking at (9.7), it is apparent that such $T$ must have $\delta_{\sigma}\left(\left.T\right|_{U}\right)=0$, as $\delta(P)$ has no $Y^{\perp} \wedge Y^{\perp}$ terms. By involutivity and Corollary 9.2 , we consider the involutive tableau

$$
\left.0 \rightarrow A\right|_{U} \rightarrow W \otimes Y^{\perp} \stackrel{\left.\sigma\right|_{U}}{\rightarrow} H_{U}^{1} \rightarrow 0
$$

with prolongation

$$
\left.0 \rightarrow\left(\left.A\right|_{U}\right)^{(1)} \rightarrow A\right|_{U} \otimes Y^{\perp} \stackrel{\left.\delta_{\sigma}\right|_{U}}{\rightarrow} W \otimes \wedge^{2} Y^{\perp} \rightarrow H_{U}^{2} \rightarrow 0 .
$$

Therefore, $\left.\left.T\right|_{U} \in A\right|_{U} \otimes Y^{\perp}$ lies in the kernel of $\left.\delta_{\sigma}\right|_{U}$, so $\left.T\right|_{U} \in\left(\left.A\right|_{U}\right)^{(1)}$. Therefore, Corollary 9.2 tells us $T \in A^{(1)}$. That is, $\delta(P) \equiv 0 \in H^{2}$ if and only if $\delta(P)=\delta_{\sigma}(T)=0$.

Therefore, $\delta(P) \equiv 0 \in H^{2}$ if and only if $P_{\lambda, \varsigma}^{a}=0$ and $P_{\varrho, \varsigma}^{a}=P_{\varsigma, \varrho}^{a}$ on these index ranges. This occurs if and only if $P=P_{\varrho, \varsigma}^{a}\left(z_{a} \otimes u^{\varrho} \otimes u^{\varsigma}\right)$, meaning $P \in W \otimes S^{2} U^{\perp}$.

We are ready to prove Theorem 7.6. The structure of the proof is identical to the original proof in Gui68.

Proof of Theorem 7.6. Suppose that $w \in \mathbf{W}^{1}(\varphi)$, so that $\pi=\mathrm{B}(\varphi)(\cdot) w=w \otimes \varphi+J$ for some $J \in W \otimes U^{\perp}$ with $J_{\varrho}=J_{\varrho}^{a} z_{a} \in \mathbf{W}^{-}(\varphi)$ for all $\varrho$. First, we must show that the span of the columns $J_{\varrho}$ of $J$ lies in $\mathbf{W}^{1}(\varphi)$.

Consider the element $-J \otimes \varphi=-J_{\varrho}^{a} \varphi_{\lambda}\left(z_{a} \otimes u^{\lambda} \otimes u^{\varrho}\right) \in H^{1} \otimes U^{\perp}$. Because $z \otimes \varphi+J \in A$, it must be that $z \otimes \varphi \otimes \varphi \in W \otimes V^{*} \otimes V^{*}$ represents the same point in $H^{1} \otimes U^{\perp}$. So, we can compute

$$
-J_{\varrho}^{a} \varphi_{\lambda}\left(z_{a} \otimes u^{\lambda} \wedge u^{\varrho}\right) \equiv z \otimes \varphi \wedge \varphi=0 \in H^{2} .
$$

By Corollary 9.2 there exists $Q=Q_{\varrho, \varsigma}^{a}\left(z_{a} \otimes u^{\varsigma} \otimes u^{\varrho}\right) \in W \otimes S^{2} U^{\perp}$ such that $-J \otimes \varphi-Q \in$ $A \otimes U^{\perp}$. That is, writing $Q_{\varrho}=Q_{\varrho, \varsigma}^{a}\left(z_{a} \otimes u^{\varsigma}\right) \in W \otimes Y^{\perp}$, we have $J_{\varrho} \otimes \varphi+Q_{\varrho} \in A$ for all $\varrho$, meaning $J_{\varrho} \in \mathbf{W}^{1}(\varphi)$ for all $\varrho$. Therefore, for any $v \in V$, we have $\operatorname{B}(\varphi)(v) z=$ $\varphi(v) z+J(v) \in \mathbf{W}^{1}(\varphi)$.

Now, mapping again, $\mathrm{B}(\varphi)(\cdot) J_{\varrho}=J_{\varrho} \otimes \varphi+Q_{\varrho}$, so $\mathrm{B}(\varphi)\left(u_{\varsigma}\right) J_{\varrho}=Q_{\varrho, \varsigma}$, which is already known to be symmetric in $\varrho, \varsigma$. Therefore,

$$
\begin{aligned}
\mathrm{B}(\varphi)(\tilde{v}) \mathrm{B}(\varphi)(v) z & =\mathrm{B}(\varphi)(\tilde{v})(\varphi(v) z+J(v)) \\
& =\varphi(v) \mathrm{B}(\varphi)(\tilde{v}) z+u^{\varrho}(v) \mathrm{B}(\varphi)(\tilde{v}) J_{\varrho} \\
& =\varphi(v)(\varphi(\tilde{v}) z+J(\tilde{v}))+u^{\varrho}(v)\left(\varphi(\tilde{v}) J_{\varrho}+Q_{\varrho}(\tilde{v})\right) \\
& =\varphi(v) \varphi(\tilde{v}) z+\varphi(v) J(\tilde{v})+\varphi(\tilde{v}) J(v)+Q(v, \tilde{v}) .
\end{aligned}
$$

This is symmetric in $v, \tilde{v}$, giving the commutativity condition (7.7) 
It is interesting to see the inversion of logic that happened here. In the original literature, the overall implications are

$$
9.1 \rightarrow 9.3 \rightarrow 9.2 \rightarrow 7.6
$$

But, the arguments here give the overall implications

$$
[5.4 \rightarrow 9.2 \rightarrow 9.3 \rightarrow 7.6
$$

However, we can write a shorter proof of Theorem 7.6 that relies Theorem 5.4 more directly, avoiding the general results of Quillen. For motivation, consider the following trivial corollary of Theorem 5.4 that is obtained by setting $\lambda=\mu$.

9.12. Corollary. Suppose an involutive tableau is given in a generic, endovolutive basis as in (1.14), so that Theorem 5.4 holds. Then $\mathrm{B}\left(u^{\lambda}\right)(v)$ is an endomorphism of $\mathbf{W}^{-}\left(u^{\lambda}\right)$ such that for all $v, \tilde{v} \in Y$,

$$
\left[\mathrm{B}\left(u^{\lambda}\right)(v), \mathrm{B}\left(u^{\lambda}\right)(\tilde{v})\right]=0 .
$$

Alternate Proof of Theorem 7.6. Fix $\varphi \in Y^{\perp}$, and suppose that $w \in \mathbf{W}^{1}(\varphi)$. We must verify that all maps $\mathrm{B}(\varphi)(v)$ preserve $\mathbf{W}^{1}(\varphi)$ and that they commute. Note that the definition of $\mathbf{W}^{1}(\varphi)$ in equation 1.24 depends on the choice of subspace $Y^{\perp}$ but not on its basis, so we may verify these conditions using any basis we like.

First a trivial case: if it happens that $\varphi \in \Xi \cap Y^{\perp}$, then $\mathrm{B}(\varphi)(v) w=\varphi(v) w \in \mathbf{W}^{1}(\varphi)$ is a rescaling, and it is immediate that $[\mathrm{B}(\varphi)(v), \mathrm{B}(\varphi)(\tilde{v})]=0$.

Otherwise, we have $\varphi \notin \Xi$. Then we may choose a generic basis of $V^{*}$ in which $\varphi=u^{1}$. Moreover, we may use that basis to construct an endovolutive basis of $W$. By Corollary 9.12, it suffices to prove in this basis that $\mathbf{W}^{1}\left(u^{1}\right)$ is preserved by every $\mathrm{B}\left(u^{1}\right)(v)$. Write $\mathrm{B}(\varphi)(\cdot) w=w \otimes u^{1}+J$, and examine 1.22 on a column $J_{\varrho}$ of $J$. For each $\mu=1, \ldots, \ell$, we must verify

$$
0=\left(\mathrm{B}_{\mu}^{1}-\delta_{\mu}^{1} I\right) J_{\varrho}=\left(\mathrm{B}_{\mu}^{1}-\delta_{\mu}^{1} I\right) \mathrm{B}_{\varrho}^{1} w=\left(\mathrm{B}_{\mu}^{1} \mathrm{~B}_{\varrho}^{1}-\delta_{\mu}^{1} \mathrm{~B}_{\varrho}^{1}\right) w .
$$

If $\mu=1$, then this is immediate, since $\mathrm{B}_{1}^{1}=I_{s_{1}}$.

If $\mu \neq 1$, then we are verifying $0=\left(\mathrm{B}_{\mu}^{1} \mathrm{~B}_{\varrho}^{1}-0\right) w$. Note that $\mathrm{B}_{\mu}^{1} w=0$, since $\mathrm{B}(\varphi)(\cdot) w=$ $w \otimes \varphi+J=w \otimes u^{1}+J$. Moreover, by Theorem 5.4. we have

$$
0=\left(\mathrm{B}_{\mu}^{1} \mathrm{~B}_{\varrho}^{1}-\mathrm{B}_{\varrho}^{1} \mathrm{~B}_{\mu}^{1}\right)_{b}^{a} w^{b}=\left(\mathrm{B}_{\mu}^{1} \mathrm{~B}_{\varrho}^{1}\right)_{b}^{a} w^{b}
$$

for $a>s_{\mu}$. Therefore, $\mathrm{B}_{\mu}^{1} \mathrm{~B}_{\varrho}^{1}$ lies in $\mathbf{W}^{-}(\mu)$. On the other hand, note that the output of $\mathrm{B}_{\mu}^{1}$ lies in $\mathbf{W}_{\mu}^{+}$by the construction of the maps $\mathrm{B}_{\mu}^{\lambda}$ from the reduced symbol in Section 1(c). Combining these, we see that $\mathrm{B}_{\mu}^{1} \mathrm{~B}_{\varrho}^{1} w$ lies in $\mathbf{W}_{\mu}^{-} \cap \mathbf{W}_{\mu}^{+}=0$.

Hence, the space $\mathbf{W}^{1}(\varphi)$ is preserved by $\mathrm{B}(\varphi)(v)$ for all $v$. By Corollary 9.12 they commute.

On the theoretical side, it would be interesting to see how many of the hard classical theorems in the subject can be re-proven with elementary techniques. Specifically, the proof of Lemma 9.3 suggests an elementary proof of Quillen's exactness theorem. The other hard theorem is the integrability of the characteristic variety, and a proof of that theorem using Guillemin's original formulation is the subject of GQS70. That result was applied immediately to study primitive Lie pseudogroups. 
10. Prolongation. How does the characteristic scheme change under prolongation? The short answer is that it does not! This does not depend on endovolutivity or involutivity.

Recall that $A^{(1)}$ is a tableau within $A \otimes V^{*}$. An element of $A^{(1)}$ is $P \in A \otimes V^{*}$. Using any bases for $V, W, A$, we may write $P$ as $P_{i, j}^{a} z_{a} \otimes u^{i} \otimes u^{j}$, with the additional condition that $P_{i, j}^{a}=P_{j, i}^{a}$ from 4.9 . Let $\mathscr{C}^{(1)}$ denote the rank-1 elements of $A^{(1)}$, and let $\Xi^{(1)}$ denote its projection to $V^{*}$, as in Section 6(b)

10.1. TheOREM. If $\pi \otimes \xi \in \mathscr{C}^{(1)}$, then $\pi=w \otimes \xi \in \mathscr{C}$ for some $w \in \operatorname{ker} \sigma_{\xi}$. Conversely, if $w \otimes \xi \in \mathscr{C}$, then $(w \otimes \xi) \otimes \xi \in \mathscr{C}^{(1)}$. In particular, $\Xi \cong \Xi^{(1)}$ as schemes.

Proof. Suppose that $\pi \otimes \xi \in \mathscr{C}^{(1)}$ for some $\pi \in A$ and $\xi \in V^{*}$. That is, $P \in A^{(1)}$ and $P=\pi \otimes \xi$, so $P_{i, j}^{a}=\pi_{i}^{a} \xi_{j}$, and $\pi_{i}^{a} \xi_{j}=\pi_{j}^{a} \xi_{i}$ for all $a, i, j$.

Let $\underline{\lambda}$ be the minimum index such that $\xi_{\underline{\lambda}} \neq 0$. Then $\pi_{\lambda}^{a} \xi_{i}=\pi_{i}^{a} \xi_{\lambda}$, so column $i$ of $\left(\pi_{i}^{a}\right)$ is a multiple - namely $\xi_{i} / \xi_{\underline{\lambda}}$ - of column $\underline{\lambda}$ for all $i$. Therefore, $\left(\pi_{i}^{a}\right)$ is rank-1, and there is some $w$ with $\pi=w \otimes \xi$. The converse is immediate.

10.2. REMARK. Theorem 10.1 is used sometimes as a method for computing the characteristic variety, as follows: Given a tableau $\left(\pi_{i}^{a}\right)$ whose entries might depend on $e \in M^{(1)}$, consider $\left(\xi_{i}\right) \mapsto\left(\pi_{i}^{a} \xi_{j}-\pi_{j}^{a} \xi_{i}\right)$ as a map $V^{*} \rightarrow W \otimes \wedge^{2} V^{*}$; that is, a map from $\mathbb{C}^{n}$ to

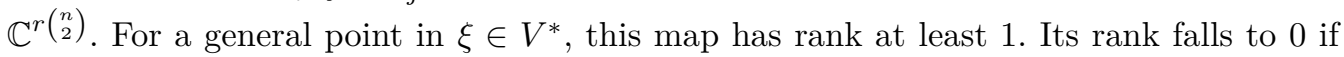
and only if $\xi \in \Xi$. But, this method is inefficient. If you have $\left(\pi_{i}^{a}\right)$ in hand and want to compute $2 \times 2$ minors of something, you would save ink by computing the $2 \times 2$ minors of $\left(\pi_{i}^{a}\right)$ itself to find $\mathscr{C}$.

11. Characteristic sheaf. For a single endomorphism, the characteristic polynomial and the Jordan block decomposition of generalized eigenspaces together reveal all of the information that is independent of coordinates.

The ultimate conclusion of the preceding sections is that, for an abstract tableau $A$, the characteristic sheaf $\mathscr{M}$ knows the dimensions $n, r,\left(s_{1}, \ldots, s_{n}\right)$, as well as all of the dimensions and relationships among the mutual eigenspaces of the various symbol maps. The rank-1 cone $\mathscr{C}$ knows the algebraic relationships among the sequences of eigenvalues (which we call $\xi$ ), and it also knows on which subspaces the symbol maps commute and on which fail to commute. In summary, $\mathscr{M}$ and $\mathscr{C}$ together know everything about an abstract tableau $A$ that is independent of coordinates ${ }^{23}$ Moreover, they are invariant under prolongation!

If the abstract tableau $A$ is a smooth projective bundle, then this applies to involutive Kähler-regular exterior differential systems in the smooth category.

If this formal perspective is appealing, then one might as well dispense with tableaux, symbols, Grassmann bundles, and differential ideals, and instead study the sheaf $\mathscr{M}$ directly, with modern algebraic tools such as [Eis05]. Consider $\mathscr{M}$ as an ideal in

$$
C^{\infty}\left(M^{(1)}\right)\left[u_{1}, \ldots, u_{n}\right]
$$

\footnotetext{
${ }^{23}$ We revealed this fact using special bases, but as with traditional Jordan normal form, there is an abstract structure independent of basis that is easiest to see by building an adapted basis.
} 
and consider its free resolution. The Hilbert syzygy theorem states that there is a finite free resolution that is characterized by its Hilbert polynomial $h_{\mathscr{M}}(d)$. Of course, Theorem 7.11 is reading the leading term of $h_{\mathscr{M}}(d)$ !

One might ask how the involutivity of $A$ can be detected as an algebraic property of $\mathscr{M}$. The answer is tied to Castelnuovo-Mumford regularity, which measures the growth of the Hilbert polynomial. This computation is equivalent to the Cartan characters in Cartan's test!

While it is not necessarily a useful computational tool versus differential forms or tableaux, this perspective allows a broader view of the techniques in PDE analysis, and it suggests that future progress in the field will emphasize on invariant algebraic techniques.

For more on this perspective, see [Mal03, $\mathrm{BCG}^{+} 90$, Chapter VIII], and the notes by Mark Green from the 2013 conference New Directions in Exterior Differential Systems in Estes Park, Colorado, which are based on the perspective in CGG09.

\section{Part IV. Eikonal systems}

In Part III we studied the characteristic scheme defined over $M^{(1)} \subset \operatorname{Gr}_{n}(T M)$. In this part, we turn our attention to the characteristic scheme as pulled back to an integral manifold $\iota: N \rightarrow M$. This is where the meaning of $\Xi$ as "directions with an ambiguous initial value problem" has clear implications for the internal structure of solutions of a differential equation, as the eikonal system yields intrinsic foliations of integral manifolds $N$.

12. General eikonal systems. First, let us consider the general notion of "eikonal equations" of a projective variety, without specific regard to the characteristic variety.

Consider a smooth manifold $N$ of dimension $n$. Here are three ways to produce a smooth local hypersurface $H \subset N$.

(i) The implicit function theorem says that a smooth hypersurface $H \subset N$ is defined locally by a smooth function $f: N \rightarrow \mathbb{R}$, where $T_{x} H=\operatorname{ker} \mathrm{d} f$ for all $x \in H$.

(ii) By the Frobenius theorem, this is equivalent to having a local smooth section $\varphi$ of $T^{*} N=\Omega^{1}(N)$ such that $\mathrm{d} \varphi \equiv 0 \bmod \varphi$, for then $\varphi$ is a rescaling of some $\mathrm{d} f$.

(iii) We can also look at the Frobenius theorem from the perspective of Cartan-Kähler theory ${ }^{24}$ as in Theorem 5.3 To make a local function $f: N \rightarrow \mathbb{R}$ or a local section $\varphi$ of $T^{*} N$, consider the jet space $\mathbb{J}^{1}(N, \mathbb{R})$, which is isomorphic to the bundle $T^{*} N \times \mathbb{R}$. Jet space is an open neighborhood (or local linearization) of $\operatorname{Gr}_{n}(N \times \mathbb{R})$ equipped with local coordinates $\left(x^{i}, p_{i}, y\right)=\left(x^{1}, \ldots, x^{n}, p_{1}, \ldots, p_{n}, y\right)$ and a contact system $\mathcal{J}$ generated by $\Upsilon=\mathrm{d} y-p_{i} \mathrm{~d} x^{i}$ and $\mathrm{d} \Upsilon$, as in Section 3(a). In these local coordinates, set the independence condition $\boldsymbol{\omega}=\mathrm{d} x^{1} \wedge \ldots \wedge \mathrm{d} x^{n} \neq 0$. Any $n$-dimensional integral manifold of the exterior differential system $\left(T^{*} N \times \mathbb{R}, \mathcal{J}, \boldsymbol{\omega}\right)$ corresponds to a function $y=f\left(x^{1}, \ldots, x^{n}\right)$ with $p_{i}=\frac{\partial f}{\partial x^{i}}$, so we may take $\varphi=\mathrm{d} f=\frac{\partial f}{\partial x^{i}} \mathrm{~d} x^{i}$. It is easy to

\footnotetext{
${ }^{24}$ Although Theorem 5.3 applies as stated only in the analytic category, it can be extended to the smooth category in this case. This sort of extension is explored in Section 14
} 
see that this exterior differential system has no torsion and has a Kähler-regular tableau with Cartan characters $s_{1}=s_{2}=\ldots=s_{n}=1$. That is, integral manifolds are parametrized by 1 function of $n$ variables (hardly a surprise).

Now, consider a projective subbundle $\Sigma_{N} \subset \mathbb{P} T^{*} N$, meaning it is defined smoothly by homogeneous functions in the local fiber variables $\left(p_{i}\right)$ of $T^{*} N$. We want a test that tells us whether there exist hypersurfaces $H$ for which $\mathrm{d} f \in \Sigma_{N}$ everywhere. Specifically, we want a theorem like the following.

12.1. TheOREM. Suppose that the eikonal system (defined below) of $\Sigma_{N}$ is involutive. Then for any smooth point $[\varphi] \in\left(\Sigma_{N}\right)_{x}$, there is a smooth hypersurface $H \subset N$ such that $\left(T_{x} H\right)^{\perp}=[\varphi]$ and such that $\left(T_{\tilde{x}} H\right)^{\perp}$ lies in the smooth locus of $\left(\Sigma_{N}\right)_{\tilde{x}}$ for all $\tilde{x} \in H$.

Because the hypersurface $H$ and the 1-form $\varphi$ are not chosen a priori, this condition is difficult to interpret using the above formulations (i) and (ii) of hypersurfaces; however, the third formulation on $T^{*} N \times \mathbb{R}$ is well-suited to this theorem. Consider the inclusion $\psi: \hat{\Sigma}_{N} \times \mathbb{R} \rightarrow \mathbb{J}^{1}(N, \mathbb{R})$. (Recall that ${ }^{\wedge}$ indicates the affine de-projectivization of a projective variety, resulting in a cone.) The eikonal system of $\Sigma_{N}$ is the exterior differential system $\mathscr{E}\left(\Sigma_{N}\right)=\psi^{*}(\mathcal{J})$ on $\hat{\Sigma}_{N} \times \mathbb{R}$; that is, $\mathscr{E}\left(\Sigma_{N}\right)$ is generated by $\psi^{*}(\Upsilon)$ and $\psi^{*}(\mathrm{~d} \Upsilon)$ and has independence condition $\mathrm{d} x^{1} \wedge \ldots \wedge \mathrm{d} x^{n} \neq 0$. An integral manifold of $\mathscr{E}\left(\Sigma_{N}\right)$ corresponds to a hypersurface in $N$ whose tangent space in annihilated by a section of $\hat{\Sigma}_{N}$.

We do not prove involutivity of $\mathcal{E}\left(\Sigma_{N}\right)$ in any significant case here; it is typically extremely deep and difficult, and references are provided below. However, the situation in Theorem 12.1 has several interesting consequences and interpretations.

12.2. Corollary. Suppose that the eikonal system of $\Sigma_{N}$ is involutive. Let $\ell-1$ denote the projective fiber dimension of $\Sigma_{N}$. The hypersurfaces guaranteed by Theorem 12.1 depend on $\ell$ functions of one variable.

Proof. Fix $[\varphi] \in\left(\Sigma_{N}\right)_{x}$. We work locally ${ }^{25}$ near $\varphi$, so we may assume $N$ is open, connected, and simply connected, and that $T^{*} N=N \times \mathbb{R}^{n}$. Because $\hat{\Sigma}_{N}$ is smooth with affine fiber dimension $\ell$ in $T^{*} N$, we may choose local coordinates $\left(q_{1}, \ldots, q_{n}\right)$ on each fiber of $T^{*} N$ near $\varphi$ such that $\hat{\Sigma}_{N}$ is defined by $q_{\ell+1}=\ldots=q_{n}=0$ near $\varphi$.

For each $\lambda=1, \ldots, \ell$, let $\sigma^{\lambda} \in\left(\Sigma_{N}\right)_{x}$ denote the lines of 1 -forms specified as

$$
\left(0, \ldots, 0, q_{\lambda}, 0, \ldots 0\right)
$$

nonzero in the $\lambda$ slot, in these coordinates. By Theorem 12.1 there is a local hypersurface $H_{\lambda} \subset N$ and a corresponding local function $x^{\lambda}$ such that $\mathrm{d} x^{\lambda} \sim \sigma^{\lambda}$. Complete $x^{1}, \ldots, x^{\ell}$ to a local coordinate system $\left(x^{i}\right)$ on $N$, and let $p_{i}$ be the canonical Darboux coordinates (that is, roughly corresponding to $\left.\frac{\partial y}{\partial x^{i}}\right)$ on the fiber of $T^{*} N$. Note that $p_{i}\left(\mathrm{~d} x^{\ell}\right)=\delta_{i}^{\lambda}$ by construction, so $\hat{\Sigma}_{N}$ is defined by $p_{\ell+1}=\ldots=p_{n}=0$. (Note that the open neighborhood of $T^{*} N$ around $\varphi$ may have shrunk during this process, which is why this is microlocal.)

\footnotetext{
${ }^{25}$ In fact, we work microlocally in the bundle. Microlocally means that we are working over a contractible neighborhood of the base space with a local trivialization of the bundle, and also within a neighborhood in the fiber.
} 
Therefore, the contact system on $T^{*} N \times \mathbb{R}$ is generated in a neighborhood of $\varphi$ by $\Upsilon=\mathrm{d} y-p_{i} \mathrm{~d} x^{i}$, which pulls back to $\hat{\Sigma}_{N} \times \mathbb{R}$ as

$$
\psi^{*}(\Upsilon)=\mathrm{d} y-p_{\lambda} \mathrm{d} x^{\lambda}
$$

The corresponding tableau is the space of $1 \times \ell$ matrices with entries $\mathrm{d} p_{\lambda}$ for $\lambda=1, \ldots, \ell$, so it has $s_{1}=s_{2}=\ldots=s_{\ell}=1$.

This is an interesting proof, using all three perspectives of hypersurfaces. The implicit function theorem on the fiber provides local coordinates on the base by involutivity. Then, the Frobenius theorem on the base produces contact coordinates on the fiber that are compatible with the original fiber coordinates. It is easy to adapt this proof to the following corollary, which is useful for constructing coordinates in some situations, as in Smi14].

12.3. Corollary. For any $\Sigma_{N}$, let $\left\langle\Sigma_{N}\right\rangle$ denote its linear span, which is itself a projective subbundle of $\mathbb{P} T^{*} N$. If $\mathscr{E}\left(\Sigma_{N}\right)$ is involutive, then $\mathscr{E}\left(\left\langle\Sigma_{N}\right\rangle\right)$ is involutive.

We will now examine several interpretations of the eikonal system that tie together various branches of geometry. Compare Sections $12(\mathrm{a}), 12(\mathrm{~b})$ and 13 to $\mathrm{BCG}^{+90}$. $\mathrm{V} \S 3(\mathrm{vi})]$.

12(a). As Lagrangian geometry. The $\mathbb{R}$ term in $T^{*} N \times \mathbb{R}$ plays little role for the eikonal system $\mathcal{E}\left(\Sigma_{N}\right)$. It is there merely to make obvious the relationship between the eikonal equations and hypersurfaces.

Instead, consider the symplectic manifold $T^{*} N$ with symplectic 2 -form $\mathrm{d} \Upsilon$, which is expressed in local coordinates as $\mathrm{d} \Upsilon=-\mathrm{d} p_{i} \wedge \mathrm{d} x^{i}$ according to Darboux's theorem. The Lagrangian Grassmannian $L G(N)$ is the bundle over $T^{*} N$ whose fiber is all the Legendrian $n$-planes

$$
L G_{\varphi}(N)=\left\{e \in \operatorname{Gr}_{n}\left(T_{\varphi} T^{*} N\right):\left.\mathrm{d} \Upsilon\right|_{e}=0\right\}, \quad \forall \varphi \in T^{*} N
$$

Each fiber is isomorphic to the homogeneous space $L G(n, 2 n)$, which is the variety of $n$-planes in $\mathbb{R}\left[x^{1}, \ldots, x^{n}, p_{1}, \ldots, p_{n}\right]$ on which $\mathrm{d} p_{i} \wedge \mathrm{d} x^{i}=0$. If we consider a plane $e \in$ $L G(n, 2 n)$ for which $\mathrm{d} x^{1} \wedge \ldots \wedge \mathrm{d} x^{n} \neq 0$, then $\mathrm{d} p_{i}=P_{i, j}(e) \mathrm{d} x^{i}$ on $e$ with $P_{i, j}=P_{j, i}$. Hence, the non-vertical open neighborhood of $L G(n, 2 n)$ is identified with the space of symmetric $n \times n$ matrices, $\operatorname{Sym}^{2}\left(\mathbb{R}^{n}\right)$.

Suppose the de-projectivized affine subvariety $\hat{\Sigma}_{N} \subset T^{*} N$ is defined smoothly by homogeneous functions in the local fiber variables $\left(p_{i}\right)$ of $T^{*} N$. From this perspective, the eikonal system $\mathcal{E}\left(\Sigma_{N}\right)$ is measuring the intersection of $\operatorname{Gr}_{n}\left(T_{\varphi} \Sigma_{N}\right)$ with $L G_{\varphi}(N)$ for all $\varphi \in \Sigma_{N}$.

12.5. COROLlary. The eikonal system $\mathcal{E}\left(\Sigma_{N}\right)$ is involutive if and only if there are local coordinates of $T^{*} N$ near $\varphi \in \hat{\Sigma}_{N}$ in which the non-vertical open set in $\operatorname{Gr}_{n}\left(T \Sigma_{N}\right) \cap L G(N)$ is described as the $n \times n$ symmetric matrices $P_{i, j}(e)$ that vanish outside the upper-left $\ell \times \ell$ part.

Proof. If the eikonal system $\mathscr{E}\left(\Sigma_{N}\right)$ is involutive, then we may construct coordinates as in Corollary 12.2 such that the de-projectivized affine variety $\hat{\Sigma}_{N}$ is defined by $p_{\varrho}=0$ 
for all $\varrho>\ell$, so $T_{\varphi} \hat{\Sigma}_{N}$ is defined by $\mathrm{d} p_{\varrho}=0$ for all $\varrho>\ell$. In such coordinates, the open neighborhood of the Lagrangian Grassmannian takes the block form

$$
\left.\left(\begin{array}{l}
\mathrm{d} p_{\lambda} \\
\mathrm{d} p_{\varrho}
\end{array}\right)\right|_{e}=\left.\left(\begin{array}{ll}
P_{\lambda, \mu}(e) & P_{\lambda, \varsigma}(e) \\
P_{\varrho, \mu}(e) & P_{\varrho, \varsigma}(e)
\end{array}\right)\left(\begin{array}{l}
\mathrm{d} x^{\mu} \\
\mathrm{d} x^{\varsigma}
\end{array}\right)\right|_{e}, \text { such that } P_{i, j}=P_{j, i},
$$

using our index convention 1.8 from Section 1 . The condition $e \in T \Sigma_{N}$ implies $\mathrm{d} p_{\varrho}=0$, so the lower blocks are zero. The matrix is symmetric, so the upper-right block is zero.

Conversely, suppose such coordinates exist. Then $T \hat{\Sigma}_{N}$ satisfies the closed 1-forms $\mathrm{d} p_{\varrho}=0$, and the dimensions match, so $\Sigma_{N}$ satisfies $p_{\varrho}=$ constant. Since the equations defining $\Sigma_{N}$ are homogeneous, it must be $p_{\varrho}=0$. Using these coordinates for $T^{*} N \times \mathbb{R}$ and $\mathcal{J}$ yields $\psi^{*}(\Upsilon)=\mathrm{d} y-p_{\lambda} \mathrm{d} x^{\lambda}$, as in Corollary 12.2 which is involutive with the correct Cartan characters and gives the desired hypersurfaces in Theorem 12.1 .

Compare this to Proposition 3.22 in $\left[\mathrm{BCG}^{+} 90\right.$, Chapter V]. For more symplectic and Lagrangian geometry, see Bry95.

12(b). As Poisson brackets. If $T^{*} N$ describes the state of a physical system, a function $F: T^{*} N \rightarrow \mathbb{R}$ is called an observable [SW86]. The Poisson bracket of observables is the operation given in local coordinates by

$$
\{F, G\}=\sum_{i}\left(\frac{\partial F}{\partial p_{i}} \frac{\partial G}{\partial x^{i}}-\frac{\partial G}{\partial p_{i}} \frac{\partial F}{\partial x^{i}}\right)=\sum_{i} \mathrm{~d} F \wedge \mathrm{d} G\left(\frac{\partial}{\partial p_{i}}, \frac{\partial}{\partial x^{i}}\right) .
$$

The Poisson bracket plays a fundamental role in Hamiltonian mechanics and the relationship between symmetries and conservation laws in physics. This is because (12.7) is a Lie bracket on $C^{\infty}\left(T^{*} N\right)$. (See Bry95 for details.)

Suppose that $O$ is some subspace of $C^{\infty}\left(T^{*} N\right)$, so that $O$ is a nonempty set of smooth observables that is closed under linear combinations. Suppose also that $\{F, G\} \in O$ for all $F, G \in O$. Then, $O$ is a Lie subalgebra of $C^{\infty}\left(T^{*} N\right)$ with respect to the Poisson bracket.

Because $\Sigma_{N} \subset \mathbb{P} T^{*} N$ is a projective variety in each fiber, the de-projectivized affine subvariety $\hat{\Sigma}_{N} \subset T^{*} N$ is defined smoothly by observables that take the form of homogeneous functions in the local fiber variables $\left(p_{i}\right)$ of $T^{*} N$. For convenience, let us make the additional assumption that the homogeneous functions are algebraic of degree $d$ in $\left(p_{i}\right)$, so that $\hat{\Sigma}_{N}$ is defined smoothly near $\varphi \in \hat{\Sigma}_{N}$ for $\varphi \neq 0$ by a set of equations in multi-index form

$$
0=F^{\varrho}(x, p)=\sum_{|I|=d} f^{\varrho, I}(x) p_{I}, \text { for } \varrho=\ell+1, \ldots, n .
$$

12.9. Corollary. Let $O$ denote the module in $S=C^{\infty}(N)\left[p_{1}, \ldots, p_{n}\right]$ generated by 12.8. The eikonal system $\mathcal{E}\left(\Sigma_{N}\right)$ is involutive if and only if $\{O, O\} \subset O$. That is, $\mathcal{E}\left(\Sigma_{N}\right)$ is involutive if and only if the module $O$ is a Lie algebra with respect to the Poisson bracket.

A proof - which does not depend on the polynomial form 12.8 - can be derived from Corollary 12.5 along with the observation that the Poisson bracket can be defined in a coordinate-free way as the operator such that

$$
\{F, G\}(\mathrm{d} \Upsilon)^{\wedge n}=n \mathrm{~d} F \wedge \mathrm{d} G \wedge(\mathrm{d} \Upsilon)^{\wedge(n-1)} .
$$


Equations of the form (12.8) appear in analysis as systems of homogeneous first-order PDEs on $u: \mathbb{R}^{n} \rightarrow \mathbb{R}$ of the form

$$
0=F^{\varrho}(x, u, \nabla u)=\sum_{|I|=d} f^{\varrho, I}(x) \frac{\partial u}{\partial x^{I}}, \text { for } \varrho=\ell+1, \ldots, n \text {. }
$$

A famous example is the $n-\ell=1$ characteristic equation for the wave equation of Section 6(c)

$$
0=-\left(u_{t}\right)^{2}+c^{2}\left(\left(u_{x}\right)^{2}+\left(u_{y}\right)^{2}\right) .
$$

This is generalized to any involutive EDS in Section 13

13. Involutivity of the characteristic variety. We would like to apply the entire discussion from Section 12 to the case where $\Sigma_{N}$ is a characteristic variety, but first we must establish that $\Xi$ is well-defined in $\mathbb{P} T^{*} N$.

Suppose that $\iota: N \rightarrow M$ is a connected integral manifold of an involutive exterior differential system $(M, \mathcal{I})$, and that $\iota^{(1)}(N)$ lies in $M^{(1)}$, a smooth and Kähler-regular component of $\operatorname{Var}_{n}(\mathcal{I})$, as in Section 4

Fix $x \in N$, and suppose $\iota(x)=p \in M$ and $\iota^{(1)}(x)=e \in M^{(1)}$. For $\xi \in \Xi_{e} \subset V_{e}^{*}$, we can consider the pullback $\iota^{(1) *}(\xi) \in \mathbb{P} T_{x}^{*} N \otimes \mathbb{C}$. In a basis $\left(\eta^{i}\right)$ of $T_{x}^{*} N$, we can write a representative as $\xi=\xi_{i} \eta^{i}$ for coefficients $\xi_{i} \in \mathbb{C}$. As a bundle over $N$, we have $\iota^{(1) *}(\xi)=\xi_{i} \eta^{i} \in \mathbb{P} T^{*} N \otimes \mathbb{C}=\gamma_{N}^{*}$. In this sense, we can pull back the characteristic variety - as a set- to $N$.

More precisely, recall that $\Xi$ has degree $s_{\ell}$ and affine fiber dimension $\ell$, but it is a scheme defined by the characteristic sheaf $\mathscr{M}$. For any local section $\left(u_{i}\right)$ of the coframe bundle $\mathcal{F}_{\boldsymbol{\gamma}^{*}} \rightarrow M^{(1)}$, we can write the characteristic sheaf $\mathscr{M}$ as a homogeneous ideal in the module $C^{\infty}\left(M^{(1)}\right)\left[u_{1}, \ldots, u_{n}\right]$. At each $e=\iota^{(1)}(x) \subset M^{(1)}$, the coframe $\left(u_{i}\right)$ is just a complex basis of $e$. Therefore, we obtain a basis for $T_{x} N$ of the form $\eta_{i}=\left(\iota_{*}^{(1)}\right)^{-1}\left(u_{i}\right)$. That is, in some neighborhood of $x$, the section $\left(\eta_{i}\right)$ of $\mathcal{F}^{*} N$ is well-defined. Moreover the stalks of the sheaf $C^{\infty}\left(M^{(1)}\right)$ can be pulled back, as $\iota^{(1) *}(f)$ is well-defined for any $f$ defined in a neighborhood of $e$. Therefore, we can pull back both the coefficients and the coordinates to define the homogeneous ideal $\mathscr{M}_{N}$ in $C^{\infty}(N)\left[\eta_{1}, \ldots, \eta_{n}\right]$. Let $\Xi_{N} \subset$ $\mathbb{P} T^{*} N \otimes \mathbb{C}$ be the scheme defined by $\mathscr{M}_{N}$.

Now, the entire discussion from Section 12 applies where $\Sigma_{N}$ is any particular component of $\Xi_{N}$. We focus our attention on the maximal smooth locus $\Xi_{N}^{o}$ of $\Xi_{N}$. We know additionally that $\Xi_{N}$ takes the polynomial form 12.8 as derived from (7.14), so it has degree $s_{\ell}$ and fiber dimension $\ell-1$ at smooth points, as a complex projective variety.

13.1. ThEOREM (Guillemin-Quillen-Sternberg). Suppose that $N$ is an ordinary integral manifold of an involutive exterior differential system $\mathcal{I}$ with character $\ell$ and Cartan integer $s_{\ell}$. The eikonal system of the smooth locus of the (complex) characteristic variety, $\mathscr{E}\left(\Xi_{N}^{o}\right)$, is involutive. At smooth points in $\Xi_{N}$, the characteristic hypersurfaces are parametrized by one function of $\ell$ variables.

Note that our definition of $\Xi_{N}$ is the complex characteristic variety ${ }^{26}$ Theorem 13.1

\footnotetext{
${ }^{26}$ Recall that, in the complex case, the distinction between elliptic and hyperbolic second-order PDEs does not occur, because there is only one nondegenerate signature.
} 
is called the "integrability of characteristics." Cartan demonstrated several examples of this phenomenon in Car11. The proof appears in GQS70, where a major step is the application of Theorem 7.6. Hence, this result appears to rely in an essential way on all three facets of the characteristic variety seen in Part III.

The converse of Theorem 13.1 is not true; it is easy to write down non-involutive exterior differential systems for which $\mathscr{E}\left(\Xi_{N}\right)$ is involutive.

However, in Gab81, Ofer Gabber proved a more general form of Theorem 13.1 that was conjectured in GQS70 and that removes practically all of the technical assumptions. Phrased as Theorem 13.2, Gabber's theorem recalls the ideas of Section 12(b).

13.2. THEOREM (Gabber). Let $S$ be a filtered ring whose graded ring $\operatorname{gr}(S)$ is a Noetherian commutative algebra over $\mathbb{Q}$. Let $M$ be a $\operatorname{gr}(S)$-ideal that is finitely generated as an $S$-module. Then $\{\sqrt{M}, \sqrt{M}\} \subset \sqrt{M}$.

In our context, Gabber's theorem applies to the case where $S=C^{\infty}(N)\left[p_{1}, \ldots, p_{n}\right]$, the ring of polynomials in local fiber variables of $T^{*} N$, filtered by degree. Then, $\operatorname{gr}(S)$ is the ring of homogeneous polynomials, graded by degree, which admits a Poisson structure like (12.7). The $\operatorname{gr}(S)$-ideal $M$ is the characteristic sheaf $\mathscr{M}_{N}$, which by 7.14 is defined by homogeneous polynomials if the original exterior differential system is involutive. By Hilbert's Nullstellensatz, the radical ideal $\sqrt{M}$ defines the generic component $\Xi_{N}^{o}$. Thus, the conclusion $\{\sqrt{M}, \sqrt{M}\} \subset \sqrt{M}$ invokes Corollary 12.9 to say that the eikonal system $\mathscr{E}\left(\Xi_{N}^{o}\right)$ is involutive.

From the general discussion of eikonal systems surrounding Theorem 12.1 , the interpretation of these theorems is apparent, in the form of Corollary 13.3 .

13.3. Corollary. Suppose that $N$ is an ordinary integral manifold of an involutive exterior differential system $\mathcal{I}$ with character $\ell$ and Cartan integer $s_{\ell}$. Then $N$ admits a local-possibly complex-coordinate system $\left(x^{1}, \ldots x^{n}\right)$ such that $\mathrm{d} x^{1}, \ldots, \mathrm{d} x^{\ell} \in \Xi_{N}$.

In [Smi14, the linear span of the characteristic variety, $\left\langle\Xi_{N}\right\rangle$ is studied in comparison to the Cauchy retraction space $\mathfrak{g}_{N}^{\perp}=\iota^{*}(\mathfrak{g})$, where $\mathfrak{g}^{\perp}$ is the maximum Frobenius system within $\mathcal{I}$, as in Section 5(b).

Suppose that the affine fiber dimension of $\left\langle\Xi_{N}\right\rangle$ is $L$ and that the affine fiber dimension of $\mathfrak{g}_{N}^{\perp}$ is $\nu$. These spaces are nested, so $\ell \leq L \leq \nu \leq n$.

13.4. Corollary. Suppose that $N$ is an ordinary integral manifold of an involutive exterior differential system $\mathcal{I}$ with character $\ell$ and Cartan integer $s_{\ell}$. Then $N$ admits a local-possibly complex-coordinate system $\left(x^{1}, \ldots, x^{n}\right)$ such that $\mathrm{d} x^{1}, \ldots, \mathrm{d} x^{\ell} \in \Xi_{N}$, such that $\mathrm{d} x^{\ell+1}, \ldots, \mathrm{d} x^{L} \in\left\langle\Xi_{N}\right\rangle$, and such that $\mathrm{d} x^{L+1}, \ldots, \mathrm{d} x^{\nu} \in \mathfrak{g}_{N}^{\perp}$.

Corollary 13.4 is a simple result, but its proof relies on building a coframe of $N$ in which the nilpotent parts of the commuting symbol maps $\mathrm{B}_{i}^{\lambda}$ are identified clearly; that is, it depends in an essential way on Theorems 13.1 and 5.4 The key point is that it reinforces the following remark.

13.5. REMARK (General dogma of the characteristic variety). An exterior differential system $(M, \mathcal{I})$ is a geometric object over $M$, meaning that its key properties are coordinateinvariant. On each Kähler-regular component $M^{(1)}$, knowing this geometry is equivalent to knowing the characteristic scheme and rank-1 variety over $M^{(1)}$, which are 
prolongation-invariant. Moreover, the geometry of an EDS imposes a geometry on its solutions, $\iota: N \rightarrow M$, and this imposition is also dictated by the characteristic scheme and rank-1 variety. Therefore, exterior differential systems can be classified up to coordinate equivalence as "parametrized families of manifolds $N$ with associated characteristic geometry."

Remark 13.5 is not a theorem; it is an attitude.

To make this remark robust for a general exterior differential system, the scheme separating $\operatorname{Var}_{n}(\mathcal{I})$ into its components $M^{(1)}$ — each component smooth with its own fixed Cartan characters over some subvariety of $M$-would have to be studied, and very little progress has been made at that level of abstraction. Nonetheless, whenever some property of PDEs is encountered, Remark 13.5 urges us to ask "is this property really invariant, or an artifact of my coordinates?" which is best answered by asking "can this property be reinterpreted using the characteristic scheme?" Sections 14 and 15 discuss progress of this type.

14. Yang's hyperbolicity criterion. One of the great frustrations of the CartanKähler theorem is that it relies on the Cauchy-Kowalevski theorem, so it applies only in the analytic category. One can see its dramatic failure in the smooth category in [Lew57. However, this frustration has been escaped in some special cases by exploiting the structure ${ }^{27}$ of $\Xi$. For example

(i) ODE systems. Suppose that $(M, \mathcal{I})$ is involutive over $C^{\infty}$ and that $\Xi=\emptyset$. Then $\ell=0$, so the tableau $A$ is the trivial (irrelevant) subspace of $W \otimes V^{*}$. The prolonged system $\mathcal{I}^{(1)}$ on $M^{(1)}$ is Frobenius, and $M^{(1)}$ is merely a copy of $M$ whose fiber is the unique element of an integrable distribution. That integrable distribution is the Cauchy retraction space $\mathfrak{g}$ of $\mathcal{I}$ as in Section 5(b) so it must have been that $\mathcal{I}=\mathfrak{g}^{\perp}$. The flow-box theorem foliates $M$ by solutions in the smooth category. (Actually, in the Lipschitz category, by standard ODE theory!) If $N$ is a leaf of this foliation, then removing Cauchy retractions on the original exterior differential system $(M, \mathcal{I})$ yields the exterior differential system $(N, 0)$.

(ii) Empty systems. Suppose that $(M, \mathcal{I})$ is involutive over $C^{\infty}$ and that $\Xi=V^{*}$ with $\left(s_{1}, s_{2}, \ldots, s_{n}\right)=(r, r, \ldots, r)$. Then, the tableau $A$ is the total space $W \otimes V^{*}$. Therefore, $M^{(1)}$ is an open domain in $\operatorname{Gr}_{n}(T M)$, so $\mathcal{I}=0$, and there is no condition whatsoever ${ }^{28}$ on integral manifolds $\iota: N \rightarrow M$; however, the prolongation $\iota^{(1)}: N \rightarrow M^{(1)}$ would have to satisfy the contact ideal, forcing some regularity on $N$. We studied this EDS in Section 2

A less trivial special case is presented in [Yan87, which is the subject of this section 29

\footnotetext{
${ }^{27}$ If we take the broadest possible interpretation of Remark 13.5 to heart, then any possible escape from analyticity ought to arise from the structure of $\Xi$. However, the reader is cautioned again that a dogma is not a theorem.

${ }^{28}$ The most extreme and amusing exploitations of the flexibility of $\operatorname{Gr}_{n}(T M)$ come from the homotopy principle Gro86, EM02].

${ }^{29}$ As it happens, the attempt to understand [Yan87] in the context of $\mathrm{BCG}^{+} 90$, Chapter VIII] was the inspiration for computing the details shown in [Smi15] and the entire approach of these notes.
} 
A tableau $A \subset W \otimes V^{*}$ is called determined if $s_{1}=s_{2}=\ldots=s_{n-1}=r$ and $s_{n}=0$. That is, $s=(n-1) r$, so $t=r$, and $H^{1}(A) \cong W$. Cartan's test shows that a determined tableau is always involutive, so we may assume that $A$ is written in endovolutive form as in Theorem 5.4. The only nontrivial symbol endomorphisms in 1.20 are $\mathrm{B}_{\lambda}^{\lambda}=I_{r \times r}$ and $\mathrm{B}_{n}^{\lambda}$ for $\lambda=1, \ldots, n-1$, like this:

$$
\left(\mathrm{B}_{i}^{\lambda}\right)=\left[\begin{array}{cccccc}
I_{r} & 0 & 0 & \ldots & 0 & \mathrm{~B}_{n}^{1} \\
& I_{r} & 0 & \ldots & 0 & \mathrm{~B}_{n}^{2} \\
& & \ddots & & \vdots & \vdots \\
& & & I_{r} & 0 & \mathrm{~B}_{n}^{n-2} \\
& & & & I_{r} & \mathrm{~B}_{n}^{n-1}
\end{array}\right] .
$$

The quadratic involutivity condition is trivial, which is why Cartan's test passes automatically.

14.2. Lemma. Suppose $A$ is determined and written in endovolutive bases. Identify $H^{1}(A)$ with $W$, and use our endovolutive basis of $W$ for both. Then for any $\varphi \in V^{*}$, the symbol map $\sigma_{\varphi}: w \mapsto \sigma(w \otimes \varphi)$ from Section $6(\mathrm{~b})$ is

$$
\sigma_{\varphi}=\left(\varphi_{\lambda} \mathrm{B}_{n}^{\lambda}-\varphi_{n} I\right)
$$

Then

$$
\operatorname{ker} \sigma_{\varphi}=\operatorname{ker}\left(\varphi_{\lambda} \mathrm{B}_{n}^{\lambda}-\varphi_{n} I\right)
$$

and the characteristic ideal $\mathscr{M}$ is generated by

$$
\operatorname{det} \sigma_{\varphi}=\operatorname{det}\left(\varphi_{\lambda} \mathrm{B}_{n}^{\lambda}-\varphi_{n} I\right) \text {. }
$$

In particular, $\xi \in \Xi$ if and only if $\xi_{n}$ is an eigenvalue of $\xi_{\lambda} \mathrm{B}_{n}^{\lambda}$.

Proof. The first two equations are immediate from our block form. From Part III] we know that $w \otimes \xi \in A$ if and only if $\mathrm{B}(\xi)(v) w=\xi(v) w$ for all $v$. Therefore, we compute in our endovolutive basis

$$
\begin{aligned}
\xi(v) w & =\mathrm{B}(\xi)(v) w=\xi_{\lambda} v^{i} \mathrm{~B}_{i}^{\lambda}(w) \\
& =\left(\xi_{\lambda} v^{\lambda}\right) w+\xi_{\lambda} v^{n} \mathrm{~B}_{n}^{\lambda} w=\left(\xi(v)-\xi_{n} v^{n}\right) w+\xi_{\lambda} v^{n} \mathrm{~B}_{n}^{\lambda} w .
\end{aligned}
$$

That is, $\xi_{n} w=\xi_{\lambda} \mathrm{B}_{n}^{\lambda} w$.

14.7. Corollary. Consider a determined tableau as in Lemma 14.2. Fix an integral element e. Suppose that $e^{\prime}$ is a real hyperplane in e such that $\left(e^{\prime}\right)^{\perp} \otimes \mathbb{C}=\varphi \in V^{*}$ and $\varphi \notin \Xi$. Then $\sigma_{\varphi}: W \rightarrow H^{1}(A)$ is an isomorphism.

Proof. By Lemma 14.2, we have $\operatorname{ker} \sigma_{\varphi} \neq 0$ if and only if $\varphi \in \Xi$.

14.8. Definition. Suppose $e^{\prime}$ is a real hyperplane in $e$ corresponding to the real covector $\varphi=\left(e^{\prime}\right)^{\perp} \in \mathbb{P} e^{*}$. The real hyperplane is called space-like if the following conditions hold.

(i) $\varphi \otimes \mathbb{C} \notin \Xi_{e}$.

(ii) For any $\eta \in \mathbb{P} e^{*}$, there is a real basis of $W$ in which $\left(\sigma_{\varphi}\right)^{-1}\left(\sigma_{\eta}\right): W \rightarrow W$ is real and diagonal.

(iii) The above choice of basis is a smooth function of $[\eta] \in e^{*} / \varphi=\left(e^{\prime}\right)^{*}$. 
A determined tableau $A \subset W \otimes V^{*}$ is called determined hyperbolic if $V$ admits a (real) space-like hyperplane.

Here is a simple example using our notation from Lemma 14.2 Fix $n=3$. To meet the first condition, suppose that $\varphi=1 u^{1}+0 u^{2}+0 u^{3}$ is not in $\Xi$. Then $\sigma_{\varphi}=\mathrm{B}_{3}^{1}$, and 0 is not an eigenvalue of $\sigma_{\varphi}$, which of course implies that $\sigma_{\varphi}=\mathrm{B}_{3}^{1}$ is invertible. Say $\eta=$ $0 u^{1}+1 u^{2}+\tau u^{3}$, so that $\sigma_{\eta}=\mathrm{B}_{3}^{2}-\tau I_{r}$. The second condition is that $\left(\mathrm{B}_{3}^{1}\right)^{-1}\left(\mathrm{~B}_{3}^{2}-\tau I_{r}\right)$ is diagonalizable using some change-of-basis $g_{\tau}$. The third condition is that $g_{\tau}$ is continuous in the projective variable $\tau$. Suppose moreover that we take our basis such that the basischange at $\tau=0$ is $g_{0}=I$. Then we have the condition that $\left(\mathrm{B}_{3}^{1}\right)^{-1} \mathrm{~B}_{3}^{2}$ is a diagonal matrix, $D$. This puts restrictions on the possible forms of these matrices. For example, $\operatorname{ker} \mathrm{B}_{3}^{2}=\operatorname{ker} D$ and $\operatorname{im} \mathrm{B}_{3}^{2} \subset \operatorname{im} \mathrm{B}_{3}^{1}$.

14.9. Definition. A tableau $A \subset W \otimes V^{*}$ is called hyperbolic if $V$ admits a flag given by a basis $\left(u^{1}, \ldots, u^{n}\right)$ of $V^{*}$ such that each of the sequential initial value problems from $\left\langle u^{i}, \ldots, u^{n}\right\rangle^{\perp}$ to $\left\langle u^{i+1}, \ldots, u^{n}\right\rangle^{\perp}$ has a hyperbolic determined tableau.

14.10. THEOREM (Yang). Theorem 5.3 applies in the smooth category, if A is hyperbolic.

The proof proceeds by replacing the Cauchy-Kowalevski initial-value problem with the Cauchy initial-value problem for determined first-order quasilinear hyperbolic PDEs. See [Yan87] and Appendix A of [Kam89] for more details.

Clearly the definition of hyperbolic depends on the geometry of $\Xi$ and the symbol maps $\mathrm{B}_{i}^{\lambda}$; however, to the author's knowledge no one has succeeded in writing down the explicit criteria on $\mathrm{B}_{i}^{\lambda}$ or $\mathscr{C}$ or $\Xi$ for general hyperbolicity. Hence, Yang's condition is not yet available to computer algebra systems. If that can be accomplished, it means we can identify a subvariety of the moduli of involutive tableaux - as in Section 5(a) that admit solutions in the smooth category.

One well-understood special case is when $\ell=1$, so $\Xi_{e}$ contains $s_{1}$ real points (with multiplicity). If the number of distinct points is sufficiently large (greater than $n$ ), then this is the situation for hyperbolic systems of conservation laws, as in [Tsa91]. The eikonal system is rigid, so each solution is foliated by $s_{1}$ characteristic hypersurfaces. Multiplicity corresponds to nilpotent pieces of the generalized eigenspaces of the symbol endomorphisms $B_{i}^{1}$. See again Section 8 .

15. Open problems and future directions. Our perspective here has been simpleminded - focusing on matrices and their computable properties - to gain intuition of $\Xi$ and $\mathscr{E}(\Xi)$ as rapidly as possible. The articles [Smi15] and [Smi14] are founded on this perspective, but reveal additional detail in the structures discussed here. For more modern and sophisticated treatment, please see [Mal03], KL07], and [CGG09]. Additionally, Chapters V-VIII of $\left[\mathrm{BCG}^{+} 90\right]$ contain significantly more results than we have summarized here.

To conclude, here are some interesting questions which-to the author's present knowledge - are open subjects that represent the major theoretical gaps in the subject of exterior differential systems. They are worth serious consideration as research projects, 
and offer great opportunities for collaboration between analysts, differential geometers, algebraic geometers, and scientific programmers.

(i) Variety of involutive tableaux. For given $r, n$, and Cartan characters $\left(s_{1}, \ldots, s_{\ell}\right)$, what is the variety of involutive tableaux (with fixed coefficients)? Can we compute its dimension or degree or Hilbert polynomial? Section 5(a) demonstrates a first step toward understanding the variety of involutive tableaux, as Theorem 5.4 gives the ideal in certain bases. However, to answer the question completely, one would need to examine how the coefficients in (5.6) vary under arbitrary changes of basis in $V^{*}$ and $W$.

(ii) Special hyperbolic integrability criteria. Solution techniques (such as Lax pairs, inverse scattering, hydrodynamic reduction, and Bäcklund transformations) play a key rôle in the analysis of wave-like PDEs, especially those coming from physics and geometry. Given that these techniques are coordinate-invariant, Remark 13.5 suggests that they should all be expressible as algebraic conditions on $\mathscr{M}$. Expressing those conditions in an abstract way over $\Xi$ and $M^{(1)}$ would allow more systematic geometric approach to many of the ad hoc methods in the analysis of PDEs 30

(iii) Elliptic systems. Consider the classical results regarding elliptic regularity of quasilinear elliptic operators. This is another form of "special integrability criteria." How far can the notion of elliptic regularity be extended to general exterior differential systems? Certainly the conditions of involutivity, $\langle\Xi\rangle=V^{*}$, and $\Xi_{\mathbb{R}}=\emptyset$ are necessary, and one can directly translate the classical theorems to an EDS written specifically to describe a quasilinear second-order elliptic operator in local coordinates, but what other technical assumptions can be dropped? Some discussion appears in $\left[\mathrm{BCG}^{+} 90\right.$, Chapter X§3].

(iv) Moduli of involutive tableaux. Refining the first problem in light of the second and third problems, can we identify invariant sub-varieties of the variety of involutive tableaux? Dogma 13.5 indicates that we should be able to identify subvarieties, such as hyperbolic tableaux, elliptic tableaux, systems satisfying special integrability conditions, and so on. What does it mean when these sub-varieties intersect? Lewy showed that there are involutive PDEs with no solution in the smooth category [Lew57, which cannot happen in the analytic category. Where do the Lewy examples fall in this variety? Are there other subvarieties that have not been observed in classical equations? If there is any organizing geometry behind the "nearly impenetrable jungle" of involutive PDEs, this is where we should look.

(v) Weakness of involutivity of characteristics. Note that Theorem 13.2 does not regard the involutivity of an exterior differential system in any direct way; the assumption of involutivity of $\mathcal{I}$ enters Theorem 13.2 only because we know that $\mathscr{M}_{N}$ is an ideal of homogeneous polynomials from (7.14). Thus, we expect that the condition $" \Xi_{N}$ is the characteristic scheme of an exterior differential system $\mathcal{I}$, and $\mathscr{E}\left(\Xi_{N}\right)$

\footnotetext{
${ }^{30}$ Indeed, the central theme of the conference for which these notes were prepared was to express Ferapontov's notion of hydrodynamic integrability in terms of algebro-geometric structures in the Lagrangian Grassmannian. The notion of hydrodynamic integrability is tied completely to the secant variety of $\mathscr{C}$.
} 
is involutive" is much weaker than " $\Xi_{N}$ is the characteristic scheme of an exterior differential system $\mathcal{I}$, and $\mathcal{I}$ is involutive." The gap between these two statements is extremely important to explore, as it goes to the heart of the question about how involutivity leads to solutions of the initial-value problem for a system of PDEs. To put this a different way, can we construct an embedded variety $\Xi_{N} \subset \mathbb{P} T^{*} N$ that is involutive, but for which there is no involutive exterior differential system for which $\Xi$ is the characteristic variety?

(vi) Global integrability of the characteristic variety. If $A$ is involutive, then the system $\mathscr{E}\left(\Xi_{N}^{o}\right)$ is involutive on an ordinary integral manifold, $N$. However, it is not clear whether $\Xi^{o}$ is involutive as a bundle over $M^{(1)}$ itself in any reasonable way that considers all $N$ simultaneously. That is, consider the EDS on $M^{(1)}$ generated by $\mathcal{I}^{(1)}+\langle\xi\rangle$ for some section $\xi$ of $\Xi^{o} \subset V^{*} \subset \mathbb{P} T M^{(1)} \otimes \mathbb{C}$. Under what circumstances is this involutive? Can Gabber's theorem 13.2 be adopted to this case? This has theoretical implications for special integrability conditions (above), because it would allow one to count special solutions among all solutions from $M^{(1)}$ directly. Additionally, given its algebraic nature, can Gabber's theorem provide solutions for certain types of PDEs with low regularity, bypassing the Lewy examples with various additional conditions?

(vii) Prolongation theorems. Does prolongation always uncover solutions of an exterior differential system, if we remove the regularity assumptions on $M^{(1)}$ and consider the many components of the scheme $\operatorname{Var}_{n}\left(\operatorname{Var}_{n}(\cdots(\mathcal{I}) \cdots)\right)$ ? As experts are well aware, this is has been the key open question in the subject for most of a century. (See $\left[\mathrm{BCG}^{+} 90\right.$, Chapter VI].) In the context of this monograph, the question is related to whether the block form of involutive tableau 1.20 and the involutivity conditions of Theorem 5.4 can be extended from $M^{(1)}$ to non-smooth points in $\operatorname{Var}_{n}(\mathcal{I})$ ? Because of the interaction of Guillemin normal form and involutivity with Spencer cohomology as in Section 9 , such an extension of the endovolutive block form could be helpful in an effort to construct (or prove the non-existence of) counterexamples.

(viii) Representation theory of Lie pseudogroups. Lie pseudogroups are subgroups of the diffeomorphism pseudogroup whose trajectories are the solutions of involutive PDEs. See Olv09. Just as Jordan form (in the guise of the Levi decomposition) is the key first step toward understanding the representation of Lie groups, it is reasonable to expect that the endovolutive block-form 1.20 and Theorem 5.4 can serve as the foundation of a representation theory of Lie pseudogroups. Any results regarding the "moduli of involutive tableaux" can be applied to Lie pseudogroups with those tableaux. Indeed, the first application of Theorem 13.1 was the classification of the primitive Lie pseudogroups GQS66.

Acknowledgments. Thanks to Robert L. Bryant, Niky Kamran, and Deane Yang for always humoring my interest in these details, to Ian Morrison for helping me appreciate the role of incidence correspondences in algebraic geometry, and to Giovanni Moreno for arranging this course and for encouraging me repeatedly to complete these notes. The punny photograph in Fig. 5 is public domain from the U.S. Fish and Wildlife Service. 


\section{References}

[Art91] M. Artin, Algebra, Prentice Hall, Englewood Cliffs, NJ, 1991.

[Bry95] R. L. Bryant, An Introduction to Lie Groups and Symplectic Geometry, IAS/Park City Math. Ser. 1, Amer. Math. Soc., Providence, RI, 1995.

$\left[\mathrm{BCG}^{+} 90\right]$ R. L. Bryant, S. S. Chern, R. B. Gardner, H. L. Goldschmidt, P. A. Griffiths, Exterior Differential Systems, Math. Sci. Res. Inst. Publ. 18, Springer, New York, 1991.

[CGG09] J. Carlson, M. Green, P. Griffiths, Variations of Hodge structure considered as an exterior differential system: old and new results, SIGMA Symmetry Integrability Geom. Methods Appl. 5 (2009), paper 087.

[Car11] É. Cartan, Sur les systèmes en involution d'équations aux dérivées partielles du second ordre à une fonction inconnue de trois variables indépendantes, Bull. Soc. Math. France 39 (1911), 352-443.

[Cle17] J. N. Clelland, From Frenet to Cartan: The Method of Moving Frames, Grad. Texts Math. 178, Amer. Math. Soc., Providence, RI, 2017.

[Eis05] D. Eisenbud, The geometry of syzygies, Grad. Texts in Math. 229, Springer, New York, 2005.

[EM02] Y. Eliashberg, N. Mishachev, Introduction to the h-Principle, Grad. Stud. Math. 48, Amer. Math. Soc., Providence, RI, 2002.

[FHK09] E. V. Ferapontov, L. Hadjikos, K. R. Khusnutdinova, Integrable equations of the dispersionless Hirota type and hypersurfaces in the Lagrangian Grassmannian, Int. Math. Res. Not. IMRN 2010, 496-535.

[Gab81] O. Gabber, The integrability of the characteristic variety, Amer. J. Math. 103 (1981), 445-468.

[Gar67] R. B. Gardner, Invariants of Pfaffian systems, Trans. Amer. Math. Soc. 126 (1967), 514-533.

[Gar89] R. B. Gardner, The Method of Equivalence and its Applications, CBMS-NSF Regional Conf. Ser. in Appl. Math. 58, SIAM, Philadelphia, 1989.

[Ger61] M. Gerstenhaber, On dominance and varieties of commuting matrices, Ann. of Math. (2) 73 (1961), 324-348.

[Gol67] H. L. Goldschmidt, Existence theorems for analytic linear partial differential equations, Ann. of Math. (2) 86 (1967), 246-270.

[Gro86] M. Gromov, Partial Differential Relations, Ergeb. Math. Grenzgeb. (3) 9, Springer, Berlin, 1986.

[Gui68] V. Guillemin, Some algebraic results concerning the characteristics of overdetermined partial differential equations, Amer. J. Math. 90 (1968), 270-284.

[GK68] V. Guillemin, M. Kuranishi, Some algebraic results concerning involutive subspaces, Amer. J. Math. 90 (1968), 1307-1320.

[GQS66] V. Guillemin, D. Quillen, S. Sternberg, The classification of the complex primitive infinite pseudogroups, Proc. Nat. Acad. Sci. U.S.A. 55 (1966), 687-690.

[GQS70] V. W. Guillemin, D. Quillen, S. Sternberg, The integrability of characteristics, Comm. Pure Appl. Math. 23 (1970), 39-77.

[GS00] R. M. Guralnick, B. Sethuraman, Commuting pairs and triples of matrices and related varieties, Linear Algebra Appl. 310 (2000), 139-148.

[Har92] J. Harris, Algebraic Geometry: A First Course, Grad. Texts in Math. 133, Springer, Berlin, 1992. 
[Har13] J. Harris, Parameter spaces in algebraic geometry, in: Samuel Eilenberg Lecture Series, Columbia University, New York, NY, 2013, https://www.youtube.com/watch? $\mathrm{v}=\_\mathrm{m}-\mathrm{cWF} 3 \mathrm{DY} 9 \mathrm{w} \&$ list=PLj6jTBBj-5B_QE35IEQgLkkEct0Dk8GG6

[IL03] T. A. Ivey, J. M. Landsberg, Cartan for Beginners: Differential Geometry via Moving Frames and Exterior Differential Systems, Grad. Stud. Math. 61, Amer. Math. Soc., Providence, RI, 2003.

[Kam89] N. Kamran, Contributions to the study of the equivalence problem of Élie Cartan and its applications to partial and ordinary differential equations, Acad. Roy. Belg. Cl. Sci. Mém. Collect. $8^{\circ}$ (2) 45 (1989), no. 7, 122 pp.

[KN63] S. Kobayashi, K. Nomizu, Foundations of Differential Geometry, vol. I, Interscience, New York, 1963.

[KL07] B. Kruglikov, V. Lychagin, Spencer $\delta$-cohomology, restrictions, characteristics and involutive symbolic PDEs, Acta Appl. Math. 95 (2007), 31-50.

[Lew57] H. Lewy, An example of a smooth linear partial differential equation without solution, Ann. of Math. (2) 66 (1957), 155-158.

[Mal03] B. Malgrange, Cartan involutiveness = Mumford regularity, in: Commutative Algebra (Grenoble/Lyon, 2001), Contemp. Math. 331, Amer. Math. Soc., Providence, RI, 2003, 193-205.

[McK18] B. McKay, Introduction to exterior differential systems, this volume, 45-55.

[Mil97] J. Milnor, Topology from the Differentiable Viewpoint, revised ed., Princeton Landmarks Math., Princeton Univ. Press, Princeton, NJ, 1997.

[MS74] J. W. Milnor, J. D. Stasheff, Characteristic Classes. Annals of Math. Stud. 76, Princeton Univ. Press, Princeton, 1974.

[Olv95] P. J. Olver, Equivalence, Invariants, and Symmetry, Cambridge Univ. Press, Cambridge, 1995.

[Olv09] P. J. Olver, J. Pohjanpelto, F. Valiquette, On the structure of Lie pseudo-groups, SIGMA Symmetry Integrability Geom. Methods Appl. 5 (2009), paper 077.

[Qui64] D. G. Quillen, Formal properties of over-determined systems of partial differential equations, Ph.D. thesis, Harvard University, 1964.

[Rob14] C. Robles, Schubert varieties as variations of Hodge structure, Selecta Math. (N.S.) 20 (2014), 719-768.

[SW86] D. H. Sattinger, O. L. Weaver, Lie Groups and Algebras with Applications to Physics, Geometry, and Mechanics, Appl. Math. Sci. 61, Springer, New York, 1986.

[Sha94] I. R. Shafarevich, Basic Algebraic Geometry, vol. 1, second ed., Springer, Berlin, 1994.

[SS65] I. Singer, S. Sternberg, The infinite groups of Lie and Cartan. I. The transitive groups, J. Anal. Math. 15 (1965), 1-114.

[Smi09] A. D. Smith, Integrable GL(2) geometry and hydrodynamic partial differential equations, Comm. Anal. Geom. 18 (2010), 743-790.

[Smi14] A. D. Smith, Degeneracy of the characteristic variety, arXiv:1410.6947

[Smi15] A. D. Smith, Constructing involutive tableaux with Guillemin normal form, SIGMA Symmetry Integrability Geom. Methods Appl. 11 (2015), paper 053.

[Spe62] D. C. Spencer, Deformation of structures on manifolds defined by transitive, continuous pseudogroups. II. Deformations of structure, Ann. of Math. (2) 76 (1962), 399-445.

[TB97] L. N. Trefethen, D. Bau III, Numerical Linear Algebra, SIAM, Philadelphia, 1997. 
[Tsa91] S. P. Tsarëv, The geometry of Hamiltonian systems of hydrodynamic type. The generalized hodograph method, Izv. Akad. Nauk SSSR Ser. Mat. 54 (1990), 1048-1068; English transl.: Math. USSR-Izv. 37 (1991), 397-419.

[Val13] F. Valiquette, Solving local equivalence problems with the equivariant moving frame method, SIGMA Symmetry Integrability Geom. Methods Appl. 9 (2013).

[Yan87] D. Yang, Involutive hyperbolic differential systems, Mem. Amer. Math. Soc. 68 (1987), no. 370. 